\title{
Ambulatory Gait Analysis : clinical application and fall risk detection
}

Citation for published version (APA):

Senden, R. H. J. (2013). Ambulatory Gait Analysis : clinical application and fall risk detection. [Doctoral Thesis, Maastricht University]. Datawyse / Universitaire Pers Maastricht.

https://doi.org/10.26481/dis.20130529rs

Document status and date:

Published: 01/01/2013

DOI:

10.26481/dis.20130529rs

Document Version:

Publisher's PDF, also known as Version of record

\section{Please check the document version of this publication:}

- A submitted manuscript is the version of the article upon submission and before peer-review. There can be important differences between the submitted version and the official published version of record.

People interested in the research are advised to contact the author for the final version of the publication, or visit the DOI to the publisher's website.

- The final author version and the galley proof are versions of the publication after peer review.

- The final published version features the final layout of the paper including the volume, issue and page numbers.

Link to publication

\footnotetext{
General rights rights.

- You may freely distribute the URL identifying the publication in the public portal. please follow below link for the End User Agreement:

www.umlib.nl/taverne-license

Take down policy

If you believe that this document breaches copyright please contact us at:

repository@maastrichtuniversity.nl

providing details and we will investigate your claim.
}

Copyright and moral rights for the publications made accessible in the public portal are retained by the authors and/or other copyright owners and it is a condition of accessing publications that users recognise and abide by the legal requirements associated with these

- Users may download and print one copy of any publication from the public portal for the purpose of private study or research.

- You may not further distribute the material or use it for any profit-making activity or commercial gain

If the publication is distributed under the terms of Article $25 \mathrm{fa}$ of the Dutch Copyright Act, indicated by the "Taverne" license above, 


\section{AMBULATORY GAIT ANALYSIS \\ Clinical application and fall risk detection}

Rachel Senden 
The research presented in this thesis was performed at the Department of Human Movement Sciences at Maastricht University and the Atrium Medical Center Heerlen, AHORSE foundation, Department Orthopaedic Surgery \& Traumatology.

Cover design: $\quad$ Nadine de Burlett

Printed by: $\quad$ Datawyse / Universitaire Pers Maastricht

(C) Copyright Rachel Senden, Maastricht 2013

ISBN: $\quad 9789461591821$

Publication of this thesis was financially supported by the Maatschap Orthopaedie Atrium MC, Faculty of Health, Medicine and Life Sciences Maastricht University, NUTRIM, ANNA foundation/NOREF, Maastricht Instruments \& IDEE, Servier Nederland Farma B.V., Synthes B.V., Biomet Nederland B.V., Dutch Arthritis Foundation, Atrium MC Leerhuis, Stöpler Orthopedie, Sports Medicine \& Combined Quality Care and Defauwes.
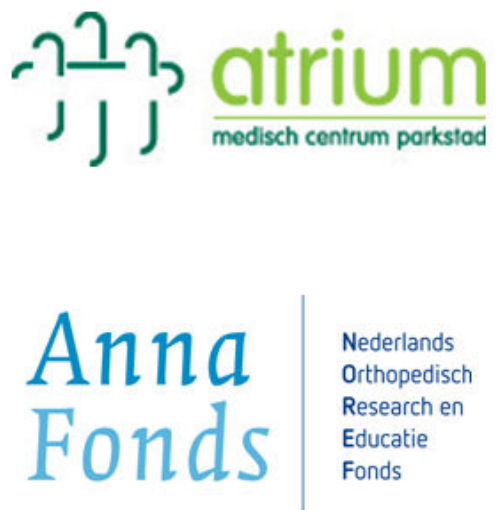

Nederlands Orthopedisch Research en Educatie Fonds
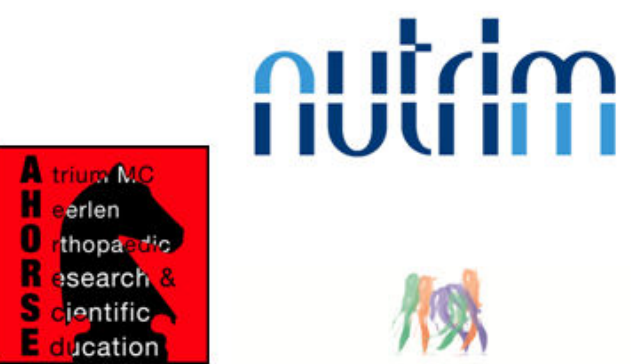

DEFAUWES

Beweeg vrijer 〈> Leef beter 


\title{
Ambulatory Gait Analysis \\ Clinical application and fall risk detection
}

\author{
PROEFSCHRIFT \\ ter verkrijging van de graad van doctor aan de Universiteit Maastricht, \\ op gezag van de Rector Magnificus, Prof. dr. L.L.G. Soete, \\ volgens het besluit van het College van Decanen, \\ in het openbaar te verdedigen \\ op woensdag 29 mei 2013 om 14 uur \\ door
}

Rachel Henriette Joseph Senden

Geboren te Tongeren op 22 januari 1983

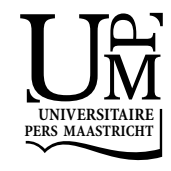




\section{PROMOTOR}

Prof. dr. I.C. Heyligers

\section{COPROMOTORES}

Dr. K. Meijer

Dr. B. Grimm

Dr. H.H.C.M. Savelberg

\section{BEOORDELINGSCOMMISSIE}

Prof. dr. M.K.C. Hesselink (voorzitter)

Prof. dr. R. de Bie

Prof. dr. A. Blom (Bristol University)

Dr. M. Pijnappels (Vrije Universiteit Amsterdam)

Prof. dr. R.J.E.M. Smeets 


\section{CONTENTS}

Chapter 1 General introduction

Chapter 2 Acceleration-based gait test for healthy subjects: Reliability and reference data

Chapter 3 The importance of correcting for individual differences in the 31 clinical diagnosis of gait disorders

Chapter 4 Acceleration based motion analysis as a tool for rehabilitation: Exploration in simulated functional knee limited walking conditions

Chapter 5 The importance to include objective functional outcomes in 53 the clinical follow up of total knee arthroplasty patients

Chapter 6 Accelerometry-based gait analysis, an additional objective 69 approach to screen subjects at risk for falling

Chapter 7 The 'TRiP', a specially designed experimental set-up to induce perturbations in a standardized way: A technical note

Chapter 8 The influence of age, muscle strength and speed of information processing on recovery responses to external perturbations in gait

Chapter 9 General discussion

Summary

Samenvatting

Dankwoord

List of publications

About the author 

Chapter 1

General introduction 
Gait, which is the scientific term for what individuals refer to as walking, is one of the most important activities of daily life for humans. It is a very economical means of locomotion. In clinical and research settings, gait performance is widely accepted as a general measure of functional ability among individuals. In addition, gait is associated with health, well-being and quality of life Studenski et. al. has shown that walking speed is related to survival rate in the elderly, and that its predictive power equals that of more regularly used metrics such as BMI and smoking (1). Dumurgier et. al. has indicated that a slow walking speed in older people is strongly associated with an increased risk of cardiovascular mortality (2). Annegarn et. al. has revealed that walking is the primary activity reported as problematic by patients with Chronic Obstructive Pulmonary Disease (COPD) (3). All this attests to the clinical relevance of human gait analysis.

Gait is not invariable, and can change due to several factors. Our walking pattern changes with advancing age and is furthermore influenced by body characteristics like sex, body height and mass (4-5). It is, for instance, well known that tall subjects take longer steps than small subjects and that men walk faster than women. Gait also changes as a result of health-related factors such as pain and diseases. For instance, individuals with pain in a lower extremity relieve the painful leg during walking, causing an asymmetric gait pattern, and elderly people with an increased fall risk adopt a conservative, cautious gait (e.g. small step length and wide step width) in order to increase stability (6). Moreover, the gait pattern can change through rehabilitation and training. A six months multitask exercise program for elderly people at risk of falling, for instance, reduced stride variability and thus contributes to a more stable gait pattern (7). These findings suggest that human gait analysis is useful for clinical purposes, for example to indicate symptoms, to monitor patients or to evaluate rehabilitation programs. However, to apply human gait analysis in clinical practice it is necessary for critical changes in human gait to be monitored in an accurate and reliable way.

Several approaches can be used to analyse gait. The gait pattern is a cyclic process consisting of repetitive gait cycles, indicating that relevant information can be captured from one complete gait cycle. A gait cycle is equivalent to a stride and describes the motion from initial foot contact (heel strike) with the stance phase, proceeding through a swing phase and ending with the limb's next initial contact. Comparing several individual strides allows the determination of gait variability (difference between gait cycles, strides) and asymmetry (differences in left and right leg movement, steps), which are considered important parameters in evaluating pathological gait (8-9). In laboratory settings, advanced motion capture systems like force plates and 3D optical motion capture systems allow the derivation of spatio-temporal (concerning stride analysis variables e.g. cadence, stride length), kinematic (concerning joint movements, e.g. joint angle) and kinetic (concerning the forces that produce the movement, e.g. muscle activity) gait 
parameters from a few steps. Although these motion capture systems provide an accurate, reliable and objective evaluation of gait (10), they are impractical for large subject groups as they are laboratory based, time consuming, complex and expensive. These systems are therefore mainly used for research purposes. In clinical practice gait analysis often relies on standard clinical scales, which include aspects of gait assessed by visual gait observation. For instance, the Knee Society Score assesses function in knee patients based on walking distance and walking aids, and the Tinetti scale assesses mobility in individuals based on observing, for instance, left-right leg asymmetry. These clinical scales are easy, non-invasive, relatively fast and cheap and therefore practical for clinical use. However they are also characterized by subjectivity and pain dominance, and they suffer a ceiling effect. Moreover, visually observing gait has proved to be inadequate for accurate assessment (11). This leads to a dispute about the validity, reliability and responsiveness of the clinical scales, and highlights the need for new clinical functional assessment tools able to evaluate function in individuals more objectively and precisely in a simple, systematic and controlled manner.

Following advances in sensor development during recent decades, accelerometers (fig.1) have been indicated as potential gait analysis systems for clinical use to assist or improve functional assessment (12-15). Accelerometers are objective and not dominated by pain. These sensors are non-invasive, relatively cheap, portable and do not require a specialized environment, allowing their application in clinical practice. Previous studies have investigated the use of accelerometers to analyse human gait. The reliability of accelerometers in identifying subsequent gait cycles has been examined $(12-13,16-18)$. The acceleration pattern of walking has been studied by relating specific peaks in the acceleration signal to specific phases in the gait cycle (e.g. heel strike, fig. 1) $(9,14,16,18-24)$ and studies have focused on acceleration-derived gait parameters such as spatio-temporal (e.g. walking speed $(9,13-14,18-20,22-26))$, variability and stability-related parameters (e.g. variability in gait rhythm, root mean square) $(9,13-14,20,26)$. These studies suggest that accelerometers are able to describe the gait pattern of individuals. However, these studies differ from each other regarding the set-up and the approach used. For instance, different methods of deriving gait parameters from trunk accelerations have been applied such as autocorrelations $(12-13,20,25)$ or peak detection methods $(14,19,23-24,26)$. Also, different fixation locations have been used such as the head $(9,21,26)$, the lower back $(9,12-14,18-21,23,25-29)$, the leg (24), the upper chest (22) and the forefoot (22). Fixation at the level of the lower lumbar spine and the sacrum, however, have proved to be the most reliable. This position is near to the centre of mass of the body, a region where the opposing rotations of the pelvis and thorax are most effectively neutralized $(9,12-13,16,19,23)$. In addition, most of the studies have been limited to small groups (range 22-26 subjects $(16,18,21-23,25,27,29))$ and specific populations (e.g. healthy subjects 
$(12,21,23,30)$, patients with Parkinson's disease (29), hemiparetic stroke (22), unilateral hip osteoarthritis (24) and dyslexia (25)). An exception is the study by Menz et. al. (26) in which gait of 100 community-dwelling elderly subjects is examined. Moreover, different accelerometer configurations have been used in previous studies: a single accelerometer (12-13, 16-18, 20, 23, 25-26) vs. multiple accelerometers and uni-, bi- or triaxial accelerometers $(14,22,24,27,31)$. A single triaxial accelerometer is a simple accelerometer configuration consisting of one sensor measuring accelerations in three degrees of freedom. A single sensor is small, lightweight, easy and fast to use and can easily be attached to, and detached from, a body segment. These features make a single accelerometer very attractive for clinical use.

All these findings together: the beneficial advantages of accelerometers, the promising results on acceleration-based gait analysis, the sacrum as the most reliable fixation place and the user-comfort of a single triaxial accelerometer suggest the clinical feasibility and practicality of a single triaxial accelerometer fixed at the level of the sacrum for describing gait of individuals. While many gait analysis studies have been performed using different acceleration-based approaches, no study has investigated the use of a single triaxial accelerometer measuring accelerations at the level of the sacrum, which will from now on be referred to as acceleration-based gait analysis (AGA), to describe gait of different population groups.

The application of AGA in clinical practice may be useful for several patient groups in different departments. For instance, in the orthopaedic department where many patients suffer problems at the lower extremity, in the neurological department where the functional ability of patients is affected by diseases such as cerebral palsy or Parkinson's disease, in the rheumatology department where patients suffer joint pain which influences their mobility, and in nursing homes where the elderly are screened for fall risk which is frequently based on mobility assessments. However, to use AGA for the assessment of human gait on various subject groups in clinical practice and in future studies a thorough evaluation and validation of its performance in different populations and settings is needed. In addition, a gait database of healthy subjects with varying subject characteristics (e.g. age, gender) is required for reference. This reference database ensures that gait is correctly interpreted and enables the detection of subtle changes in gait.

Since a cautious gait pattern has been associated with increased fall risk, AGA may be a useful additional tool for objectively assessing fall risk in the elderly population (26, 32-33). However, fall risk has been associated with many other risk factors, intrinsic (e.g. age, fall history) and extrinsic (e.g. trips and slips). To understand better why people fall and why individuals are at risk of falling, it is important to go beyond the identification of risk factors and investigate the causal factors of a fall in more detail. Since perturbations (e.g. trips) in gait have been identified as 


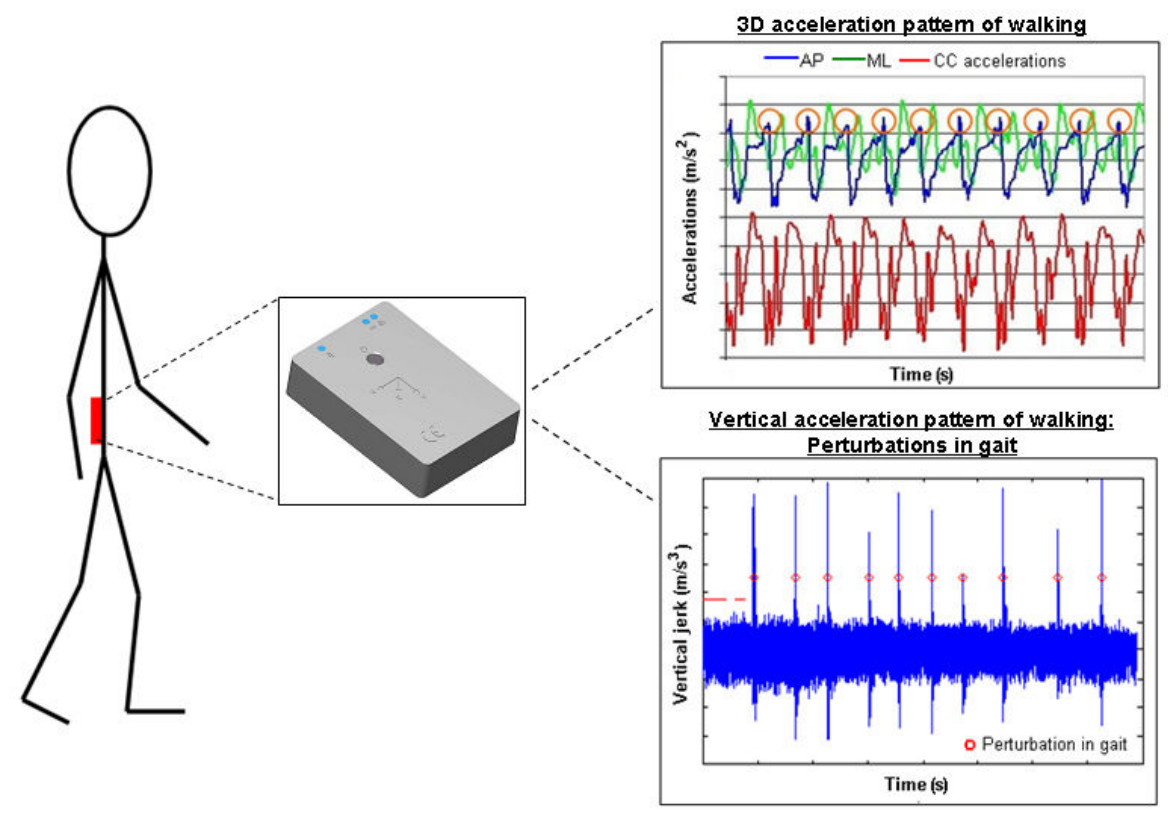

Fig. 1. The Triaxial accelerometer measuring trunk accelerations in anterior-posterior (AP), medio-lateral $(\mathrm{ML})$ and cranial-caudal $(\mathrm{CC})$ direction. The upper figure shows the 3D acceleration pattern of walking, showing a repeatable pattern for every step (o corresponds to heel strike). The lower figure shows the vertical jerk of the gait pattern, with the exceeding peaks representing external perturbations.

relevant markers for fall risk accounting for approximately $60 \%$ of the falls in elderly people (34-35), the last part of this thesis focuses on recovery responses to external perturbations in gait. Previous studies have identified two stereotypical recovery strategies (elevating vs. lowering strategy), which are employed by young and older subjects to compensate for balance disturbances during walking induced by external perturbations in gait (36). Interestingly, similar perturbation conditions yield different recovery responses in young and older subjects (37). In addition it is known that an (in)adequate recovery is influenced by physiological factors like muscle strength and reaction time (37-39). More insight into the factors modifying the recovery response that individuals employ when they encounter a perturbation in gait, is required to understand better why individuals fall and why they are at risk of falling. This insight may provide targets for fall prevention programs aimed at improving trip recovery and reducing fall incidence. 


\section{AIM AND OUTLINE OF THESIS}

The aim of this thesis is to evaluate the suitability of an acceleration-based gait analysis system that relies on a single triaxial accelerometer measuring accelerations at the level of the sacrum (AGA) for describing gait of various subject groups such as healthy subjects, orthopaedic patients and elderly people at risk of falling. To achieve this aim, three research questions are examined:

1. Can acceleration-based gait analysis be used to create a reliable reference gait database for healthy subjects? (chapters 2-3)

2. Is acceleration-based gait analysis of clinical use for patients with functional limitations in the lower extremities? (chapters 4-5).

3. Are gait parameters measured by acceleration-based gait analysis and the recovery responses to external perturbations in gait associated with fall risk? (chapters 6-8)

Chapters 2 and 3 investigate the first research question "Can acceleration-based gait analysis be used to create a reliable reference gait database for healthy subjects?". In Chapter 2, an acceleration-based reference gait database has been created. This database relies on a large sample group of healthy subjects with various characteristics (e.g. age and gender). The reliability of AGA is investigated, together with the sensitivity of AGA to subject characteristics. Chapter 3 explores and quantifies the effects of variations in age, gender, body height and body mass on various AGA-parameters. This information is required to interpret gait correctly. The second research question "Is acceleration-based gait analysis of clinical use for patients with functional limitations in the lower extremities?" is evaluated in chapters 4 and 5 . The ability of AGA to identify small deviations in healthy gait is investigated in chapter 4 using a simulation study of healthy subjects. The simulation allows identical deviations to be applied to healthy subjects in a standardised and controlled way, excluding the confounding effects of diseaserelated parameters that may influence gait (e.g. pain). Chapter $\mathbf{5}$ investigates the clinical validity of AGA on patients in orthopaedic practice. The gait pattern of patients indicated for total knee replacement is evaluated by AGA before and after surgery. In addition, an investigation is carried out to see whether AGA provides additional information about function beyond the standard clinical scales by comparing both functional assessment tools.

Chapters 6, 7 and 8 evaluate the third research question "Are gait parameters measured by acceleration-based gait analysis and the recovery responses to external perturbations in gait associated with fall risk?". Associations between the 
Tinetti scale, a commonly used subjective measure for fall risk, and objective gait characteristics as measured by AGA, are investigated in chapter 6. Gait of elderly subjects with different Tinetti scores is analysed and compared. The last two chapters focus on perturbations in gait pattern. An experimental trip set-up ("Timed Rapid impact Perturbation", TRiP) able to probe the human skeletal system with controlled perturbations of varying characteristics is developed and its design requirements are tested in chapter $\mathbf{7}$. Healthy young individuals are subjected to controlled perturbations in gait which vary in duration, force and obstruction time. A force sensor and 3D motion analysis are used to evaluate the design requirements of the TRiP (e.g. force, duration and obstruction time of the perturbation). Chapter 8 investigates how age, walking speed, muscle strengthand speed of information processing parameters are associated with the recovery response that individuals employ when they encounter a perturbation in gait. The gait pattern of healthy young and older subjects is therefore repeatedly perturbed using the TRiP set-up. Isometric knee extension and flexion strength are measured using a dynamometer and speed of information processing parameters are determined using a computer-based four-choice finger cuing reaction time task. The recovery response is evaluated (proportion perturbations recovered by ES) and associated with age, walking speed, muscle strength - and speed of information processing parameters.

Finally, the results of the above described studies and recommendations for future research are discussed in chapter 9. 


\section{RefERENCES}

1. Studenski S, Perera S, Patel K, Rosano C, Faulkner K, Inzitari M, et al. Gait Speed and Survival in Older Adults. JAMA 2011;305(1):50-8.

2. Dumurgier J, Elbaz A, Ducimetière $P$, Tavernier B, Alpérovitch A, Tzourio C. Slow walking speed and cardiovascular death in well functioning older adults: prospective cohort study. BMJ 2009;339:b4460-7.

3. Annegarn J, Meijer K, Lima Passos V, Stute K, Wiechert J, Savelberg HH, et al. Problematic activities of daily life are weakly associated with clinical characteristics in COPD. J Am Med Dir Assoc 2012;13(3):284-290.

4. Nigg BM, Fisher V, Ronsky J. Gait characteristics as a function of age and gender. Gait Posture $1994 ; 2(4): 213-20$.

5. Kerringan DC, Todd MK, Coroce UD. Gender differences in joint biomechanics during walking: normative study in young adults. Am J Phys Med Rehabil 1998;77:2-7.

6. Menz HB, Lord SR, Fitzpatrick RC. Acceleration patterns of the head and pelvis when walking are associated with risk of falling in community-dwelling older people. J Gerontol 2003;58a:446-52.

7. Trombetti A, Hars M, Hermann FR, Kressig RW, Ferrari S, Rizzoli R. Effect of music-based multitask training on gait, balance, and fall risk in elderly people: a randomized controlled trial. Arch Intern Med 2011;28;171(6):525-33.

8. Moe-Nilssen R, Helbostad JL. Estimation of gait cycle characteristics by trunk accelerometry. J Biomech 2004;37:121-6.

9. Menz HB, Lord SR, Fitzpatrick RC. Acceleration patterns of the head and pelvis when walking on level and irregular surfaces. Gait Posture 2003;18:35-46.

10. Wolf SI, Braatz F, Metaxiotis D, Armbrust P, Dreher T, Döderlein L, et al. Gait analysis may help to distinguish hereditary spastic paraplegia from cerebral palsy. Gait Posture 2011; 22(4):556;564.

11. Saleh M, Murdoch G. In defence of gait analysis. Observation and measurement in gait assessment. J Bone Joint Surg 1985;67B(2):237-40.

12. Moe-Nilssen R. Test-retest reliability of trunk accelerometry during standing and walking. Arch Phys Med Rehabil1998;79:1377-85.

13. Henriksen M, Lund H, Moe-Nilssen R, Bliddal H, B D-S. Test-retest reliability of trunk accelerometric gait analysis. Gait Posture 2004;19:288-97.

14. Auvinet B, Berrut G, Touzard C, Moutel L, Collet N, Chaleil D, et al. Reference data for normal subjects obtained with an accelerometric device. Gait Posture 2002;16:124-34.

15. Auvinet B, Chaleil D, Barrey E. Accelerometric gait analysis for use in hospital outpatients. Rev Rhum Engl Ed 1999;66(7):389-97.

16. Mansfield A, Lyons GM. The use of accelerometry to detect heel contacts events for use as a sensor in FES assisted walking. Medical Engineering \& Physics 2003;25:879-85.

17. Sabatini AM, Martelloni C, Scapellator S, Cavallo F. Assessment of walking features from foot inertial sensing. IEEE Trans Biomed Eng 2005;52(3):486-94.

18. Zijlstra W, Hof AL. Assessment of spatio-temporal gait parameters from trunk accelerations during human walking. Gait Posture 2003;18:1-10.

19. Zijlstra W. Assessment of spatio-temporal parameters during unconstrained walking. Eur J Appl Physiol 2004;92:39-44.

20. Moe-Nilssen R, Helbostad JL. Estimation of gait cycle characteristics by trunk accelerometry. J Biomech 2004;37:121-6.

21. Kavanagh JJ, Barrett RS, Morrison S. Upper body accelerations during walking in healthy young and elderly men. Gait Posture 2004;20:291-8.

22. Saremi K, Marehbian J, Yan X, Regnaux J, Elashoff R, Bussel B, et al. Reliability and validity of bilateral thigh and foot accelerometry measures of walking in healthy and hemiparetic subjects. Neurorehabilitation and Neural repair 2006;20(2):297-305.

23. Brandes M, Zijlstra W, Heikens S, van Lummel R, Rosenbaum D. Accelerometry based assessment of gait parameters in children. Gait Posture 2006;24:482-286.

24. Aminian K, Rezakhanlou K, De Andres E, Fritsch C, Leyvraz PF, Robert P. Temporal feature estimation during walking using miniature accelerometers: an analysis of gait improvement after hip arthroplasty. Med Biol Eng Comput 1999;37:686-91. 
25. Moe-Nilssen R, Helbostad JL, Talcott JB, Toennessen FE. Balance and gait in children with dyslexia. Exp Brain Res 2003;150:237-44.

26. Menz HB, Lord SR, Fitzpatrick RC. Acceleration patterns of the head and pelvis when walking are associated with risk of falling in community-dwelling older people. J Gerontology 2003;58A(5):44652.

27. Paquet JM AB, Chaleil D, Barrey E. Analysis of gait disorders in Parkinsons' disease assessed with an accelerometer. Rev Neurol 2003;159(8-9):786-9.

28. Auvinet B, Chaleil D, Barrey E. Accelerometric gait analysis for use in hospital outpatients. Rev Rhum Engl Ed 1999;66(7-9):389-97.

29. Sekine M, Akay M, Tamura T, Higashi Y, Fujimoto T. Fractal dynamics of body motion in patients with Parkinson's disease. J Neural Eng 2004;1(1):8-15.

30. Henriksen $M$, Lund $H$, Moe-Nilssen $R$, Bliddal $H$. Test-retest reliability of trunk accelerometric gait analysis. Gait Posture 2004;19:288-97.

31. Kavanagh JJ, Morrison S, James DA, Barrett R. Reliability of segmental accelerations measured using a new wireless gait analysis system. J Biomech 2006;39:2863-72.

32. Bautmans I, Jansen B, Keymolen van B, Mets T. Reliability and clinical correlates of 3Daccelerometry based gait analysis outcomes according to age and fall-risk. Gait Posture 2011; 33(3):366-372.

33. Hausdorff JM, Rios DA, Edelberg HK. Gait variability and fall risk in community-living older adults: a 1 year prospective study. Arch Phys Med Rehabil 2001;82:1050-6.

34. Berg WP, Alessio HM, Mills EM, Tong C. Circumstances and consequences of falls in independent community-dwelling older adults. Age Ageing 1997;26(4):261-8.

35. Srygley JM, Herman T, Giladi N, Hausdorff JM. Self-report of missteps in older adults: A valid proxy of fall risk? Arch Phys Med Rehabil 2009;90(5): 786-792.

36. Eng JJ, Winter DA, Patla AE. Strategies for recovery from a trip in early and late swing during human walking. Exp Brain Res 1994;102:339-49.

37. Pijnappels M. Push-off reactions in recovery after tripping discriminate young subjects, older nonfallers and older fallers. Gait Posture 2005;21(4):388-94.

38. Lamoureaux EL, Sparrow WA, Murphy A, Newton RU. The effects of improved strength on obstacle negotiation in community-living older adults. Gait Posture 2003;17:273-83.

39. Schillings AM, Mulder T, Duysens J. Stumbling over obstacles in older adults compared to young adults. J Neurophysiol. 2005;94:1158-68. 



\section{Chapter 2 \\ Acceleration-based gait test for healthy subjects: Reliability and reference data}

Senden R, Grimm B, Heyligers IC, Savelberg HHCM, Meijer K. Gait Posture 2009; 30 (2):192-196. 


\section{ABSTRACT}

Accelerometers enable us to analyse gait outside conventional gait laboratories. Before these devices can be used in large scale studies and in clinical settings a thorough evaluation of their performance in different populations is required. The aim of this study was to present an acceleration-based reference database for healthy gait. The repeatability and inter-observer reliability of acceleration-based gait analysis was investigated. The sensitivity was tested on different age groups and the effect of gender was studied. A comprehensive set of gait parameters (i.e. cadence, speed, asymmetry and irregularity) were studied in 60 women and 60 men. Basic gait parameters showed high repeatability (VC cadence $1.51 \%$, ICC cadence 0.996 ) and inter-observer reliability (ICC cadence 0.916 ), while asymmetry and irregularity showed lower repeatability (VC asymmetry $47.88 \%$, ICC asymmetry 0.787 ) and inter-observer reliability (ICC asymmetry 0.449 ). The effects of age and gender on gait parameters were found to be consistent with those reported in studies using other methodologies. These findings and the advantages of the device support the application of AGA for routine clinical use and in daily life. 


\section{INTRODUCTION}

As walking is a basic requirement for many daily activities, gait analysis provides important information on the functional capacity of subjects (1). In clinical practice, gait is analysed visually or using questionnaires, producing subjective and mainly pain-related functional results $(2,3)$. In research settings, advanced motion analysis systems are used to carry out quantitative gait analysis and evaluate the impact of age, gender and several pathologies on gait characteristics (4).

Several studies have investigated spatio-temporal, kinematic and kinetic data for substantial sample groups under standardised laboratory conditions using 3Dmotion analysis systems and/or force plates (5). Although these methods can provide objective evaluation of gait, they are impractical for routine clinical use. They require technically skilled personnel and the measurements are timeconsuming. Furthermore, most of these studies take place in laboratory settings, with smaller and shorter walkways than in daily life, creating unusual walking conditions (5-8). In addition, single stride analysis limits the determination of gait variability and symmetry which are considered important parameters for the evaluation of pathological gait.

Recently accelerometer-based gait analysis (AGA) has emerged as a potential alternative for conventional gait analysis because of its reliability in analysing gait parameters and lower limb motion $(9,10)$. Various clinically relevant gait parameters, such as cadence and walking variability, can simultaneously be derived from acceleration signals using autocorrelations or peak detection algorithms (1113). Studies comparing different attachment positions of the accelerometer on the body showed attachment at the sacrum to produce the most reliable accelerometer signals. It has been argued that this is because the sacrum is near the Centre of Mass (CoM) of the body and the trunk provides stable support for the head and neck during walking (14-16). Accelerometers are wireless, non-obtrusive and easy to use, making them suitable for use outside the laboratory, in clinical settings. A long hospital corridor with normal walkways, normal surroundings, without intimidating equipment etc. provides a more natural environment to measure gait than the usual laboratory setting. To conduct a single gait test, including setup, measurement and analysis, may take as little as $10 \mathrm{~min}$, permitting the examination of several subjects in a relatively short time period. In addition, AGA can be used for quick analysis of multiple steps, which allows us to test fluctuations in gait pattern resulting in the measurement of gait variables such as variability (17).

To apply AGA in clinical practice on patients with functional limitations, a large healthy sample group is needed for reference. Auvinet et al. used a tailor-made biaxial-accelerometer setup to collect reference data for common gait parameters like regularity and frequency in 282 healthy subjects (18). They showed that the 
portable system easily provides accurate evaluation of walking. Recently, commercial systems have become available, which incorporate sensors and acquisition hardware in small devices and use sampling rates of up to $100 \mathrm{~Hz}$ enhancing the capacity for evaluating clinically relevant parameters like asymmetry and irregularity (11). To make these systems applicable to clinical evaluation a reference database for healthy gait is needed based on these technologies.

The accuracy of AGA depends on several technical factors such as the attachment of the device, the exact measurement of the distance walked, instructions to the subject, the robustness of the algorithm, etc. Since gait is measured by several observers in clinical settings, the repeatability of AGA needs to be investigated.

This study aims to create a reference gait database for healthy subjects based on AGA including step length, cadence, speed, vertical displacement of the CoM, asymmetry (difference in successive step times) and irregularity (variability in subsequent steps of the same leg). The repeatability and inter-observer reliability of AGA was tested by repeating the measurement with the same subject, firstly on the same day with the same observer, and secondly on a second day with another observer. The resulting gait parameters were compared with previous published gait data.

\section{MethodS}

\section{Subjects}

Based on a self defined questionnaire assessing state of health, subjects were included who showed no neuromuscular, musculoskeletal or cardiovascular pathologies affecting their motion pattern. Only healthy subjects were chosen to ensure that the gait measured represented a healthy walking pattern. All subjects were able to walk without walking aids. Eight men and 16 women, ranging in age from 21 to 60 years, participated in the first study to establish the inter-observer reliability of AGA. For the main study, 120 volunteers (60 males, 60 females) ranging from 20 to 86 years of age were recruited for measurement. For each of the six age categories (20-29, 30-39, 40-49, 50-59, 60-69 and $\geq 70) 10$ female and 10 male participants were included. The study was approved by the local ethical committee; all participants gave their informed consent. Anthropometric measurements were collected (Table 1).

\section{Equipment}

Trunk accelerations were measured using a three-dimensional accelerometer (62 $\mathrm{mm} \pm 41 \mathrm{~mm} \pm 18 \mathrm{~mm}, 53 \mathrm{~g}, \mathrm{f}=100 \mathrm{~Hz}$, range: $\pm 2 \mathrm{~g}$, McRoberts $\mathrm{BV}$, The Hague, The Netherlands (11)). To avoid extraneous movement, this accelerometer was attached tightly to the skin at the level of the sacrum using adhesive tape. Data was 
Table 1: Demographics categorized by decade of age and sex.

\begin{tabular}{|c|c|c|c|c|c|c|c|c|c|}
\hline $\begin{array}{l}\text { Age } \\
\text { (yrs) }\end{array}$ & $M / F$ & $\begin{array}{l}\text { Weight } \\
\text { (kg) }\end{array}$ & $\begin{array}{c}\mathrm{p}- \\
\text { value }\end{array}$ & $\begin{array}{l}\text { Height } \\
\text { (m) }\end{array}$ & $\begin{array}{l}\mathrm{p}- \\
\text { value }\end{array}$ & Age (yrs) & $\begin{array}{l}\mathrm{p}- \\
\text { value }\end{array}$ & $\begin{array}{l}\text { BMI } \\
\left(\mathrm{kg} / \mathrm{m}^{2}\right)\end{array}$ & $\begin{array}{l}p- \\
\text { value }\end{array}$ \\
\hline \multirow[t]{2}{*}{$20 \geq 70$} & $\begin{array}{l}M \\
M=60\end{array}$ & $\begin{array}{l}81.25 \\
(12.08)\end{array}$ & $<0.01 *$ & $\begin{array}{l}1.80 \\
(0.07)\end{array}$ & $<0.01 *$ & $\begin{array}{l}49.55 \\
(17.67)\end{array}$ & 0.99 & $\begin{array}{l}25.05 \\
(3.85)\end{array}$ & 0.14 \\
\hline & $\begin{array}{l}w \\
n=60\end{array}$ & $\begin{array}{l}66.87 \\
(10.14)\end{array}$ & & $\begin{array}{l}1.67 \\
(0.06)\end{array}$ & & $\begin{array}{l}49.43 \\
(17.81)\end{array}$ & & $\begin{array}{l}24.10 \\
(3.38)\end{array}$ & \\
\hline \multirow[t]{2}{*}{$20-29$} & $\begin{array}{l}M \\
n=10\end{array}$ & $\begin{array}{l}72.50 \\
(8.89)\end{array}$ & $0.03^{*}$ & $\begin{array}{l}1.84 \\
(0.05)\end{array}$ & $<0.01 *$ & $\begin{array}{l}24.50 \\
(2.76)\end{array}$ & 1.00 & $\begin{array}{l}21.44 \\
(1.85)\end{array}$ & 0.42 \\
\hline & $\begin{array}{l}W \\
n=10\end{array}$ & $\begin{array}{l}63.70 \\
(7.30)\end{array}$ & & $\begin{array}{l}1.70 \\
(0.06)\end{array}$ & & $\begin{array}{l}24.50 \\
(3.03)\end{array}$ & & $\begin{array}{l}22.13 \\
(1.92)\end{array}$ & \\
\hline \multirow[t]{2}{*}{$30-39$} & $\begin{array}{l}M \\
n=10\end{array}$ & $\begin{array}{l}81.10 \\
(4.04)\end{array}$ & 0.09 & $\begin{array}{l}1.83 \\
(0.06)\end{array}$ & $<0.01 *$ & $\begin{array}{l}33.30 \\
(1.89)\end{array}$ & 0.19 & $\begin{array}{l}24.27 \\
(1.45)\end{array}$ & 0.40 \\
\hline & $\begin{array}{l}W \\
n=10\end{array}$ & $\begin{array}{l}71.80 \\
(14.70)\end{array}$ & & $\begin{array}{l}1.67 \\
(0.07)\end{array}$ & & $\begin{array}{l}35.00 \\
(3.50)\end{array}$ & & $\begin{array}{l}25.52 \\
(4.31)\end{array}$ & \\
\hline \multirow[t]{2}{*}{$40-49$} & $\begin{array}{l}M \\
n=10\end{array}$ & $\begin{array}{l}80.30 \\
(15.67)\end{array}$ & $0.01 *$ & $\begin{array}{l}1.79 \\
(0.06)\end{array}$ & $<0.01 *$ & $\begin{array}{l}45.70 \\
(3.23)\end{array}$ & 0.07 & $\begin{array}{l}25.34 \\
(6.61)\end{array}$ & 0.50 \\
\hline & $\begin{array}{l}W \\
n=10\end{array}$ & $\begin{array}{l}64.80 \\
(7.52)\end{array}$ & & $\begin{array}{l}1.68 \\
(0.06)\end{array}$ & & $\begin{array}{l}43.10 \\
(2.42)\end{array}$ & & $\begin{array}{l}22.87 \\
(1.97)\end{array}$ & \\
\hline \multirow[t]{2}{*}{$50-59$} & $\begin{array}{l}M \\
n=10\end{array}$ & $\begin{array}{l}89.50 \\
(13.44)\end{array}$ & $<0.01 *$ & $\begin{array}{l}1.82 \\
(0.07)\end{array}$ & $<0.01 *$ & $\begin{array}{l}54.60 \\
(3.10)\end{array}$ & 0.77 & $\begin{array}{l}27.04 \\
(3.22)\end{array}$ & $0.02 *$ \\
\hline & $\begin{array}{l}W \\
n=10\end{array}$ & $\begin{array}{l}63.40 \\
(9.49)\end{array}$ & & $\begin{array}{l}1.65 \\
(0.05)\end{array}$ & & $\begin{array}{l}54.20 \\
(2.86)\end{array}$ & & $\begin{array}{l}23.29 \\
(3.22)\end{array}$ & \\
\hline \multirow[t]{2}{*}{$60-69$} & $\begin{array}{l}M \\
n=10\end{array}$ & $\begin{array}{l}80.20 \\
(8.79)\end{array}$ & $0.03 *$ & $\begin{array}{l}1.77 \\
(0.04)\end{array}$ & $<0.01 *$ & $\begin{array}{l}64.60 \\
(2.59)\end{array}$ & 1.00 & $\begin{array}{l}25.50 \\
(2.51)\end{array}$ & 0.90 \\
\hline & $\begin{array}{l}W \\
n=10\end{array}$ & $\begin{array}{l}70.30 \\
(10.02)\end{array}$ & & $\begin{array}{l}1.66 \\
(0.06)\end{array}$ & & $\begin{array}{l}64.30 \\
(3.53)\end{array}$ & & $\begin{array}{l}25.67 \\
(3.59)\end{array}$ & \\
\hline \multirow[t]{2}{*}{$\geq 70$} & $\begin{array}{l}M \\
n=10\end{array}$ & $\begin{array}{l}83.90 \\
(13.68)\end{array}$ & $<0.01 *$ & $\begin{array}{l}1.77 \\
(0.08)\end{array}$ & $<0.01 *$ & $\begin{array}{l}74.50 \\
(5.52)\end{array}$ & 0.61 & $\begin{array}{l}26.69 \\
(2.88)\end{array}$ & 0.17 \\
\hline & $\begin{array}{l}w \\
n=10\end{array}$ & $\begin{array}{l}66.90 \\
(8.44)\end{array}$ & & $\begin{array}{l}1.64 \\
(0.05)\end{array}$ & & $\begin{array}{l}75.70 \\
(4.85)\end{array}$ & & $\begin{array}{l}24.83 \\
(2.89)\end{array}$ & \\
\hline
\end{tabular}

Averages (standard deviations) * sign difference between men (M) and women $(\mathrm{W}) ; \mathrm{p}<0.05$

stored on a local memory card (256 MB). The unit was powered by two AAA1.5 V batteries.

\section{Protocol}

Subjects walked a $20 \mathrm{~m}$ straight distance at preferred speed while wearing the accelerometer. Their last step had to take them beyond the $20 \mathrm{~m}$ mark. The additional distance was measured by a ruler to obtain the exact distance walked. After $20 \mathrm{~m}$ subjects turned around and walked back. This procedure was carried out three times. Repeatability of AGA was measured within these six walks ensuring that all factors such as attachment of the device and shoe wear were kept constant 
All subjects walked across linoleum flooring in the hospital and they wore their own, flat shoes without high heels.

For the first study, the complete exercise was repeated with the same individual on a second day with another observer to investigate the inter-observer reliability. For the main study, the gait test was carried out on one occasion, with the same observer.

\section{Data analysis}

Raw data was downloaded to a PC using specific software (Mira 1.9 Beta, McRoberts BV, The Hague, The Netherlands (11)) and uploaded to a web-based analysis application. Gait parameters were calculated by proprietary, non-disclosed algorithms of the manufacturer based on the algorithms by Brandes et al. (11). Only the most relevant gait parameters demonstrated in a previous study were used (19). The basic gait parameters considered were step length, cadence, speed, and step time. Other parameters were vertical displacement of CoM and left-right asymmetry, which was calculated as the difference between the left and right step time divided by the bilateral average. Gait irregularity was determined by the variability in successive steps of the same leg. There are different methods for calculating this variability $(20,21)$. In this study the standard deviations of the left and right step time were used. Averages of the parameters over the six walks were used for analysis. To minimise the inter-subject variation in gait data, the step length, cadence, speed and vertical displacement were scaled for leg length (estimated from body height) according to Hof (22). Five asymmetry values out of the 120 were removed as outliers due to miscalculations. The other values for these subjects were not outliers.

\section{Statistical analysis}

The repeatability of AGA, measured over the first and second walk of six successive walks, was tested using intra-class correlation coefficients (ICC). Variation coefficients (VC\%) were determined over the six successive trials to express the percentage variation in a subjects' gait between successive trials. The interobserver reliability was evaluated using ICCs to show the effect on gait output of taking measurements on a second day with another observer. ICCs $>0.75$, between $0.40-0.75$, and $<0.40$ were interpreted respectively as excellent, fair-to-good and poor inter-observer reliability (23). In additional, paired t-tests were performed to test for any systematic differences between the two measurement sessions. The Kolmogorov-Smirnov test was used to check the normality of the variables. Depending on the nature of the distribution, parametric and non-parametric tests were performed to test for differences between genders. All statistics was performed using SPSS version 15.0 and differences were considered significant if $p$ values were less than 0.05 . 


\section{RESULTS}

There was no difference between the overall average ages of men (49.6 \pm 17.7 years) and women ( $49.4 \pm 17.8$ years) or between the average ages of men and women in each group. In all age groups the men were significantly taller than the women (range: $1.77-1.84 \mathrm{~m}$ and $1.64-1.70 \mathrm{~m}$ respectively). Body weight was higher in men (range: 72.5 - $89.5 \mathrm{~kg}$ vs. $63.4-71.8 \mathrm{~kg}$ ), but this difference was not significant for subjects in the $30-40$ age group. BMI values were comparable for men and women (average of $25.1 \pm 3.9 \mathrm{~kg} / \mathrm{m}^{2}$ vs. $24.1 \pm 3.4 \mathrm{~kg} / \mathrm{m}^{2}$ ), with the exception of the 50-60 age group where men showed higher BMI values $(27.0 \pm 3.2$ $\mathrm{kg} / \mathrm{m}^{2}$ vs. $23.3 \pm 3.2 \mathrm{~kg} / \mathrm{m}^{2}$ ) (Table 1 ).

\section{Repeatability and inter-observer reliability}

The average values for most gait parameters were similar across both sessions. The paired t-test showed that there were no significant systematic differences ( $p$-value $<0.05$ ) in any of the gait parameters between both measurement days performed using different observers on the second day, indicating that gait was similar over time. The basic gait parameters (step length, cadence, speed, step time) showed high repeatability (VC 1.51 - 3.07\%; ICC 0.902 - 0.997) and excellent inter-observer reliability (ICC 0.774 - 0.916). Data showing high repeatability (VC 5.96\%; ICC 0.929) but fair-to-good inter-observer reliability (ICC 0.529) were obtained for the vertical displacement of the Centre of Mass. The irregularity and asymmetry showed lower repeatability (VC 29.25 - 47.88; ICC 0.509 - 0.787) and inter-observer reliability (ICC $0.010-0.351)$.

\section{Reference data}

In general, men took significantly larger steps $(0.80 \pm 0.08 \mathrm{~m}$ vs. $0.71 \pm 0.07 \mathrm{~m})$, showed significantly higher vertical displacement $(5.04 \pm 1.09 \mathrm{~cm}$ vs. $4.47 \pm 0.87$ $\mathrm{cm})$, significantly higher step time $(0.55 \pm 0.03 \mathrm{~s}$ vs. $0.51 \pm 0.03 \mathrm{~s})$, significantly faster speed $(1.49 \pm 0.20 \mathrm{~m} / \mathrm{s}$ vs. $1.40 \pm 0.17 \mathrm{~m} / \mathrm{s})$ and had significantly lower cadence ( 110.51 steps/min \pm 6.30 vs. $118.43 \pm 6.94$ steps $/ \mathrm{min}$ ) than women. Even when scaled for leg length, the differences between gender in step length and frequency remained significant, while the speed and vertical displacement corrected for leg length showed similar values in both gender (resp. $0.50 \pm 0.06$ vs. $0.49 \pm 0.06,0.06 \pm 0.01$ vs. $0.05 \pm 0.01$ ). The irregularities (resp. $0.017 \pm 0.008$, $0.016 \pm 0.008$ ) were comparable for men and women. These effects of gender on step length, step time, speed (except age $\geq 70$ years), cadence (except 20 - 29 age group), were observed in all age categories, although the differences were not always significant. A significantly reduced step length, cadence and speed were observed with increasing age (Table 2). These general age and gender effects are comparable with previous studies (Table 3 ). 


\section{Chapter 2}

Table 2: Gait parameters per age category for both genders.

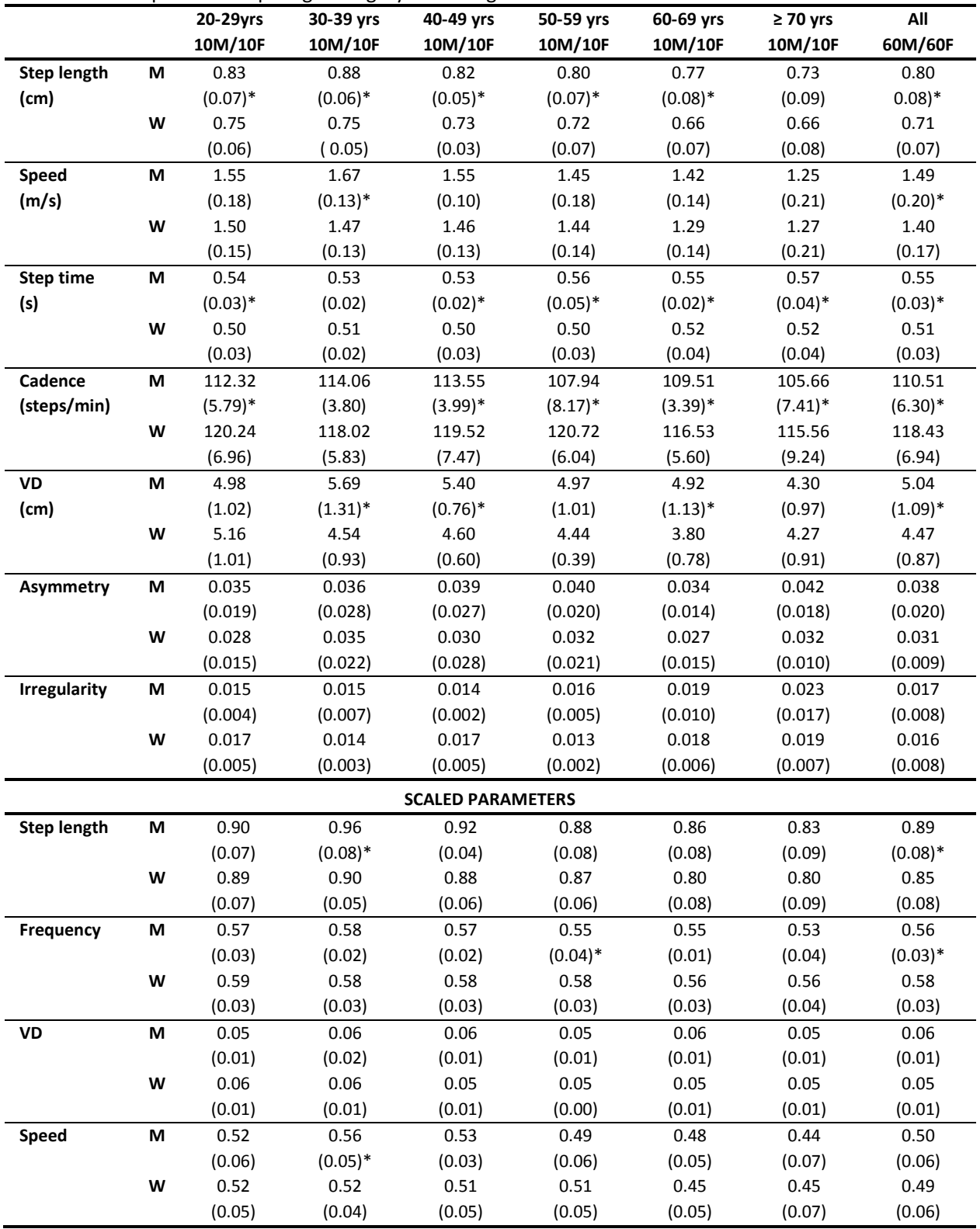

Averages (standard deviations) for the gait parameters. *sign difference between men (M) and women (W), $p<0.05$. VD; vertical displacement of Centre of Mass. 


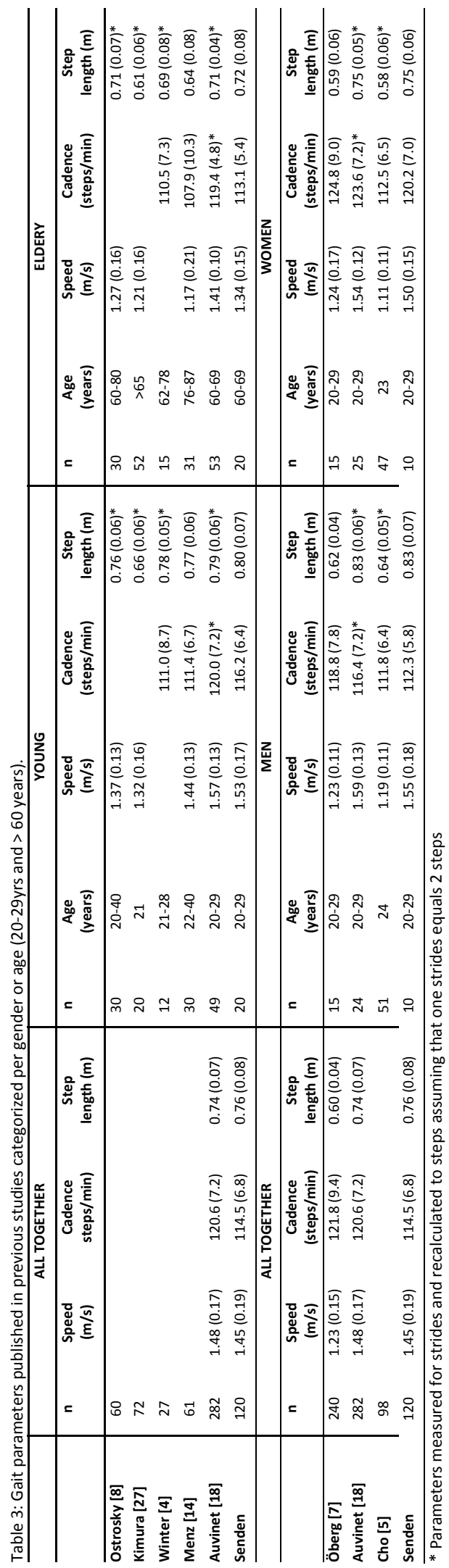




\section{Discussion}

The purpose of this study was to collect a reference database for healthy gait in men and women over different age groups using an accelerometer. First we investigated the repeatability and inter-observer reliability of AGA.

Results showed that AGA is repeatable for determining basic gait parameters such as speed and cadence in healthy subjects in non-laboratory situations such as hospital settings. The measurements obtained for vertical displacement, asymmetry and irregularity showed only fair-to-good repeatability and interobserver reliability. Basic gait parameters showed similar variations for age and gender to those reported in previous studies, making the accelerometer a suitable device for gait analysis in healthy subjects outside laboratory settings.

These results correspond to findings of other studies on AGA systems. Mackey et al. found that the IDEEA (system composed of five biaxial-accelerometers) showed high inter-session reliability (ICC 0.71 - 0.89). Lower intra-session reliability, measured using repeatability calculations, were shown for the IDEAA compared to three-dimensional gait analysis (10). In a test-retest setup, a triaxial AGA-test was shown to be reliable, showing ICC of 0.94 - 0.96 (15). Also, Maffiulett et al. found high intra-session reliability for an accelerometric gait analysis system (VC 2.73 $5.72 \%$; ICC 0.97 - 0.99) (9). Other motion analysis systems such as gyroscopes showed high intra- and inter-session reliability (ICC $0.94-0.98$ vs. ICC $0.91-0.95$ ) and low VCs (1.34 - 1.57\%) for cadence, speed, gait cycle time and stride length (24). Force plates showed VCs of $1.65-2.58 \%$ and ICCs of $0.99-0.99$ indicating high intra-session reliability (9). Our results were as good as those found in other studies and indicate that the production of basic gait parameters using AGA is robust and unaffected by differences in performance techniques used by different observers. This is an important requirement for clinical use where different observers have to conduct the gait test on several occasions.

Some parameters scored more poorly and are probably more sensitive to experimental conditions and device capability. The acquisition frequency of $100 \mathrm{~Hz}$ is a limiting factor in accurately determining gait parameters such as vertical displacement, asymmetry and irregularity. A low sampling rate increases the chance of producing temporal aliasing, especially in heel strike detection which needs to be precise, particularly for gait variability parameters like asymmetry and irregularity. Healthy gait is expected to be symmetric and regular, which is reflected in very low, near to zero, variability values. Because of the definition used to express the asymmetry and irregularity, a small change will result in a relatively big difference which may explain their low variance coefficients. Higher sampling frequencies are needed for these definitions of asymmetry and irregularity to resolve variability differences at 0.01 . Alternatively, other measures of variability could be used for more robust outcomes (25). For instance, Auvinet et al. used an 
accelerometer measuring with a sampling frequency of $50 \mathrm{~Hz}$ while calculating reliable asymmetry and irregular values by applying autocorrelations (18). One would expect gait asymmetry and irregularity to be more pronounced in pathological gait, assuming that these parameters can be used for group comparisons, for instance to identify pathological gait. However, more research is needed to investigate the use of these parameters for individual use and long term studies.

The basic gait data presented in the current study corresponded with previous published reports. The observation that men walk faster and take bigger steps, while having a lower cadence than women, is commonly reported in many studies using laboratory-based gait analysis systems (4, 5, 7, and 26). Moreover, the typical effects of age on basic gait parameters observed in previous studies, including slower speed, shorter step length and longer step times in the elderly, are also found in the current study $(4,8$, and 27$)$. Moreover, ageing is generally associated with decreased foot clearance which is adopted by the elderly to compensate for balance impairment (6). This characteristic corresponds to the small decrease in vertical displacement shown in the older population in our data. This parameter is relevant when analysing patients or the elderly because a higher vertical displacement while walking corresponds to higher energy expenditure, resulting in more effort to walk a certain distance. However, more research is needed on this topic because existing research about the relation between vertical displacement and walk efficiency is contradictory (29).

The collected reference data corresponded more closely with gait data produced using different acceleration-based techniques than with gait data produced by laboratory-based gait analysis systems. There was a high level of correspondence in basic gait parameters with the reference data for healthy subjects collected by Auvinet et al. who used two accelerometers (18). Only a slightly higher speed $(2.6 \%$ vs. $5.2 \%)$, cadence (3.3\% vs. $5.6 \%$ ) and a slightly shorter step length ( $1.3 \%$ vs. $1.4 \%)$ for young and older subjects was observed. Moreover, small differences ( 0 - 3.6\%) were observed when comparing the gait of men and women.

AGA-based studies analysing gait of younger (20 - 29 years) and older (60 - 69 years) subjects showed differences ranging from $1.4 \%-17.5 \%$ for step length, 5.8 $13.7 \%$ for speed and $2.3-4.6 \%$ for cadence compared to our reference data. Studies using laboratory-based method showed 17 - 26\% slower speed, 21 - 25\% shorter step length and $0.5-6.4 \%$ higher cadence compared with our data. These differences with our reference database can partly be attributed to the length of the walkway. A relatively long walkway $(20-28 \mathrm{~m})$, as is used in the current study, reflects normal conditions, while narrow and short walkways (e.g. $5.5 \mathrm{~m} \mathrm{(7)),} \mathrm{as}$ usually used in laboratory settings, requires the subject to start and brake frequently which has a relatively large effect on the walking pattern and its measurement. Moreover, several studies calculated gait parameters over a stride, 
which may explain the differences in step length between our database and lab based values $(5,18)$. Although some discrepancy was found between the collected reference database and data obtained with laboratory-based methods, the gait parameters of our database lie within the ranges that have been obtained for healthy individuals.

\section{Conclusion}

This study collected a reference database for healthy gait using an accelerationbased gait test. Repeatable basic gait output was obtained from the accelerometer which showed general comparability with the temporal-spatial gait parameters produced by validated advanced motion analysis systems. These promising results and the favourable characteristics of the accelerometer make the device suitable for use outside the laboratory, for instance in clinical settings. 


\section{REFERENCES}

1. Sadeghi $H$, Allard P, Prince F, Labelle H. Symmetry and limb dominance in able-bodied gait: a review. Gait Posture 2000;12:34-45.

2. Brunnekreef JJ, van Uden CJ, van Moorsel S, Kooloos JG. Reliability of video-taped observational gait analysis in patients with orthopaedic impairments. BMC-musculoskelet disord 2005;6:17-26.

3. Salch M, Murdoch $\mathrm{G}$. In defence of gait analysis. Observation and measurement in gait assessment. J Bone Joint Surg 1985;67B:237-41.

4. Winter DA, Patla AE, Frank JS, Walt SE. Biomechanical walking pattern changes in the fit and healthy elderly. Phys Ther 1990;70:340-7.

5. Cho SH, Park JM, Kwon OY. Gender differences in three dimensional gait analysis data from 98 healthy Korean adults. Clin Biomech 2004;19:145-52.

6. van Dieën J, Pijnappels M, Bobbert MF. Age related intrinsic limitations in preventing a trip and regaining balance after a trip. Saf Sci 2005;43:437-53.

7. Oberg T, Karsznia A, Oberg K. Basic gait parameters: reference data for normal subjects, 10-79 years of age. J Rehabil Res Dev 1993;30:210-23.

8. Ostrosky KM, Van Swearingen JM, Burdett ZG. A comparison of gait characteristics in younger and old subjects. Phys Ther 1994;74:637-46.

9. Maffiulett NA, Gorelick M, Kramers-de Quervain I, Bizzini M, Munzinger JP, Tomasetti S, et al. Concurrent validity and intrasession reliability of the IDEEA accelerometry system for the quantification of spatiotemporal gait parameters. Gait Posture 2008;27:160-3.

10. Mackey AH, Stott NS, Walt SE. Reliability and validity of an activity monitor (IDEEA) in the determination of temporal-spatial gait parameters in individuals with cerebral palsy. Gait Posture 2008;28:634-9.

11. Brandes M, Zijlstra W, Heikens S, van Lummel R, Rosenbaum D. Accelerometry based assessment of gait parameters in children. Gait Posture 2006;24:482-6.

12. Moe-Nilssen R, Helbostad JL. Estimation of gait cycle characteristics by trunk accelerometry. J Biomech 2004;37:121-6.

13. Zijlstra W, Hof AL. Assessment of spatio-temporal gait parameters from trunk accelerations during human walking. Gait Posture 2003;18:1-10.

14. Moe-Nilssen R. Test-retest reliability of trunk accelerometry during standing and walking. Arch Phys Med Rehabil 1998;79:1377-85.

15. Henriksen M, Lund H, Moe-Nilssen R, Bliddal H, Danneskiod-Samsoe B. Test-rest reliability of trunk accelerometric gait analysis. Gait Posture 2004;19:288-97.

16. Menz HB, Lord SR, Fritzpatrick RC. Acceleration patterns of the head and pelvis when walking on level and irregular surfaces. Gait Posture 2003;18:35-46.

17. Chau T, Young S, Redekop S. Managing variability in the summary and comparison of gait data. J Neuroeng Rehabil 2005;2:22-42.

18. Auvinet B, Berrut G, Touzard CML, Collet N, Chaleil D, Barrey E. Reference data for normal subjects obtained with an accelerometric device. Gait Posture 2002;16:124-34.

19. Grimm B, Senden R, Vanderhenst T. Application feasibility of accelerometer based gait analysis in clinical orthopaedics. J Biomech 2006;39:S110.

20. Owings TM, Grabiner MD. Step width variability, but not step length variability or step time variability discriminates gait of healthy young and older adults during treadmill locomotion. J Biomech 2004;37:935-8.

21. Hausdorff JM. Gait variability: Methods, modelling and meaning. J Neuroeng Rehabil 2005;2:19-28.

22. Hof AL. Scaling gait data to body size. Gait Posture 1996;4:222-3.

23. Shrout PE, Fleiss JL. Intraclass correlations: uses in assessing rater reliability. Psychol Bull 1979;86:420-8.

24. Allet L, Armand S, Bie de RA, Golay A, Monnin D, Aminian K, et al. Reliability of diabetic patients' gait parameters in a challenging environment. Gait Posture 2008;28:680-6.

25. Soudan K. Standardization of gait kinematic data using a gait symmetry index and Fourier analysis. In: Huiskes R, Van Camen DK, De Wijn JR, editors.

26. Biomechanics Principles and Applications Development in Biomechanics, vol.1. Martinus Nijhoff; 1982. p. 135-40. 


\section{Chapter 2}

27. Elble RJ, Sienko-Thomas S, Higgins C, Colliver J. Stride dependent changes in gait of older people. J Neurol 1991;238:1-5.

28. Kimura T, Kobayashi H, Nakayama E, Hanaoka M. Effects of aging on gait patterns in the healthy elderly. Anthropol Sci 2007;115:67-72.

29. Menz HB, Latt MD, Tiedemann A, Mun San Kwan M, Lord SR. Reliability of the Gaitrite walkway system for the quantification of temporal-spatial parameters of gait in young and older people. Gait Posture 2004;20:20-5.

30. Kuo AD. The six determinants of gait and the inverted pendulum analogy: Dynamic walking perspective. Hum Mov Sci 2007;26(4):617-56. 


\section{Chapter 3}

The importance of correcting for individual differences in the clinical diagnosis of gait disorders

Senden R, Meijer K, Heyligers IC, Savelberg HHCM, Grimm B Physiotherapy 2012;98:325-329 


\section{ABSTRACT}

This study aimed to quantify the effects of subject characteristics on gait parameters using acceleration-based gait analysis. 120 healthy subjects divided into six age groups of ten men and women per age decade, were asked to perform a $20 \mathrm{~m}$ walking test. Basic gait parameters (e.g. speed) and other clinically relevant parameters (e.g. step time asymmetry) were assessed during a $20 \mathrm{~m}$ walking test using a triaxial accelerometer, attached to the level of the sacrum. Subject characteristics (e.g. age, gender, height, body mass) were collected. $34 \%$ to $51 \%$ of the variability in basic gait parameters was explained by age, body height and gender. Subject characteristics contributed less to the variance in step time asymmetry $\left(R^{2} 0.02\right)$, gait irregularity $\left(R^{2} 0.07\right)$ and vertical displacement of Centre of Mass $\left(R^{2}\right.$ 0.17). The relations found were comparable to previous studies, e.g. showing faster walking speeds in men, younger and taller subjects. Age, body height and gender are determinants of basic gait parameters, while their influence on gait irregularity and step time asymmetry is minimal. This indicates that gait is variable between subjects showing the relevance to correct gait for subject characteristics. A start is made to build a database for healthy gait, correcting gait parameters simultaneously for age, gender and body height which will be relevant in clinical practice as it quickly put into perspective gait of patients. 


\section{INTRODUCTION}

Gait analysis is frequently used in clinical practice because it allows to investigate a subjects' walking capability, to assess the quality of gait, to detect deviations from normal walking, and thus to assist in therapeutic decision-making. Gait parameters (e.g. speed) are also useful clinical indicators of well being. Studies showed that walking speed is associated with survival (1) and cardiovascular mortality in elderly (2). In addition stride variability showed to be sensitive to identify elderly with an increased fall risk (3). In current clinical practice, gait is assessed by doctors' visual observation. This way of gait analysis lacks accuracy, is affected by observer bias and is incapable to identify small changes in gait. Acceleration-based gait analysis (AGA) is a relatively new objective gait analysis technique feasible for clinical use, which showed to be reliable and reproducible (4-6). In addition AGA is able to identify small changes in gait (4), showing its added value in clinical practice e.g. for diagnostics and evaluations.

For clinical purposes, a correct interpretation of the gait pattern is required. This needs insight in the determinants of gait and the dynamics of walking. Therefore many studies are done to investigate gait differences as a function of age, gender, body height or body weight. Most of these studies relied on sophisticated motion analysis systems (7-10), but no study used one single triaxial accelerometer to examine the effect of age, gender, body height and body mass on various gait parameters. However one recent study identified relationships between gait parameters and subject characteristics using an inertial sensor system (3D acceleroand gyrometer, attached at ankle) (11). Studies showed that gait changes with aging as was indicated by a slower walking speed, shorter step length, lower step frequency, lower foot clearance and wider step width in the elderly $(8,11)$. In addition typical gender differences in gait have been demonstrated. Men walk faster, taking longer steps and having a lower cadence compared to women (1112). These gender differences could partly be explained by differences in anatomy of the pelvis and hip and by differences in body height (men are generally bigger than women) (12-14). This indicates that gait is also influenced by body height. It is well known that small subjects take shorter steps and walk with higher step frequencies to keep up with taller subjects. Sutherland et al. even found that the ratio stride length to stature was similar for small and tall subjects, which indicated an effect of body height (15). Besides age, gender and body height, an effect of body mass has been shown. Studies even reported that the effect of variations in body mass was higher than the effect of variations in body height (16).

While the effects of subject characteristics on gait are generally known they have never been quantified. Only scaling methods have been introduced to eliminate the effect of body height or body mass on gait. For instance gait data in units of force, moment or power can be divided by body mass to correct for differences in body 
mass. Scaling methods for body height, which mainly rely on the biomechanical model representing gait as an inverted pendulum (16) compensate by dividing gait parameters such as step length and walking speed by body height or leg length (16). Hof et al. used leg length and gravity to correct for body height differences, obtaining a complete set of dimensionless parameters (17). In addition the Froude Number, a dimensionless measure of walking speed is commonly used to compare walking dynamics of subjects differing in stature (18). While these scaling methods are frequently applied in practice, they only cover the effect of body height either of body mass.

The current study aims to explore and quantify the effects of variations in age, gender, body height and body mass on various gait parameters, measured by the clinically feasible AGA system.

\section{Methods}

\section{Subjects}

One hundred and twenty healthy subjects, comprising sixty men and sixty women divided into six age groups of ten men and ten women per age decade, were included in the cross-sectional study with one single cohort group. Subjects had an average age of 49 years (20 -86 years), body height of $1.73 \pm 0.09 \mathrm{~m}$ and body mass of $74 \pm 13 \mathrm{~kg}$. The study was approved by the committee on research ethics. All participants were willing to participate and gave informed consent. The volunteers were considered healthy if they met the criteria summarized in the self-assessment checklist (Table 1).

\section{Procedure}

Each subject walked six times along a $20 \mathrm{~m}$ straight length of linoleum flooring in the hospital at self-selected speed. Trunk accelerations were measured in three orthogonal directions using a small, lightweight and portable triaxial accelerometer $(62 \times 41 \times 18 \mathrm{~mm}, 53 \mathrm{~g}, \mathrm{f}=100 \mathrm{~Hz}$, Dynaport Minimod, McRobertsBV, The Hague, The Netherlands, Fig. 1, (19)). The accelerometer was attached tightly to the skin at the level of the sacrum using adhesive tape while performing the gait test. After walking each $20 \mathrm{~m}$ length, the distance by which their last step extended beyond the $20 \mathrm{~m}$ marker was measured to obtain the exact distance walked. All subjects wore their regular shoes and normal clothing.

\section{Outcome measures}

Basic gait parameters such as step length, walking speed, cadence and other clinically relevant gait parameters such as the vertical displacement of the Centre of Mass (CoM), and irregularity [average (SD step time left; SD step time right)] 
Table 1: Self-assessment checklist used to include healthy subjects

\begin{tabular}{|c|c|c|}
\hline & FUNCTION BASED & Criteria \\
\hline 1. & Do you have problems with balancing? & no \\
\hline 2. & Do you have problems with walking? & no \\
\hline 3. & Do you have pain while walking & no \\
\hline \multirow[t]{2}{*}{4.} & Do you need walking aids while walking & no \\
\hline & HEALTH QUESTIONS & \\
\hline 5. & Do you suffer cardio-vascular problems? & no \\
\hline 6. & Do you suffer respiratory problems? & no \\
\hline 7. & Do you suffer musculoskeletal problems? (incl. osteoarthritis) & no \\
\hline 8. & Do you suffer neuromuscular or musculoskeletal pathologies? & no \\
\hline 9. & Are you a diabetic patient? & no \\
\hline 10. & Can you see clearly? & yes \\
\hline 11. & Are you frequently dizzy? & no \\
\hline \multirow[t]{2}{*}{12.} & Do you have lower back pain? & no \\
\hline & MEDICATION USE & \\
\hline 13. & Do you use more than 3 medications? & no \\
\hline 14. & $\begin{array}{l}\text { Do you use one of the following medications? Anti psychotics, psychoactive medication, } \\
\text { antidepressants, benzodiazepines, sedatives/hypnotics }\end{array}$ & no \\
\hline
\end{tabular}

were derived from the filtered anterior-posterior acceleration signal using integration processes and peak detection algorithms, analogous to those of Zijlstra and Brandes et al. (19). The left-right step time asymmetry [(mean step time left mean step time right)/ ((mean step time left + mean step time right)/2)*100] was calculated afterwards, using the derived step times. For each gait parameter, the average of the six walking trials was used for further statistical analysis.

\section{Statistical Analysis}

Multiple regression analysis, using the backward method, was done to explore the relations between age, gender, body mass, body height and gait parameters. Predictive models were build for walking speed, step length, cadence, vertical displacement of CoM, gait irregularity and step time asymmetry. All analyses were performed using SPSS 15.0. Differences were considered significant at $p<0.05$. 

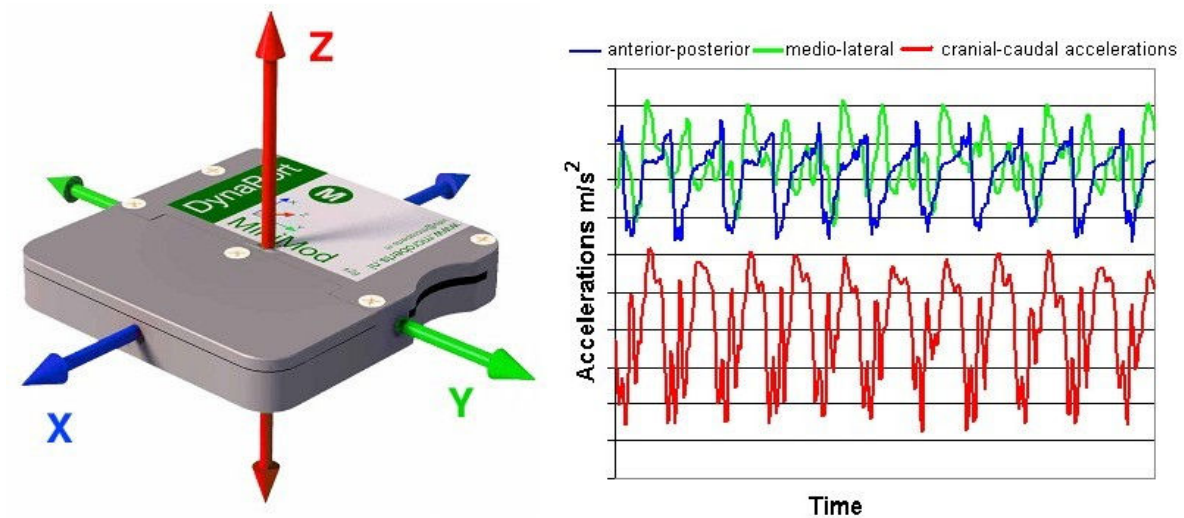

Fig. 1 Triaxial signal of walking, recognising individuals steps by peak detection.

\section{Results}

Significant predictive models were formulated for all gait parameters including age, gender and body height as determinants of gait. Body mass was not incorporated in any model (table 2). The step length was best predicted by subject characteristics $\left(R^{2}\right.$ of $\left.51 \%\right)$ including age $(B=-0.002)$, body height $(B=0.37)$ and gender $(B=-$ 0.043 ) as determinants. This indicates that for every unit increase in body height, a 0.37 unit increase in step length is predicted, holding all other variables constant. The explained variance for the cadence was $41 \%$, including age $(B=-0.175)$, body height $(B=-36.44)$ and gender $(B=2.97)$ as variables. Age $(B=-0.006)$ and gender $(B=-0.084)$ accounted for $34 \%$ of the variability in walking speed. The models for the vertical displacement of the CoM, gait irregularity and step time asymmetry were less related to subject characteristics $\left(R^{2} \leq 0.17\right)$. Age and body height were determinants of the vertical displacement and the gait irregularity. Step time asymmetry was only influenced by body height (Table 2 ).

Table 2: Multiple regression analyses with gait parameters as the dependent variables to determine the effect of subject characteristics. Vert. Displ.; Vertical displacement of center of mass.

\begin{tabular}{llcc}
\hline Parameter & Model & Adjusted $\mathbf{R}^{2}$ & $\mathbf{p}$-value \\
\hline Step length $(\mathrm{m})$ & $0.228-0.002 \times$ age $+0.370 \times$ body height $-0.043 \times$ gender & 0.51 & $<0.01$ \\
Cadence $($ steps $/ \mathrm{min})$ & $184.840-0.175 \times$ age $-36.437 \times$ body height $+2.970 \times$ gender & 0.41 & $<0.01$ \\
Speed $(\mathrm{m} / \mathrm{s})$ & $1.778-0.006 \times$ age $-0.084 \times$ gender & 0.34 & $<0.01$ \\
Vert. displ. $(\mathrm{m})$ & $-0.717-0.013 \times$ age $+3.532 \times$ body height & 0.17 & $<0.01$ \\
Gait irregularity (\%) & $-1.354+0.013 \times$ age $+1.378 \times$ body height & 0.07 & $<0.01$ \\
Gait Asymmetry (\%) & $15.585-6.900 \times$ body height & 0.02 & $<0.01$ \\
\hline
\end{tabular}




\section{Discussion}

The current study explored the relations between age, body height, body weight, gender and AGA-derived gait parameters. The magnitudes of the correlations appeared clinically important, even though they were modest in size. The fact that body height, age and gender together explained $34 \%$ to $51 \%$ of the variance in basic gait parameters, while gait is likely to depend on many more factors such as vision or environment conditions, indicates that these subject characteristics are clearly important. These percentages are comparable to studies which showed that age, gender, body height and body mass are independent predictors of the distance walked during a six-minute walk test in healthy subjects $\left(R^{2} 0.19-0.42\right)$ (20-21). In addition the subject characteristics explained the greatest portion of the variance for step length $\left(R^{2}\right.$ 0.51), which was also found in a recent study of Schwesig et. al. ( $R^{2} 0.49$ for stride length) (11). The fact that age and body height were incorporated in every model of the current study except one (resp. step time asymmetry and walking speed) shows that age and body height are main determinants of gait. In addition these models indicate that age (or body height) is predictive for gait parameters, even when the effect of the sex and body height (or age) is taken into account. Besides age and body height, a considerable effect of gender on basic gait parameters was shown.

The contribution of age was shown by a decreasing step length, walking speed, cadence and vertical displacement and an increasing gait irregularity with advancing age. These results are similar to previous studies which used other motion analysis techniques $(8,11)$. Body height was related to the step length and cadence and indicated that taller subjects walk with bigger steps and lower cadence which is also shown previously (12). In addition body height was positively related to the vertical displacements and gait irregularity and negatively to the step time asymmetry. Further analysis showed that age and body height are significantly correlated $(R=-0.24)$. However this relations shows that only $6 \%$ of body height can be explained by age indicating an independent effect of height. This emphasises the importance to correct gait for age and body height differences when comparing gait among subjects. Gender related to the basic parameters in a similar way as previously reported (11) and indicated that women walk with shorter steps, a higher cadence and a slower walking speed. The gender difference in walking speed can partly be explained by men's significantly taller height. This is also evident from further analysis showing that the average walking speed of men and women becomes similar ( 0.5 for men and 0.49 for women) after correcting for body height according to Hof (17). Similar findings were found by Sutherland et al (15). In addition gait parameters were not affected by body mass. This lack of relations may be due to the relatively narrow range of body mass of the included healthy population. Moreover Alexander et al. suggested that the effect of body 
weight is largely due to the effect of body height, since taller subjects are usual heavier (22). More research including lean and overweight subjects is needed to further investigate the effect of body mass on gait.

The subject characteristics accounted only for $2 \%$ and $7 \%$ of the variability in respectively the step time asymmetry and gait irregularity. This suggests that step time asymmetry and gait irregularity are less dependent of healthy subjects characteristics and will more likely be due to the influence of a pathology. Therefore when step time asymmetry and gait irregularity is noted, it may indicate functional abnormalities specific to the pathology (23).

At least $50 \%$ of the variation in gait parameters remained unexplained, suggesting that more factors determine gait. Studies demonstrated that factors like visual acuity, fear of falling, musculoskeletal capacity including muscular strength in performing everyday activities are involved (24-25). Integration of the effect of these factors could improve the prediction models. However this results in a database requiring many measurements making its application less practical for clinical use. Additionally, the fact that many factors are influencing gait indicates that gait is highly individual, which may show that a perfect match between subjects is difficult to achieve.

Based on the data collected for the current study, a start for a reference database was made correcting gait parameters simultaneously for the main determinants of gait: age, body height and gender. This database will give further insight in the dynamics of walking, clarifying the level and proportion in which gait is affected by subject characteristics. This insight will improve the interpretation of gait, and thus contribute to improved clinical diagnostics and follow up capabilities. In addition the database will be relevant in clinical practice as it quickly put into perspective gait of patients. However more subjects need to be included to get more insight in the distribution and preciseness of the averages and standard deviations of the gait parameters.

The current study had some limitations. Leg length was calculated based on body height, which may be imprecise. However this is a widely used and accepted method (14) which is easy and quick and in addition very practical for clinical use where body height is part of the standard clinical assessment. In addition, the variety in body mass was relatively small so that the full effect of body mass on gait may still be hidden. Finally, the inclusion criteria concerning health state may be too strong to obtain a representative group of elderly because they frequently break the strict health inclusion criteria without being considered pathologic due to common age-related issues that may be considered normal. However the fact that only healthy elderly following strict selection were included is also beneficial for the collected healthy database, which enables to track deviations from normal, also in the older population. 


\section{Conclusion}

Age, body height and gender are determinants of basic gait parameters, but their influence on gait irregularity and step time asymmetry is minimal. This indicates that gait is variable between subjects showing the importance to correct gait for subject characteristics. A start is made to build a database for healthy gait, correcting gait parameters simultaneously for age, gender and body height. A further extension of the database will be relevant in clinical practice as it quickly put into perspective gait of patients. 


\section{REfERENCES}

1. Studenski S, Perera S, Kushanger P, Rosano Caterina, Faulkner K, Inzitari M, et al. Gait speed and survival in older adults. JAMA 2011;305(1):50-8.

2. Dumurgier J, Elbaz A, Ducimetiere P, Tavernier B, Alperovitch A, C T. Slow walking speed and cardiovascular death in well functioning older adults: prospective cohort study. BMJ 2009;339.

3. Lamoth CJ, Deudekom van FJ, Campen van JP, Appels BA, Vris de OJ, Pijnappels M. Gait stability and variability measures show effects of impaired cognition and dual tasking in frail people. J NeuroEngineering and Rehabil 2011;8:2-10.

4. Senden R, Heyligers IC, Meijer K, Savelberg H, Grimm B. Acceleration-based motion analysis as a tool for rehabilitation: exploration in simulated functional knee limited walking conditions. Am J Phys Med Rehabil 2011;90(3):226-32.

5. Senden R, Grimm B, Heyligers IC, Savelberg HHCM, Meijer K. Acceleration-based gait test for healthy subjects: Reliability and reference data. Gait Posture 2009;30(2):192-6.

6. Senden R, Grimm B, Meijer K, Savelberg H, Heyligers IC. The importance to including objective functional outcomes in the clinical follow up of total knee arthroplasty patients. Knee 2010;18(5):306-311.

7. Kerringan DC, Todd MK, Croce UD. Gender differences in joint biomechanics during walking: normative study in young adults. Am J Phys Med Rehabil 1998;77:2-7.

8. Nigg BM, Fisher V, Ronsky JL. Gait characteristics as a function of age and gender. Gait Posture 1994;2(4):213-20.

9. Crenna P, Frigo C. Dynamics of the ankle joint analyzed through moment-angle loop during human walking: Gender and age effects. Hum Mov Sci 2011;30(6):1185-1198.

10. Chung MJ, Wang JM. the change of gait parameters during walking at different percentage of preferred walking speed for healthy adults aged 20-60 years. Gait Posture 2010;31(1):131-5.

11. Schwesig R, Leuchte S, Fischer D, Ullmann R, Kluttig A. Inertial sensor based reference gait data for healthy subjects. Gait Posture 2011;33:673-8.

12. Cho SH, Park JM, Kwon OY. Gender differences in three dimensional gait analysis data from 98 healthy Korean adults. Clin Biomech 2004;19:145-52.

13. Meent $\mathrm{H}$ van de, Jansen $\mathrm{H}$, Linde $\mathrm{H}$ van der. Study of the human pelvis using CAT scan: gender differences and anatomy of the ramus ossis ischii. Prosthet Orthot Int 2008;32(4):385-9.

14. Troosters T, Gosselink R, Decramer M. Six minute walking distance in healthy elderly subjects. Eur Respir J 1999;14:270-4.

15. Sutherland DH, Olshen RA, Cooper L, Woo SL. The development of mature walking. JBJS 1980;62(3):336-53.

16. Pierrynowski MR, Galea V. Enhancing the ability of gait analyses to differentiate between groups: scaling gait data to body size. Gait Posture 2001;13:193-201.

17. Hof AL. Scaling gait data to body size. Gait Posture 1996;4:222-3.

18. Zijlstra W, Prokop T, Berger W. Adaptability of leg movements during normal treadmill walking and split-belt walking in children. Gait Posture 1996;4:212-21.

19. Brandes M, Zijlstra W, Heikens S, van Lummel R, Rosenbaum D. Accelerometry based assessment of gait parameters in children. Gait Posture 2006;24:482-6.

20. Camarri B, Eastwood PR, Cecins NM, Thompson PJ, Jenkins S. Six minute walk distance in healthy subjects aged 55-75 years. Respiratory Medicine 2006;100:658-65.

21. Gibbons WJ, Fruchter N, Sloan S, Levy RD. Reference values for a multiple repetition 6-min walk test in healthy adults older than 20 years. J Cardiopulmonary Rehabil 1998;21:87-93

22. Alexander RM. Review: Models and the scaling of energy costs for locomotion. J Exp Biol 2004;208:1645-52.

23. Mizner RL, Snyder-Mackler L. Altered loading during walking and sit-to-stand is affected by quadriceps weakness after total knee arthroplasty. J Orthop Res 2005;23:1083-90.

24. Ruiz JR, Sui X, Lobelo F, Morrow JR, Jackson AW, Sjostrom M, et al. Association between muscular strength and mortality in men: prospective cohort study. BMJ 2008;337(12):a439.

25. Dieën van J, Pijnappels $M$, Bobbert MF. Age related intrinsic limitations in preventing a trip and regaining balance after a trip. Safety Science 2005;43:437-53. 


\section{Chapter 4}

\section{Acceleration-based motion analysis as a tool for rehabilitation: Exploration in simulated functional knee limited walking conditions}

Senden R, Heyligers IC, Meijer K, Savelberg HHCM, Grimm B Am J Phys Med Rehabil 2011; 90(3): 226-232. 


\section{ABstract}

The aim of this study was to investigate the ability of acceleration-based gait analysis to differentiate between normal gait and different simulated functional knee limitations of increasing severity. Gait of 48 healthy subjects was measured under four different walking conditions. Gait parameters (e.g. speed and asymmetry) were derived from trunk accelerations. Subjects walked a $20 \mathrm{~m}$ distance under four conditions: normal, simulated limited knee extension, simulated limited knee flexion, and simulated arthrodesis. The functional knee limitations were simulated using an adjustable knee brace on the right leg. Acceleration-based gait analysis detected acute gait changes (i.e. speed, step length, step duration, cadence, vertical displacement, asymmetry and irregularity) during the simulated functional knee limitations with high repeatability. The degree of change depended on the severity of the limitation, with the more severe limitations producing bigger changes in gait and the relative changes comparing well with literature values measured with laboratory-based motion analysis. Acceleration-based gait analysis is sensitive for different walking conditions. The easy and fast use, the production of objective gait characteristics and the ability to differentiate functional knee limitations suggest its suitability for clinical rehabilitation. 


\section{INTRODUCTION}

Patients with disorders at the lower limbs frequently have to cope with functional limitations such as a limited total range of knee motion during the swing phase and the loading phase of gait (1). To compensate for functional limitations and to regain pain-free locomotion, patients change their gait pattern. For instance, laboratorybased studies found a slower walking speed, shorter step length, higher cadence, and prolonged gait cycles in patients with lower limb problems such as total knee arthroplasty (2-4). To accurately diagnose and evaluate patients over time, it is important to objectively evaluate gait characteristics. Gait analysis has shown to be a reliable tool for rehabilitation (5). Because of the long setup times, the cost, the complexity, and the laboratory dependency of currently validated motion analysis systems, new functional and practical assessment tools are needed for routine clinical use.

Body fixed sensors such as accelerometers have shown to be useful for the quantification of gait (6-16). This ambulant technique is low cost, easy, and quick to use, allowing inexperienced people to use it in a usual clinical follow up situation (8). Acceleration-based gait analysis (AGA) uses peak detection algorithms, detecting heel strikes, to recognize steps $(7,9,10)$. Gait parameters, such as walking speed, cadence, step length, vertical displacement of centre of mass (CoM), asymmetry (difference in successive steps) and irregularity (variability in subsequent steps of the same leg) can subsequently be derived from trunk accelerations $(10,14,17)$. Mansfield and Lyons (18) showed the validity of accelerometer-based heel contact detection in nonpathologic and hemiplegic gait. In addition, the potential of accelerometers to evaluate gait has been demonstrated not only in healthy subjects $(8-10,16)$ but also in post-operative hip arthroplasty patients (6), subjects with diabetic peripheral neuropathy (13) and subjects having a prosthesis (12). However, in clinical populations, AGA is used mainly to study spatiotemporal gait parameters such as step time, walking speed, and step length $(6,12)$. Only a few studies included more complex gait parameters such as gait variability and asymmetry. However, such parameters could be of clinical relevance because they are highly associated with pathologies $(13,19)$.

The aim of the present study was to evaluate the ability of AGA to identify changes in gait parameters caused by pure functional knee limitations. A simulation study with healthy subjects was conducted to exclude the confounding effects of diseaserelated parameters that possibly influence gait, such as pain. This approach was previously performed by Mansfield and Lyons (18). Besides spatio-temporal parameters, we also considered the vertical displacement of CoM, step time asymmetry and gait variability. Additional to the aim, the repeatability of AGA to measure simulated gait deviations and the ability of AGA to differentiate between 
different simulated functional knee limitations was investigated. This study is an intermediate step toward clinical validation of AGA in knee patients.

\section{Methods}

\section{Subjects}

A total of 48 subjects, of which 22 were men (range age, 28 - 38 yrs; height, 1.70 $1.90 \mathrm{~m}$; body mass, 63 - $92 \mathrm{~kg}$ ) and 26 were women (range age, 19 - 60 yrs; height $1.58-1.77 \mathrm{~m}$; body mass, $50-94 \mathrm{~kg}$ ), participated in the study. All subjects were healthy without a history of musculoskeletal or neurologic gait disorder or any painful condition. Of the 48 subjects, 15 had a left dominant leg. The side of the dominant leg was identified by asking the subjects to write down with which leg they kicked the ball. Before participation in the study, all subjects gave informed consent approved by the Atrium MC Parkstad.

\section{Test Procedure}

In this study, different functional knee limitations were simulated subsequently in the same subject via inducing constraints in knee motion by adjustable braces (15). This approach of intra-subject repeated measurements was chosen to avoid confounding by inter-individual differences. Only healthy subjects were included to reduce the influence of medical factors to obtain well-defined types of gait deviations. An adjustable brace (Telescoping Cool TROM, Donjoy) was worn on the right leg to simulate three functional knee limitations. These limitations were presented in a fixed order, starting with a less severe limitation and finishing with more severe limitations: extension limitation (able to flex, limited to extend for $30^{\circ}$ ), flexion limitation (able to extend, but only $30^{\circ}$ of flexion allowed) and arthrodesis (leg is fixed in extension, only $5^{\circ}$ of flexion possible). The extension limitation is the less serious limitation because it affects only the stance phase of the affected leg. Flexion limitation is more serious because it not only disturbs the swing phase of the affected leg but also affects the stance phase of the contralateral leg, which has to compensate for the limited flexion. The arthrodesis condition is the most severe because it allows only five $5^{\circ}$ of knee motion. The simulated knee limitations intended to represent limitations in range of motion symptomatic with meniscal tears, distortion, corpora libera, or arthrosis (20-22). Gait was measured using a triaxial accelerometer $(5.6 \times 6.1 \times 1.5 \mathrm{~cm} ; 54 \mathrm{~g} ; \mathrm{f}=100$ $\mathrm{Hz}$; range, $\pm 2 \mathrm{~g}$; Dynaport Minimod, McRobertsBV, The Hague, the Netherlands (11)) attached to the skin near the sacrum using double-sided tape. In each condition, participants walked a $20 \mathrm{~m}$ straight distance six times at preferred speed. Before simulating the functional knee limitations, subjects walked with-out any intervention, which served as reference. Before measuring, subjects were given 
approximately $1 \mathrm{~min}$ to familiarize themselves with the experience of walking with a functional limitation imposed by the brace (15).

\section{Gait Parameters}

Nondisclosed peak detection algorithms of the accelerometer company, based on the inverted pendulum model of walking, were used to derive gait parameters from trunk accelerations measured during a $20 \mathrm{~m}$ gait test. This method was described by Zijlstra and Hof $(9,10)$ and has been proven to be accurate in assessing gait in nondisabled adults, children, and patients after total hip arthroplasty (6,9-11). Basic gait parameters were calculated, such as walking speed, cadence, step length, step time of the left leg, step time of the right leg, and the mean step time. The step time was measured from heel strike to heel strike. Additional relevant parameters with potential use in orthopaedic patients were calculated, such as vertical displacement of the CoM (defined as the mean difference between the maximum and minimum vertical displacement of the body) and irregularity(a measure to indicate gait variability, calculated as mean (standard deviation of step time left: standard deviation of the step time right)). The asymmetry was determined as the percentage difference in successive step times and was calculated using the step time of the right and left legs, produced by the algorithm of the company.

\section{Statistical AnAlysis}

The repeatability of AGA over the six walking trials was tested for each walking condition using intraclass correlation coefficients (ICCS). The reproducibility was classified according to the Landis and Koch (23) classification as excellent $(0.8<$ ICC $\leq 1)$, good $(0.6<\mathrm{ICC} \leq 0.8)$, fair $(0.4<\mathrm{ICC} \leq 0.6)$, poor $(0.2<\mathrm{ICC} \leq 0.4)$, and bad $(\leq$ 0.2 ). Changes in gait parameters caused by the functional limitations were statistically evaluated using repeated measures analysis of variance. Pairwise comparison was used to compare gait parameters between the simulated conditions. All analyses were performed using SPSS 15.0 and differences were considered significant at $\mathrm{p}<0.05$.

\section{Results}

For each walking condition, excellent ICCs (range, 0.85 - 0.94) were found for basic gait parameters (speed, step length, cadence, step time total, step time left, and step time right). Fair to excellent ICCs (range, 0.49 - 0.82) were found for the vertical displacement and the asymmetry. The irregularity showed ICCs ranging from 0.47 to 0.59 , showing fair repeatability (Table 1 ). 
Table 1: Intraclass correlation coefficients for gait parameters between the first two walks and the first and last walk.

\begin{tabular}{lcccccc}
\hline & \multicolumn{2}{c}{ EXTENSION } & \multicolumn{2}{c}{ FLEXION } & \multicolumn{3}{c}{ ARTHRODESIS } \\
& W1 vs. W2 & W1 vs. W6 & W1 vs. W2 & W1 vs. W6 & W1 vs. W2 & W1 vs. W6 \\
\hline Step length (m) & 0.95 & 0.87 & 0.95 & 0.91 & 0.93 \\
Cadence (Hz) & 0.97 & 0.90 & 0.97 & 0.85 & 0.91 \\
Vert. displ. (cm) & 0.88 & 0.77 & 0.80 & 0.78 & 0.91 \\
Walk speed (m/s) & 0.95 & 0.84 & 0.95 & 0.86 & 0.93 \\
Step time left (s) & 0.94 & 0.77 & 0.93 & 0.77 & 0.91 \\
Step time right (s) & 0.94 & 0.88 & 0.94 & 0.82 & 0.92 \\
Step time total (s) & 0.96 & 0.88 & 0.97 & 0.83 & 0.91 \\
Irregularity & 0.59 & 0.41 & 0.47 & 0.37 & 0.80 \\
Asymmetry (\%) & 0.72 & 0.12 & 0.85 & 0.61 & 0.38 \\
\hline
\end{tabular}

Vert. displ.: vertical displacement

Table 2: Gait parameters measured during normal and three simulated walking conditions.

\begin{tabular}{lccccccccccc}
\hline $\mathbf{n}=\mathbf{4 8}$ & \multicolumn{2}{c}{ Normal } & \multicolumn{2}{c}{ Extension limitation } & \multicolumn{3}{c}{ Flexion limitation } & \multicolumn{2}{c}{ Arthrodesis } \\
\hline & mean & SD & mean & SD & & mean & SD & & mean & SD \\
\hline Step length (m) & 0.80 & 0.07 & 0.74 & 0.09 & $*$ & 0.73 & 0.10 & $*$ & 0.73 & 0.09 & $*$ \\
Cadence (steps/min) & 116.06 & 7.11 & 112.66 & 7.00 & $*$ & 107.78 & 7.32 & $*$ & 109.08 & 7.42 & $*$ \\
Vert. displ. (cm) & 4.99 & 1.03 & 5.06 & 1.12 & & 5.88 & 1.18 & $*$ & 6.12 & 1.07 & $*$ \\
Speed (m/s) & 1.54 & 0.16 & 1.37 & 0.19 & $*$ & 1.31 & 0.20 & $*$ & 1.33 & 0.19 & $*$ \\
Step number & 21.81 & 2.23 & 24.20 & 3.36 & $*$ & 24.28 & 3.48 & $*$ & 24.27 & 3.34 & $*$ \\
Step time (s) & 0.52 & 0.03 & 0.54 & 0.03 & $*$ & 0.56 & 0.04 & $*$ & 0.55 & 0.04 & $*$ \\
Step time L (s) & 0.53 & 0.04 & 0.54 & 0.04 & $*$ & 0.59 & 0.05 & $*$ & 0.59 & 0.05 & $*$ \\
Step time R (s) & 0.51 & 0.03 & 0.53 & 0.04 & $*$ & 0.53 & 0.04 & $*$ & 0.52 & 0.04 & $*$ \\
Asymmetry (\%) & 3.58 & 2.90 & 4.99 & 3.37 & $*$ & 12.18 & 7.07 & $*$ & 13.05 & 7.30 & $*$ \\
Irregularity & 1.31 & 0.35 & 1.65 & 0.38 & $*$ & 1.97 & 0.58 & $*$ & 1.94 & 0.50 & $*$ \\
\hline
\end{tabular}

* significant $(p<0.05)$ change with regards to normal gait.

The analysis of variance showed a significant effect of the simulated conditions on all gait parameters. With reference to the normal unlimited walking condition, every simulated functional knee limitation caused an increase in step time, asymmetry, irregularity, and vertical displacement of the CoM and a decrease in step length, cadence and walking velocity (Table 2). Almost all of these adaptations with respect to the normal walking condition were significant, except the increase in vertical displacement as a result of extension limitation (relative change of 3\%) and the increase in step time of the affected leg (right) as a result of arthrodesis (relative change of $2 \%$; Table 2 ).

Investigating the degree to which gait changed showed that the more severe the limitation, the bigger the change in gait (Table 2). For instance, during the extension limitation, the cadence was 3\% lower than during normal walking condition and further reduced until $9 \%$ during the most severe limitations. In addition, the asymmetry increased from $4 \%$ to $5 \%$ during the extension limitation condition, tripled to $12 \%$ during flexion limitation, and increased still more to $13 \%$ during the arthrodesis condition. The cadence, total step time, and vertical displacement differed significantly among all simulated conditions (extension 

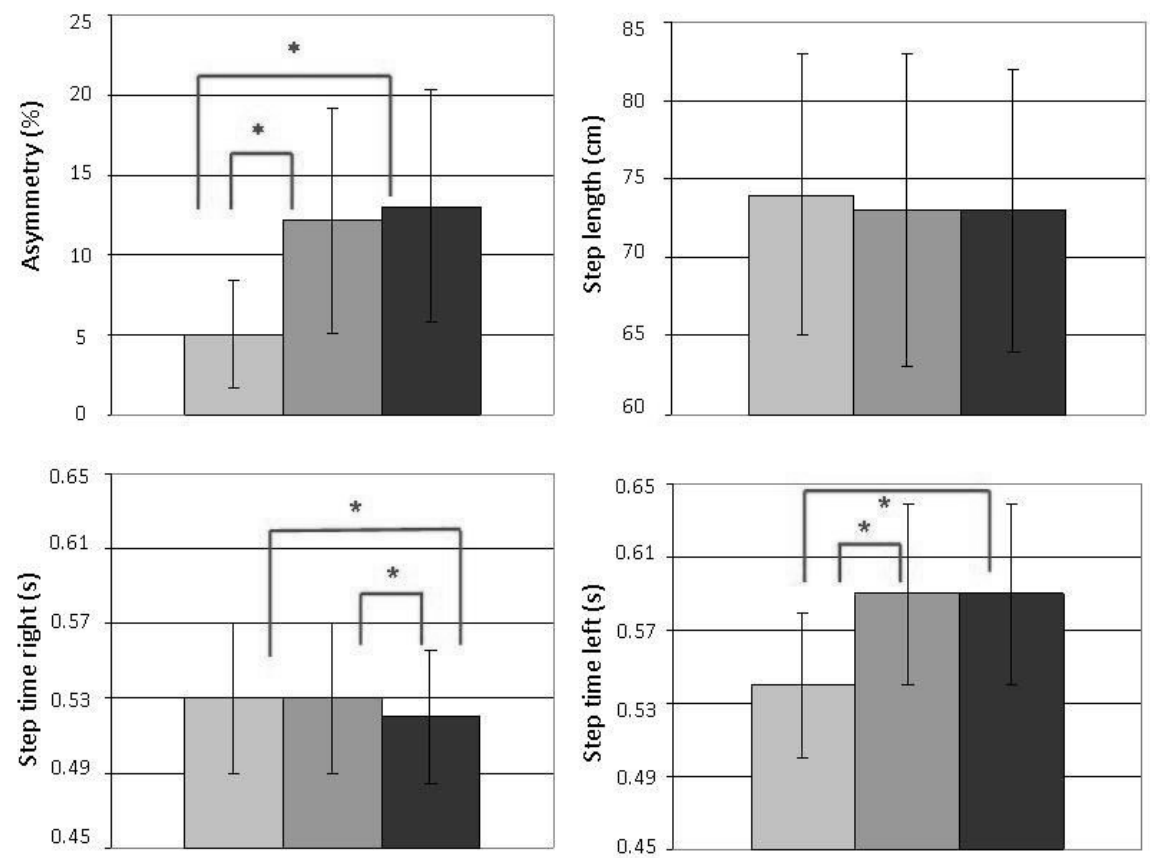

Extension limitation

Flexion limitation

Arthrodesis

Fig. 1: Comparison of gait parameters between the simulated knee limitations. ${ }^{*}$ significant difference $(p<0.05)$ between two simulated conditions. $L$ indicates left; $R$, right.

limitation vs. flexion limitation vs. arthrodesis). Step length was quite similar among the three simulated conditions. The flexion limitation and arthrodesis condition showed similar walking speed, asymmetry, step time left, and irregularity, whereas significant differences were found in comparison with the extension limitation condition. In contrast, the step time of the right leg was similar during extension and flexion limitation but significantly different from the arthrodesis limitation (Fig. 1).

\section{Discussion}

The current study evaluated the sensitivity of AGA to measure changes in gait in different gait conditions. In addition, the repeatability of AGA to measure simulated gait deviations and the ability of AGA to differentiate between simulated functional knee limitations were investigated.

AGA was able to differentiate the simulated functional knee limited walking conditions from normal gait. Acute gait changes were detected, which were 
comparable with previous studies analyzing the gait of knee patients using laboratory-based motion analysis systems. It has been shown that knee patients adapt their gait pattern to regain stability $(2,20,24)$. Studies reported a $13 \%$ reduced speed in patients with moderate osteoarthritis (25), a 9\% decreased cadence in patients with rheumatoid arthritis (26), and a 9\% decrease in stride length in patients operated for severe primary degenerative arthritis of a single knee joint (3). Quite the same changes were measured with AGA during the simulated functional knee limitations, which measured a $10 \%-14 \%$ decreased walking speed, a 3\% - 7\% reduced cadence, and a $7 \%-9 \%$ reduced step length. The ability of AGA to identify gait changes caused by different functional knee limitations, together with the fact that similar changes were found as reported in other studies, suggests that AGA is suitable in clinical practice with subjects with knee limitations. However further research is needed with knee patients to demonstrate this. In addition, AGA can detect small changes, such as a $1 \mathrm{~cm}$ decrease in step length, as is found between the simulated extension and flexion limitation condition. AGA is also able to identify changes that are not detectable by means of visual observation, such as the movements of the CoM, which increases by $1 \mathrm{~cm}$ during the simulated flexion limitation compared with the normal walking condition. The fact that AGA measures small and unobservable changes in gait shows the added value of an objective method to assess function.

AGA produced gait parameters with high repeatability during every walking condition, indicating that the gait performance was similar over successive trials. In addition, it shows that AGA is reliable at measuring disturbed gait. However, the gait parameter irregularity was produced with a relatively low repeatability. This either points to variations in step irregularity over several successive trials or indicates that AGA is less able to give reliable results for this measure. A larger variation in performance with respect to regularity could be attributed to the unusual movement behaviour evoked in healthy subjects, which causes an unstable and variable gait pattern. In addition, systematic errors, such as a learning effect emanating from an improved performance with repeated trials, might be causing inter-trial variations. The low ability of AGA to produce irregularity values can be caused by device limitations (e.g. sample frequency) or the sensitivity of the parameters to errors (e.g. peak detection algorithm) as previously reported (16). Further research involving real patients is needed to investigate the clinical value of the irregularity, which seems important because the irregularity is expected to be affected in pathologic gait.

The AGA output also provided insight into the compensatory strategy used to cope with the acute functional limitations. Both the affected and the unaffected leg needed more time to take a step during the simulated functional knee limitations, which is related to the slower walking speed. However, the increase in step time of the unaffected leg (left, $2 \%$ - 13\%) was higher than the increase in step time of the 
affected leg (right, 1\% - 4\%). This caused the affected leg to be longer in the swing phase and the unaffected leg to be longer in the stand phase during knee limitations, as was shown by the higher average step times of the unaffected leg during the flexion limitation and arthrodesis conditions (mean \pm SD, $0.59 \pm 0.05 \mathrm{~s}$ ) compared with the affected leg $(0.53 \pm 0.04 \mathrm{~s})$. This suggests that the unaffected leg has to compensate for the functional limitation applied on the contra-lateral leg by unloading the affected leg as much as possible. This difference in movement time between both legs resulted in an asymmetrical gait pattern, which was also identified by AGA during the simulated conditions. This indicates the ability of AGA to measure differences in leg behaviour, which is typical for unilateral pathologies and therefore important in clinical rehabilitation (19).

The degree to which gait changed depended on the severity of the limitation. The biggest gait adaptations were observed while simulating the most severe knee limitations (flexion limitation and knee arthrodesis), whereas gait was less affected during the simulated extension limitation, which is a less serious knee restriction. Comparative studies using laboratory-based gait analysis systems showed a $3 \%$ reduced cadence in patients planned for partial medial mensicectomy (27), a $9 \%$ lower cadence in patients receiving bilateral total knee arthroplasty (28) and a $21 \%$ decreased cadence in above-knee amputees (29), all respectively indicating an increased gait adaptation with increasing pathologic severity. This was also shown by the pairwise comparisons, where walking speed, left step time, asymmetry, and irregularity significantly differed between the simulated extension limitation and the two more severe simulated functional limited conditions, whereas these parameters were similar in the two more severe simulated limitations. The significant differences in the cadence and vertical displacement between all simulated conditions indicate that these parameters are most sensitive toward differentiation between simulated functional limitations. The fact that no significant differences were found in step length between the different simulated conditions indicates that these parameters are less sensitive to differentiation between acute functional limitations. This is also shown by the comparable reductions in stride length in patients with meniscal tear (11\%) (27), preoperative total knee arthroplasty patients (9\%) (30), and above-knee amputees (7\%) (29). It seems that there are gait parameters that are affected by knee pathologies in similar ways independent of the problem (e.g. step length) and others that can differentiate the problem (e.g. vertical displacement and cadence).

In general, every simulated functional knee limitation caused gait to change in the same direction. However, there were some inter-individual differences, hinting at individual gait patterns, adaptations, and compensation mechanisms, showing variability between subjects. The $1 \mathrm{~min}$ adaptation time used in this study may have been too short to allow subjects to become accustomed to the knee 
limitation, causing variations and both overestimation and underestimation in gait adaptations.

Real patients choose an alternative gait pattern over a longer period of time that best fits their requirements, which results in more efficient and pain-free locomotion. In addition, patients with knee problems usually have to deal with pain and other medical factors, which can lead to less or more limited movement and even higher changes in gait, indicating that the simulated conditions were not fully representative for patients with knee limitations. The current study showed the ability of AGA to detect differences in gait with an obvious deviation (e.g. limitation of $30^{\circ}$ ). For clinical purposes, it is interesting to investigate the limits of detection of AGA. Therefore, future studies should include a systematic variation of functional knee limitations in small steps.

\section{CONCLUSION}

The current study showed that AGA is sensitive to detect changes from healthy gait with high repeatability. The easy and fast production of objective and clinically important gait parameters, the detection of small gait changes, and the ability to differentiate different simulated functional knee limitations show its added value in clinical practice, for instance, to evaluate and assess individual rehabilitation programs. This study was an intermediate step toward clinical validation showing that AGA is able to measure disturbed gait of subjects with knee limitations. Further research is needed to investigate the suitability of AGA in clinical practice with knee patients. 


\section{REFERENCES}

1. McClelland JA, Webster KE, Feller JA: Gait analysis of patients following total knee replacement: a systematic review. The Knee 2007;14:253-63.

2. Bolanos AA, Colizza WA, McCann PD, et al: A comparison of isokinetic strength testing and gait analysis in patients with posterior cruciate-retaining and substituting knee arthroplasties. J Arthroplasty 1998; 13:906-15.

3. Fuchs S, Floren M, Skwara A, et al: Quantitative gait analysis in unconstrained total knee arthroplasty patients. Int J Rehabil Res 2002;25:65-70.

4. Fuchs $\mathrm{S}$, Tibesku $\mathrm{CO}$, Genkinger $\mathrm{M}$, et al: Clinical and functional comparison of bicondylar sledge prostheses retaining all ligaments and constrained total knee replacement. Clin Biomech 2004;19:263-9.

5. Tenore N, Fortugno F, Viola F, et al: Gait analysis as a reliable tool for rehabilitation of chronic hemiplegic patients. Clin Exp Hypertens 2006;28:349-55.

6. Akker-Scheek van den I, Stevens M, Bulstra SK, et al: Recovery of gait after short-stay total hip arthroplasty. J Arch Phys Med Rehabil 2007;88:361-7.

7. Auvinet B, Berrut G, Touzard CML, et al: Reference data for normal subjects obtained with an accelerometric device. Gait Posture 2002;16:124-34.

8. Kavanagh JJ, Menz HB: Accelerometry: a technique for quantifying movement patterns during walking. Gait Posture 2008;28:1-15.

9. Zijlstra W: Assessment of spatio-temporal parameters during unconstrained walking. Eur J Appl Physiol 2004;92:39-44.

10. Zijlstra W, Hof AL: Assessment of spatio-temporal gait parameters from trunk accelerations during human walking. Gait Posture 2003;18:1-10.

11. Brandes M, Zijlstra W, Heikens S, et al: Accelerometry based assessment of gait parameters in children. Gait Posture 2006;24:482-6.

12. Houdijk HAF, Velzen van JM, Woude van der LHV, et al: Validity of DynaPort GaitMonitor for assessment of spatiotemporal parameters in amputee gait. J Rehabil Res Dev 2008;45:1335-42.

13. Menz HB, Lord SR, St George R, et al: Walking stability and sensorimotor function in older people with diabetic peripheral neuropathy. Arch Phys Med Rehabil 2004;85:245-52.

14. Moe-Nillsen R: Test-retest reliability of trunk accelerometry during standing and walking. Arch Phys Med Rehabil 1998;79:1377-85.

15. Grimm B, Vanderherst T, Munch C, et al: Application feasibility of accelerometer based gait analysis in clinical orthopaedics. J Biomech 2006;39:S110.

16. Senden R, Grimm B, Heyligers ICC, et al: Acceleration-based gait test for healthy subjects: reliability and reference data. Gait Posture 2009;30:192-6.

17. Menz HB, Lord SR, Fitzpatrick RC: Acceleration patterns of the head and pelvis when walking are associated with risk of falling in community-dwelling older people. J Gerontol A Biol Sci Med Sci 2003; 58:446-52.

18. Mansfield A, Lyons GM: The use of accelerometry to detect heel contact events for use as a sensor in RES assisted walking. Med Eng Phys 2003;25:879-85.

19. Mizner RL, Snyder-Mackler L: Altered loading during walking and sit-to-stand is affected by quadriceps weakness after total knee arthroplasty. J Orthop Res 2005;23:1083-90.

20. Argenson AN, Vinel H, Aubaniac JM: Total knee arthroplasty for stiff knee. Interact Surg 2008;3:348.

21. Harato K, Otani T, Nakayama N, et al: When does postoperative standing function after total knee arthroplasty improve beyond preoperative level of function. The Knee 2009;16:112-25.

22. Myles CM, Rowe PJ, Walker CRC, et al: Knee joint functional range of movement prior to and following total knee arthroplasty measured using flexible electrogoniomtery. Gait Posture 2002;16:46-54.

23. Landis JR, Koch GG: The measurement of observer agreement for categorical data. Biometrics 1977; 33:159-74.

24. Liebensteiner MC, Herten A, Gstoettner M, et al: Correlation between objective gait parameters and subjective score measurements before and after total knee arthroplasty. The Knee 2008;15:461-6. 


\section{Chapter 4}

25. Mc Kean KA, Landry SC, Hubley-Kozey CL, et al: Gender differences exist in osteoarthritic gait. Clin Biomech 2007;22:400-9.

26. Weiss RJ, Wretenberg P, Stark A, et al: Gait pattern in rheumatoid arthritis. Gait Posture 2008;28:229-34.

27. Durand A, Richards CL, Malouin F, et al: Motor recovery after arthroscopic partial meniscectomy. Analyses of gait and the ascent and descent of stairs. J Bone Joint Surg Am 1993;75:202-14.

28. Solak AS, Kentel B, Ates Y: Does bilateral total knee arthroplasty affect gait in women? J Arthroplasty 2005;20:745-50.

29. Bae TS, Choi K, Mun M: Level walking and stair climbing gait in above-knee amputees. J Med Eng Technol 2009;33:130-5.

30. Smith AJ, Lloyd DG, Wood DJ: Pre-surgery knee joint loading patterns during walking predict the presence and severity of anterior knee pain after total knee arthroplasty. J Orthop Res 2004;22:2606 


\section{Chapter 5 \\ The importance to include objective functional outcomes in the clinical follow up of total knee arthroplasty patients}

Senden R, Grimm B, Meijer K, Savelberg HHCM, Heyligers IC. The Knee 2011; 18(5): 306-311 


\section{ABSTRACT}

In clinical practice, it is increasingly important to assess patients' daily functionality routinely and objectively. Acceleration-based gait analysis (AGA) has shown to be reliable and technically suitable for routine clinical use outside the laboratory. This study investigated the suitability of AGA for measuring function in orthopaedic patients with symptomatic gonarthrosis listed for total knee arthroplasty (TKA) by investigating (a) the ability of AGA to distinguish patients from healthy subjects, (b) the sensitivity to gait changes of AGA in assessing recovery following total knee arthroplasty in a subpopulation, and (c) correlations between AGA parameters and clinical scales. Gait was assessed using AGA in 24 patients with symptomatic gonarthrosis listed for TKA, and in 24 healthy subjects. AGA parameters (e.g. speed, asymmetry) and clinical scales (e.g. KSS) were used to monitor progress in 12 patients 3 months after TKA. The Mann-Whitney-U test, Receiver Operating Characteristic (ROC) curves, repeated measurement ANOVA and Pearson correlations were performed. AGA differentiated pathological from healthy gait. The area under the ROC curve, sensitivity and specificity values were high for speed, step frequency and step length. Different recovery profiles were found, with clinical scales showing faster recovery rates. None or only weak correlations were found between AGA and clinical scores. AGA was found to be of clinical relevance in identifying and monitoring patients with symptomatic gonarthrosis in orthopaedic practice, providing objective and additional information about function beyond clinical scales. This, together with the fact that AGA can be applied routinely, suggests the suitability of AGA for use in rehabilitation programs. 


\section{INTRODUCTION}

Pain relief and the improvement of knee function are the two main reasons for total knee arthroplasty (TKA) $(1,2)$. It has recently been shown that for the new generation of TKA patients, who are younger and much more demanding, function becomes increasingly important (3). Patients expect optimal functional levels after surgery. This makes it important to supervise the recovery process carefully and monitor the outcome of surgery, and to assess functionality in daily life frequently. Clinical outcome scales, such as, for example, the Western Ontario and McMaster University Osteoarthritis Index (Womac) and the Knee Society Score (KSS), are popular in clinical practice for function assessment because they are non-invasive and easy to use (4). The perceived mobility status during daily activities is assessed by questionnaire, resulting in clinical outcome scales that are pain- dominated and subjective. This may mask the actual functioning of an individual; laboratory-based motion analysis systems found differences in function between surgical intervention groups whereas no difference was found using clinical outcome scales $(2,4,5)$. Although there is some dispute, studies have shown that clinical outcome scales are valid, reliable and responsive (4,6-8). Maximal performance tests, such as the Timed Up and Go Test, have been shown to be a valid addition to the portfolio of clinical outcome scales, since each assessment method covers a different dimension of function; function is assessed objectively as well as subjectively. However, the outcomes of these maximal performance tests (e.g. time taken to perform a task, distance walked in 6 minutes) have been shown to be too simplistic as they provide no information about the quality of performance. In addition, maximal performance tests are more of a fitness test (e.g. perform this task as fast/well as you can) rather than being related to activities of daily life $(5,9)$. Because walking is one of the basic activities of daily life, gait analysis is frequently used as a measure of function in daily living. To overcome the subjectivity and pain dominance of clinical outcome scales gait analysis has been recommended as an additional means of evaluating patients (10). Currently, in clinical practice analysis of gait is done mainly by visual observation because this is the easiest and fastest method of assessment $(2,11)$. However, this method has been shown to be inadequate for accurate assessment of gait (12). Laboratory-based motion analysis systems, such as 3D-motion analysis, provide accurate gait analysis, and have been used to evaluate the success of surgical intervention and to monitor the progress of pathologies $(2,13)$. However, the complexity, high costs and practical set-up requirements of these techniques (lab-dependency, wired equipment, small and short walkway) make them less suitable for routine clinical use. As an alternative, a portable and easy and quick to use acceleration-based gait analysis (AGA) test has become available. Recently, the AGA-test has proved reliable and reproducible in assessing gait in healthy subjects in normal hospital settings (14). It has also been 
shown to be sensitive enough to detect small changes in gait evoked by simulated functional knee limitations in healthy subjects while performing a walking test (15). These characteristics of AGA make it suitable for routine clinical use, allowing the objective assessment of function in many subjects in a relatively short time period, outside standard laboratory settings, the latter providing conditions more closely resembling daily life. Moreover, using AGA assessments the function of patients can be monitored frequently and objectively during recovery, providing more detailed insight into their functional progress and remaining impairments. Deviations from normal walking patterns can be identified early allowing therapy adjustments which may ultimately improve rehabilitation.

The current study investigated the suitability of AGA for monitoring function in orthopaedic patients with symptomatic gonarthrosis listed for TKA. First, the ability of AGA to distinguish patients from matched healthy subjects was investigated. Second, the sensitivity of AGA to gait changes was investigated by studying the recovery of gait in patients following total knee implantation. Third, correlations between AGA parameters and clinical outcome scales were calculated to investigate whether AGA provides additional information about function beyond the clinical scales.

\section{MethodS}

\section{Patients}

In a case-control study, a cohort of 24 patients $(11 \mathrm{M} / 13 \mathrm{~F}$, mean age $70 \pm 8$ years, weight $85 \pm 15 \mathrm{~kg}$, height $1.69 \pm 0.09 \mathrm{~m}, \mathrm{BMl} 30.0 \pm 8.0 \mathrm{~kg} / \mathrm{m}^{2}$ ) suffering symptomatic gonarthrosis of the knee and receiving unilateral TKA, and 24 age-, gender-, height- and weight-matched healthy subjects ( $11 \mathrm{M} / 13 \mathrm{~F}$, mean age $70 \pm 8$ years, weight $79 \pm 16 \mathrm{~kg}$, height $1.71 \pm 0.09 \mathrm{~m}, \mathrm{BMI} 27.29 \pm 4.0 \mathrm{~kg} / \mathrm{m}^{2}$ ) were included to investigate whether AGA can differentiate pre-operative TKA patients from healthy controls. All patients received the Uncemented Scorpio total knee system (Stryker) using a parapatellar or subvastus approach. The operation was performed by two experienced surgeons. After-treatment was similar for all patients following the same rehabilitation protocol. Full weight-bearing and active exercises were commenced the first day after surgery. The average hospital stay was 4.5 days.

In a prospective cohort study including a subpopulation of the patient group as convenience sample, 12 patients $(6 \mathrm{M} / 6 \mathrm{~F}$, mean age $67 \pm 10$ years, height $1.71 \pm$ $0.08 \mathrm{~m}$, weight $84 \pm 15 \mathrm{~kg}, 29 \pm 6 \mathrm{~kg} / \mathrm{m}^{2}$ ) were followed for 3 months, including surgical intervention. Measurements were taken pre-operatively, 2 weeks, 6 weeks and finally 3 months following surgery. An age-, gender-, height- and weightmatched subpopulation of the healthy control group $(6 \mathrm{M} / 6 \mathrm{~F}$, mean age $67 \pm 9$ 
years, height $1.72 \pm 0.08 \mathrm{~m}$, weight $84 \pm 16 \mathrm{~kg}, \mathrm{BMI} 28 \pm 4 \mathrm{~kg} / \mathrm{m}^{2}$ ) was included as a control group serving as reference with which to compare the 3 months postoperative values. The study was approved by the local ethical committee; all participants gave their informed consent.

\section{Clinical outcome scales}

Clinical outcome scales, the Knee Society Score (knee and function score), the Womac (pain, function and stiffness score), the Pain Disability Index (PDI) and the Visual Analogue Score used as a pain scale (VAS) were measured pre-operatively, at 2 weeks, 6 weeks and 3 months post-operative in the patient group. These scales were not applied to the control group, as healthy subjects are assumed to score maximum on these clinical outcome scales (7).

\section{Acceleration-based gait analysis (AGA)}

Gait was analyzed using a triaxial accelerometer (Minimod, McRobertsBV; dimensions $62 \times 41 \times 18 \mathrm{~mm}, 53 \mathrm{~g}, \mathrm{f}=100 \mathrm{~Hz}$ (16)) which was fixed to the skin at the level of the sacrum using adhesive tape (Fig. 1). Subjects walked a $20 \mathrm{~m}$ straight distance in a hospital corridor six times at their preferred speed. All subjects walked without the use of walking aids. Gait parameters were calculated by proprietary, non-disclosed algorithms of the manufacturer based on the algorithms by Zijlstra and Brandes et al. (17). The basic gait parameters evaluated were step length, step frequency and walking speed. Other clinically relevant parameters included were vertical displacement of the Centre of Mass (CoM), and gait irregularity, which indicates the variability in successive steps of the same leg. The irregularity was calculated as the average of the standard deviation of the left step time and the standard deviation of the right step time. The left-right asymmetry was calculated afterwards as the difference between the average left and right step time divided by the bilateral average (left and right step times determined by the proprietary, non-disclosed algorithms of the manufacturer). Averages of the gait parameters over the six trials were used for analysis. To minimize the inter-subject variation in gait between the patients and the healthy group, the step length, step frequency, speed and vertical displacement of the body were scaled for leg length (based on body height) according to Hof (18).

\section{STATISTICAL ANALYSIS}

After testing the data for normality, the ability of AGA to distinguish patients with symptomatic gonarthrosis listed for TKA from matched healthy subjects was evaluated by comparing AGA parameters between both groups with the independent t-test or the Mann-Whitney U-test. Receiver operating characteristic 

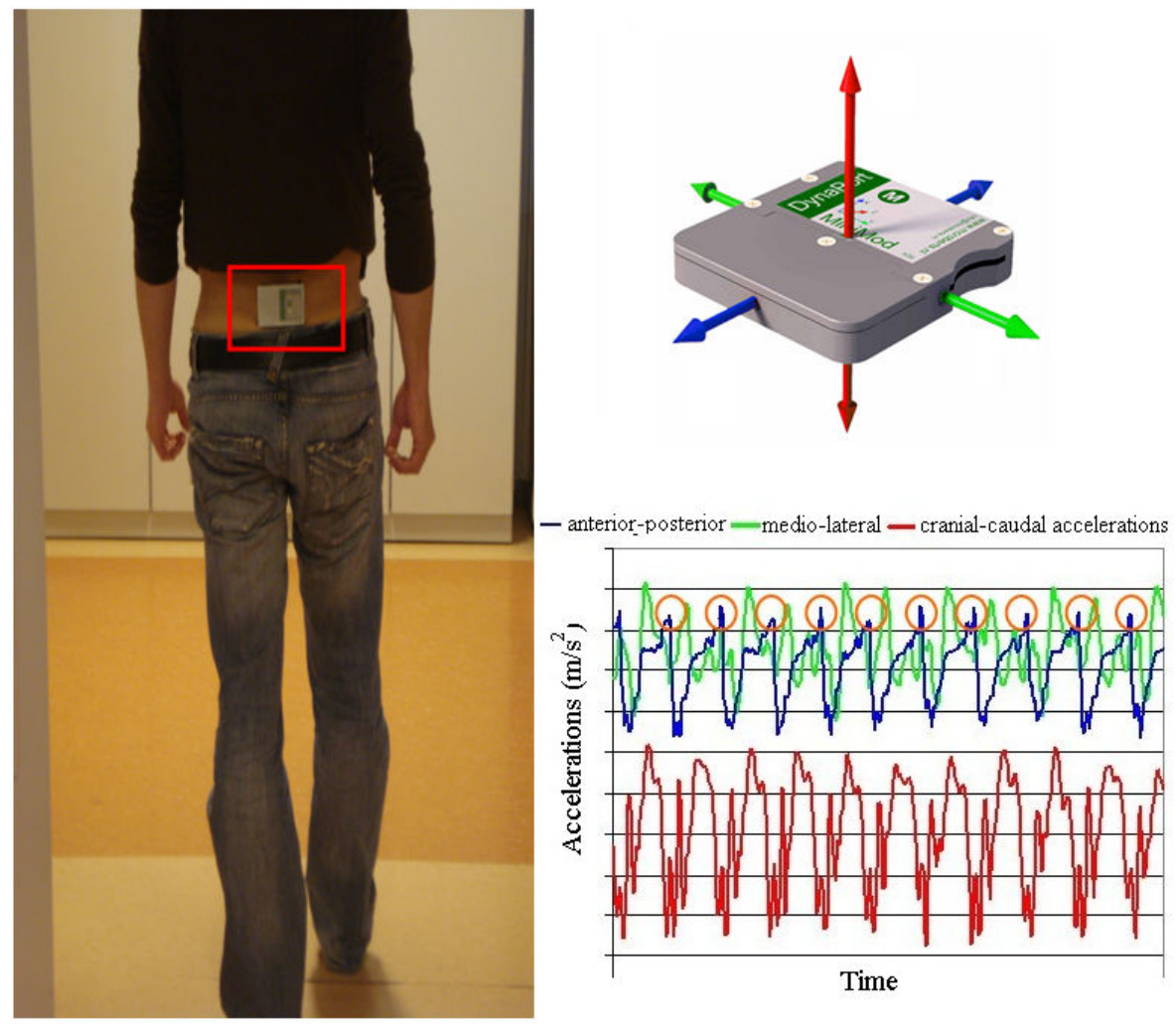

Fig. 1. The experimental set up. A triaxial accelerometer (right top) is attached at the level of the sacrum using double sided tape while walking (left). Peak detection algorithms are used to recognize steps from the anterior-posterior acceleration signal (right bottom).

(ROC) curves were calculated. The area under the curve (AUC) of the ROC, and sensitivity and specificity values were determined to set threshold values distinguishing pre-operative TKA patients from healthy subjects. The closer values of AUC are to $100 \%$, the better the discriminative power between healthy and pathological gait. Optimal thresholds for gait parameters were determined based on the best balance of sensitivity and specificity. Changes over time in AGA parameters and clinical outcome scales were evaluated by repeated measurement ANOVA using LSD for multiple comparisons. The independent t-test or the MannWhitney U-test was used to compare gait of 3 month post-operative patients with the healthy control group. Pearson correlations were used to test the correlations between clinical outcome scales and AGA parameters. All statistical operations were performed using SPSS version 15.0 and differences were considered significant if $\mathrm{p}$-values were less than 0.05 . 


\section{RESULTS}

AGA parameters: discriminating patients from healthy participants

Patients indicated for TKA differed significantly in gait from healthy subjects. Preoperative TKA patients walked significantly more slowly $(1.02 \pm 0.19 \mathrm{~m} / \mathrm{s}$ vs. $1.33 \pm$ $0.16 \mathrm{~m} / \mathrm{s}, \mathrm{p}<0.00)$, with smaller steps $(0.60 \pm 0.08 \mathrm{~m}$ vs. $0.69 \pm 0.08 \mathrm{~m}, \mathrm{p}<0.00)$, lower step frequency (1.69 \pm 0.18 steps/s vs.1.92 \pm 0.17 steps $/ \mathrm{s}, \mathrm{p}<0.00)$, higher vertical displacements $(4.89 \pm 1.43 \mathrm{~cm}$ vs. $4.13 \pm 8.78 \mathrm{~cm}, \mathrm{p}=0.03)$, higher irregularity $(2.9 \pm 1.4 \%$ vs. $2.3 \pm 1.9 \%, p=0.03)$ and higher asymmetry $(5.8 \pm 4.6 \%$ vs. $3.4 \pm 3.2 \%, p=0.05)$. Leg length corrected gait parameters showed similar differences (Table 1). Based on the ROC curves, the following gait parameters showed good discriminative power in delineating pathological from healthy performance; walking speed, step frequency and step length (range AUC 83-89\%). Walking speed, step frequency and step length showed high sensitivity (range 83 96\%) and specificity (range 67 - 71\%). Lower AUC (range 15 - 35\%) results were found for vertical displacement, asymmetry, irregularity and step time ( Table 2). Similar results were found for the height-corrected gait parameters.

\section{Post-surgery recovery patterns}

In the first weeks after TKA, AGA parameters showed impaired function compared to healthy, and even pre-op, status. At 2 weeks following surgery, significant reductions of $22 \%$ in step frequency, $31 \%$ in walking speed and $13 \%$ in step length compared to baseline were recorded. Two weeks after surgery, vertical displacement, step time and asymmetry showed increases of 101\%, 29\% and 91\% respectively compared to the pre-operative assessment (Table 3 ). In contrast, the VAS, KSS (function and knee score) and PDI scores were similar 2 weeks after surgery compared to pre-operative. With Womac (total score, pain and function subscore), the score was significantly better (range 33 - 39\%) at 2 weeks postoperative compared to pre-operative. Six weeks following surgery, AGA parameters (except asymmetry) and clinical outcome scales (except PDI) were significantly

Table 1: Comparison of the leg length corrected gait parameters between pre- and 3 months postoperative patients and age-matched healthy subjects.

\begin{tabular}{llllll}
\hline & & \multicolumn{2}{c}{ Leg length corrected parameters } \\
& & Step frequency & Speed & Step length & Vert. displ. \\
\hline $\mathrm{n} \mathrm{24/24}$ & pre op & $0.50 \pm 0.05$ & $0.36 \pm 0.07$ & $0.71 \pm 0.08$ & $0.06 \pm 0.02$ \\
& matched healthy & $0.57 \pm 0.04$ & $0.46 \pm 0.06$ & $0.81 \pm 0.07$ & $0.05 \pm 0.01$ \\
& p-value & $0.00^{*}$ & $0.00^{*}$ & $0.00^{*}$ & $0.04^{*}$ \\
\hline $\mathrm{n} \mathrm{12/12}$ & 3m post-op & $0.52 \pm 0.03$ & $0.40 \pm 0.05$ & $0.76 \pm 0.08$ & $0.053 \pm 0.01$ \\
& matched healthy & $0.57 \pm 0.04$ & $0.47 \pm 0.05$ & $0.84 \pm 0.07$ & $0.051 \pm 0.01$ \\
& p-value & $0.01^{*}$ & $0.00^{*}$ & $0.03^{*}$ & 0.41 \\
\hline
\end{tabular}

Averages $\pm \mathrm{SD},{ }^{*} \mathrm{p}<0.05 ; \quad$ Vert. displ.: vertical displacement of CoM 
Table 2: The area under the curve (AUC) of the ROC, sensitivity and specificity values with their corresponding threshold values distinguishing pre operative TKA patients from healthy subjects.

\begin{tabular}{lcccc}
\hline & AUC (\%) & Sensitivity (\%) & Specificity (\%) & Threshold \\
\hline Speed (m/s) & 89 & 96 & 71 & 1.11 \\
Step frequency (steps/s) & 84 & 88 & 67 & 1.74 \\
Step length (m) & 83 & 83 & 71 & 0.60 \\
Step time (s) & 15 & 54 & 13 & 0.53 \\
Vertical displacement (m) & 34 & 58 & 29 & 3.90 \\
Asymmetry (\%) & 35 & 58 & 25 & 2.02 \\
Irregularity (\%) & 32 & 54 & 42 & 1.95 \\
\hline
\end{tabular}

Table 3: Averages \pm SD for clinical outcome scales and gait parameters at different follow up times.

\begin{tabular}{lccccccc}
\hline & \multicolumn{7}{c}{ Follow Up time } \\
\cline { 2 - 8 } $\mathbf{n = 1 2}$ & pre & 2 weeks & p-value & 6 weeks & p-value & 3 months & p-value \\
\hline VAS & $3.4 \pm 2.7$ & $3.9 \pm 2.5$ & 0.65 & $2.0 \pm 1.7$ & 0.14 & $1.9 \pm 2.3$ & 0.13 \\
KSS Knee & $56.8 \pm 15.4$ & $57.5 \pm 15.2$ & 0.89 & $73.8 \pm 17.7$ & $0.02^{*}$ & $81.3 \pm 15.6$ & $0.00^{*}$ \\
KSS Function & $45.8 \pm 14.4$ & $49.9 \pm 9.5$ & 0.49 & $71.0 \pm 13.1$ & $0.01^{*}$ & $82.9 \pm 11.3$ & $0.00^{*}$ \\
Womac Pain & $12.8 \pm 5.2$ & $8.6 \pm 3.5$ & $0.00^{*}$ & $5.9 \pm 3.7$ & $0.01^{*}$ & $5.6 \pm 4.2$ & $0.00^{*}$ \\
Womac Stiffness & $5.2 \pm 1.6$ & $4.8 \pm 1.5$ & 0.45 & $3.7 \pm 2.4$ & 0.10 & $3.6 \pm 2.0$ & 0.06 \\
Womac Function & $42.1 \pm 16.8$ & $25.7 \pm 8.0$ & $0.01^{*}$ & $18.8 \pm 8.7$ & $0.00^{*}$ & $18.6 \pm 11.7$ & $0.00^{*}$ \\
Womac Total & $60.1 \pm 22.7$ & $39.0 \pm 11.7$ & $0.01^{*}$ & $28.4 \pm 13.3$ & $0.00^{*}$ & $27.8 \pm 16.5$ & $0.00^{*}$ \\
PDI & $38.0 \pm 8.5$ & $35.3 \pm 17.8$ & 0.59 & $24.3 \pm 13.7$ & $0.02^{*}$ & $16.1 \pm 15.3$ & $0.00^{*}$ \\
Frequency (Hz) & $1.75 \pm 0.12$ & $1.36 \pm 0.14$ & $0.00^{*}$ & $1.74 \pm 0.18$ & 0.87 & $1.77 \pm 0.12$ & 0.58 \\
Step time (s) & $0.58 \pm 0.04$ & $0.75 \pm 0.08$ & $0.00^{*}$ & $0.58 \pm 0.06$ & 0.90 & $0.57 \pm 0.04$ & 0.39 \\
Irregularity (\%) & $2.48 \pm 1.12$ & $3.42 \pm 1.25$ & 0.08 & $2.36 \pm 0.94$ & 0.71 & $2.24 \pm 0.90$ & 0.24 \\
Step length (m) & $0.62 \pm 0.06$ & $0.54 \pm 0.14$ & $0.048^{*}$ & $0.63 \pm 0.07$ & 0.57 & $0.65 \pm 0.08$ & 0.26 \\
Speed (m/s) & $1.07 \pm 0.14$ & $0.74 \pm 0.24$ & $0.00^{*}$ & $1.09 \pm 0.14$ & 0.83 & $1.14 \pm 0.16$ & 0.29 \\
Vert. displ. (cm) & $4.34 \pm 0.99$ & $8.73 \pm 2.97$ & $0.00^{*}$ & $4.50 \pm 1.48$ & 0.62 & $4.44 \pm 1.11$ & 0.69 \\
Asymmetry (\%) & $5.61 \pm 4.99$ & $10.71 \pm 6.46$ & $0.04^{*}$ & $5.62 \pm 4.65$ & 0.97 & $6.47 \pm 4.50$ & 0.56 \\
\hline
\end{tabular}

* significant difference with regard to pre-operative ( $p<0.05)$; Vert. displ.: vertical displacement of CoM

improved compared to the 2 weeks post-operative assessment, showing improvements ranging between $17 \%$ and $48 \%$ for gait parameters and between $23 \%$ and $49 \%$ for clinical outcome scales. At 6 weeks following surgery, gait parameters just reached pre-operative levels, whereas KSS (knee and function), Womac (pain, function, total) and PDI significantly exceeded pre-operative levels. Clinical outcome scales continued to improve between the 6 weeks and 3 month post-operative assessments, showing significantly better scores 3 months postoperative compared to pre-operative. AGA parameters only started to improve from 6 weeks post-operatively, but no significant improvement compared to preoperative was found 3 months following surgery. In addition, the functional status at 3 months post-operative as measured by AGA was still well below healthy levels (Table 1). Three months post-operative patients still walked significantly more slowly $(1.14 \pm 0.16 \mathrm{~m} / \mathrm{s}$ vs. $1.38 \pm 0.13 \mathrm{~m} / \mathrm{s}, \mathrm{p}<0.00)$, with significantly lower step frequency $(1.77 \pm 0.12$ steps/s vs. $1.92 \pm 0.17$ steps $/ \mathrm{s}, \mathrm{p}=0.02)$, smaller step length $(0.65 \pm 0.08 \mathrm{~m}$ vs. $0.72 \pm 0.07 \mathrm{~m}, \mathrm{p}=0.02)$, significantly higher step times $(0.57 \pm$ 0.04 s vs. $0.53 \pm 0.04 \mathrm{~s}, \mathrm{p}=0.02)$ and asymmetry $(6.5 \pm 4.5 \%$ vs. $2.3 \pm 2.0 \%, p=0.01)$ compared to the healthy age-matched group. The irregularity $(2.2 \pm 0.9 \%$ vs. $2.1 \pm$ 
Table 4: Correlations between AGA parameters and clinical outcome scales.

\section{CLINICAL OUTCOME SCALES}

\begin{tabular}{|c|c|c|c|c|c|c|c|c|}
\hline & & VAS & $\begin{array}{l}\text { KSS } \\
\text { knee }\end{array}$ & $\begin{array}{l}\text { KSS } \\
\text { Function }\end{array}$ & $\begin{array}{l}\text { Womac } \\
\text { pain }\end{array}$ & $\begin{array}{l}\text { Womac } \\
\text { stiffness }\end{array}$ & $\begin{array}{l}\text { Womac } \\
\text { function }\end{array}$ & PDI \\
\hline \multirow{7}{*}{ 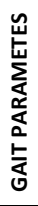 } & Step frequency $(\mathrm{Hz})$ & -0.20 & 0.22 & $-0.34^{*}$ & -0.03 & -0.21 & -0.09 & $-0.29 *$ \\
\hline & Step time (s) & 0.21 & -0.22 & $-0.31 *$ & 0.02 & 0.17 & 0.09 & $0.30 *$ \\
\hline & Irregularity (\%) & 0.11 & -0.14 & $-0.30 *$ & 0.07 & 0.19 & 0.09 & $0.26^{*}$ \\
\hline & Step length (m) & -0.16 & -0.13 & $0.30 *$ & 0.00 & -0.12 & -0.08 & -0.18 \\
\hline & Walk speed $(\mathrm{m} / \mathrm{s})$ & $-0.25^{*}$ & -0.18 & $0.41^{*}$ & -0.02 & -0.2 & -0.1 & $-0.26^{*}$ \\
\hline & Vert. displ (cm) & $0.24^{*}$ & $-0.39 *$ & $-0.34 *$ & 0.09 & 0.19 & 0.05 & 0.29 \\
\hline & Asymmetry (\%) & -0.08 & -0.02 & -0.12 & 0.05 & 0.15 & 0.02 & 0.06 \\
\hline
\end{tabular}

Correlation coefficients ( $\left.{ }^{*} \mathrm{p}<0.05\right)$; Vert. displ : vertical displacement of CoM

$1.7 \%, p=0.38)$ and vertical displacement $(4.44 \pm 1.11 \mathrm{~cm}$ vs. $4.27+0.84 \mathrm{~cm}, \mathrm{p}=$ 0.068 ) were slightly higher in the patient group; however, these differences were not significant. Similar differences between 3 month post-operative patients and healthy subjects were found using height scaled gait parameters (Table 1).

\section{Correlations}

Few, and only weak, correlations (range $r=0.23-0.41$ ) were found between several AGA parameters and clinical outcome scales (KSS, PDI, VAS). The KSS function subscore correlated significantly with every gait parameter except asymmetry, showing correlation coefficients ranging from -0.23 to 0.41 . Womac (sub) score correlated with none of the gait parameters (Table 4).

\section{Discussion}

The current study investigated whether acceleration-based gait analysis (AGA) is suitable for assessing the walking performance of orthopaedic patients. Specifically it evaluated whether AGA is (a) able to differentiate pathological from healthy gait, (b) sensitive to gait changes when monitoring TKA patients during recovery and (c) able to provide additional information beyond the conventional clinical outcome scales.

AGA parameters: discriminating patients from healthy participants

AGA was able to differentiate gait of patients with symptomatic gonarthrosis listed for TKA from healthy gait, showing high sensitivity and specificity. For instance, a threshold level of $60 \mathrm{~cm}$ and lower for the step length showed a sensitivity of $83 \%$ in identifying patients listed for TKA, while only two of the 24 healthy subjects had a step length smaller than $60 \mathrm{~cm}$. The assessment of walking speed showed the best discriminative power in distinguishing pathological from healthy performance achieving an AUC of $89 \%$ (threshold level: $1.11 \mathrm{~m} / \mathrm{s}=4.0 \mathrm{~km} / \mathrm{h}$ ). Actually, AGA is not necessary to determine walking speed, because it can also be measured using a 
stopwatch. However, for measurements over longer time periods, for instance in daily life conditions, the use of a stopwatch is impractical. In addition AGA produces several gait parameters simultaneously, allowing extended and detailed insight into the function of subjects. These parameters (e.g. step length, frequency) also showed high discriminative power suggesting that combining thresholds for several gait parameters could further improve the discriminative power of AGA. The high sensitivity and specificity values of AGA indicate that accelerometers are powerful tools for identifying deviations in gait shown in patients with symptomatic gonarthrosis listed for TKA. However, even after scaling for body size, there was a range of overlap in gait between the healthy and patient groups, which probably reflects the relatively wide range of different gait styles encountered in the population. Clinical outcome scales assume that healthy subjects score almost excellent results (e.g. KSS between 80 and 100), while worse scores are expected for patients, which assumes that there is no overlap between healthy subjects and patients. However, clinical outcome scales are not primarily intended as a diagnostic tool, but serve mainly to measure outcomes when evaluating patients $(4,6-8,19,20)$. AGA, in contrast, can probably also serve as diagnostic tool. However, further investigation is required into the diagnostic power of AGA in differentiating between pathologies.

Patients listed for TKA showed significantly impaired gait parameters as measured by AGA. The slower walking speed of TKA patients, a combination of significantly lower step frequency and shorter step length, was comparable to previously observed findings using laboratory-based methods (21). Also the absolute values of the gait data produced by AGA were quite similar to other validated methods reported in the literature. For instance, a walking speed of $1.07 \mathrm{~m} / \mathrm{s}$, a step frequency of 1.66 steps/s and a step length of $0.63 \mathrm{~m}$ were found in osteoarthritis patients listed for surgery (2). These values correspond to the $1.02 \mathrm{~m} / \mathrm{s}, 1.69$ steps/s and $0.60 \mathrm{~m}$ found in the current study.

In this study gait variability was determined by step irregularity and asymmetry. However, variability during gait can be expressed using several definitions, making comparison with other studies impossible. For instance Yakhdani et al. expressed variability as the mean stride-to-stride standard deviation of angular velocity of sagittal knee movements (22), while the current study uses irregularity to express gait variability as the average standard deviation of the left and right step time. Increased irregularity was found in patients indicating that the walk of healthy controls is more regular, while more variation between steps is found in the patient group. This gait pattern may be the result of a compensation strategy for limited function, or it may be due to the instability experienced by these patients (23).

Patients showed higher displacements in a vertical direction than healthy controls. The higher up-down movement in patients while walking may be caused by the symptomatic knee, which is frequently indicated by a limited knee flexion during 
the stance and swing phase of gait $(13,24,25)$. The increased asymmetry found in pre-operative patients corresponds with previous studies which indicated that patients unload their affected leg as much as possible inducing asymmetric leg behaviour $(2,26)$. In four cases extremely high asymmetry values were obtained (10 - 18\%). This may be due to the fact that the sample frequency of the accelerometer is too low to accurately identify the peaks which are used to calculate the asymmetry (14). Because doubts exist about the correctness of these four high asymmetry values, the analysis was also performed without these values. However, no difference in the overall results was found.

\section{Post-surgery recovery patterns}

Clinical outcome scales and AGA parameters displayed different post-operative recovery profiles. Immediately after surgery, clinical outcome scales recorded an improvement in function over pre-op levels, while according to the AGA parameters function was reduced. Correspondingly, Harato et al. found a reduction in pain 1 week after TKA, while objective knee function measurements (vertical knee force and knee flexion angle of involved leg) only improved from postoperative day 16-17 (27). Six weeks following surgery, scores on clinical outcome scales were significantly better than pre-operative, while AGA parameters only reached pre-operative levels. This faster recovery in clinical outcome scales may be due to the subjectivity of the scales which are pain dominated and satisfaction based $(6,28)$. Pain-free movement, together with the ability to perform a more demanding activity again (e.g. stair climbing) after a certain period of incapacity, results in high patient satisfaction, which is reflected in high scores on clinical outcome scales. This indicates the importance of pain relief for patients shortly following surgery. The considerable changes in gait immediately following intervention, like the significant reduction in walking speed and step length, may be due to adaptations by patients to regain stable and safe gait post-operatively. Such changes in gait are also observed in older subjects compensating for age-related impairments in order to regain stability (29).

AGA parameters first started to improve at 6 weeks post-operative, showing improved (not significantly) gait parameters 3 months post-operative compared to pre-operative. The improvements in gait shown during the first 3 months following surgery were similar to the improvements observed in the study by Börjesson et al. who used force plate measurements to analyze the gait of 40 patients suffering knee osteoarthritis. Börjesson's study indicated that the average walking speed increased by $8 \%$ (1.07 to $1.16 \mathrm{~m} / \mathrm{s}$ ) from pre-operative to 3 months after surgery, corresponding to the $7 \%(1.07$ to $1.14 \mathrm{~m} / \mathrm{s})$ improvement measured in the current study (2). The fact that the changes observed were similar in magnitude to those reported by Börjesson (2), but not significant, suggests that the power of the current study is relatively low with respect to these clinically important changes. 
However, the fact that similar changes were observed shows the applicability of AGA in monitoring patients during recovery.

The clinical outcome scales continued to improve between the 6 weeks and 3 month follow ups. Spencer et al. (30) reported that clinical outcome scores did not change between 3 months and 2 years post-operative. This suggests that clinical outcome scales more or less reach a plateau, which is probably due to fast recovery immediately after surgery. This ceiling effect in clinical outcome scales, when gait has just started to improve, was also indicated by the fact that the KSS knee and function scores improved by $43 \%$ (and $81 \%$ respectively) from pre-operative to 3 months post-operative, suggesting that the KSS ceiling is too low. Step length (and walking speed) improved by $5 \%$ (and 7\%, respectively) from pre-operative to 3 month post-operative, remaining respectively $10 \%$ (and 15\%) below healthy values. Three months after surgery, AGA parameters remained significantly below healthy levels. This has previously been shown using laboratory-based methods (13). Studies indicated that it is mainly the quadriceps strength of the non-affected leg that is involved in improvements in functional performance during the first 3 months, while the relation between functional performance and quadriceps strength of the affected leg increased after 3 months (10). This suggests that function will improve further beyond 3 months post-operative, and therefore that rehabilitation and physiotherapy may need to continue for longer than 3 months $(10,31)$. However, longer follow up studies showed that TKA patients even at 1 or 2 years post-operative still walked significantly more slowly than their matched controls (13). It is also suggested that patients often fail to reach healthy levels, even years after surgery (32). It is therefore unclear whether the best adapted gait pattern of TKA patients is comparable to healthy levels. Further research is needed to investigate the optimal gait pattern of patients following TKA.

\section{Correlations}

In line with previous studies, different recovery profiles were found with AGA and clinical outcome scales, suggesting the usefulness of combining both evaluation methods when evaluating post-operative recovery. Also the lack of, or only weak, correlations observed between the clinical outcome scales and AGA parameters indicate that each assessment measures different aspects of functioning as was previously shown $(9,33)$. For instance, Hoeymans et al. found low correlations between self-reported disabilities in the activities of daily life and walking speed (range $r=0.13-0.33$ ) (33). Moderate correlations were found between the Study Short Form Healthy Survey function score and a maximal treadmill walking test $(r=$ 0.43) (9). Also Fuchs et al. showed only weak correlations between locomotion criteria and the Hospital for Special Surgery Score, KSS and VAS score (34). Most correlations were found between the KSS function subscore and AGA parameters, suggesting that among conventional scores the function score of KSS gives the most 
objective measure of a patient's functional state. However, this may be due to the fact that questions on the KSS function score are only about walking distance, stair climbing ability and use of a walking aid, which correspond to the AGA-test, while the Womac subscales consist of more varied items about ADL, and the VAS and PDI scales only measure pain (7). In addition, Womac (sub) scores do not seem to capture objective functional changes as measured by AGA suggesting that Womac (sub) scores lack a functional aspect. Correspondingly, Witvrouw et al. indicated that the outcome of Womac is strongly influenced by factors other than quantitative parameters of the lower extremity, which were measured using an acceleration-based Dynaport Classic Test which consists of the performance of ADL activities (5). The fact that AGA and clinical outcome scales show differences in measuring recovery rates demonstrates the additional value of using AGA for monitoring patients during recovery in clinical practice.

\section{Clinical implications}

It has been reported that the new generation of increasingly younger TKA patients are satisfied with the extent of pain relief after surgery, but their expectations about physical function remain unfulfilled (3). This patient demand for higher postoperative functional levels shows the rising importance of objective assessment of function in clinical practice (3). To reach optimal functional levels, post-operative rehabilitation programs could be improved by frequent and objective functional assessment. AGA allows the frequent monitoring of patients, providing additional objective information about a patient's function beyond the clinical scales. This suggests the suitability of AGA in providing feedback to the patient, therapist and clinical researcher as part of routine clinical practice. By routinely evaluating the effect of therapy and intervention on recovery, therapy can be adjusted to an individual's needs, which could ultimately optimize the recovery process. However further research is needed to investigate this.

\section{CONCLUSION}

The current study showed the clinical relevance of AGA as a functional assessment tool to identify and routinely monitor patients with symptomatic gonarthrosis in clinical practice. 


\section{REFERENCES}

1. Ritter MA, Wing JT, Berend ME, Davis KE, Meding JB. The clinical effect of gender on outcome of total knee arthroplasty. J Arthroplasty 2008;23(3):331-6.

2. Börjesson $M$, Weidenhielm L, Mattsson E, Olsson E. Gait and clinical measurements in patients with knee osteoarthritis after surgery: a prospective 5-year follow up study. The Knee 2005;12:121-7.

3. Nilsdotter AK, Toksvig-Larsen S, Roos EM. Knee arthroplasty: Are patients' expectations fulfilled? Acta Orthop 2009;80(1):55-61.

4. Terwee CB, van der Slikke RMA, van Lummel RC, Benink RJ, Meijers WGH, de Vet HCW. Selfreported physical functioning was more influenced by pain than performance-based physical functioning in knee-osteoarthritis patients. J Clin Epidemiol 2006;59:724-31.

5. Witvrouw E, Victor J, Bellemans J, Rock B, Van Lummel R, van der Slikke R. A correlation study of objective functionality and WOMAC in total knee arthroplasty. Knee Surg Sports Traumatol Arthrosc 2002;10(6):347-51.

6. Liebensteiner MC, Herten A, MGstoettner AH, Thaler M, Krismer M, Back CM. Correlation between objective gait parameters and subjective score measurements before and after total knee arthroplasty. Knee 2008;15:461-6.

7. Lingard EA, Katz JN, Wright RJ, Wright EA, Sledge CB, The Kinemax Outcomes Groups. Validity and responsiveness of the knee society clinical rating system in comparison with the SF-36 and WOMAC. J Bone Joint Surg 2001;83-A(12):1856-64.

8. Stratford PW, Kennedy DM. Does parallel item content on WOMAC's pain and function subscales limit its ability to detect change in functional state? BMC Musculoskelet Disord 2004;5:17-25.

9. Wittink H, Rogers W, Sukiennik A, Carr DB. Physical functioning: self-report and performance measures are related but distinct. Spine 2003;28:2407-13.

10. Yoshida Y, Mizner RL, Ramsey DK, Snyder-Mackler L. Examining outcomes from total knee arthroplasty and the relationship between quadriceps strength and knee function over time. Clin Biomech 2008;23(3):320-8.

11. Ishii Y, Terajima K, Koga Y, Takahashi HE, Bechtold JE, Gustilo R. Gait analysis after total knee arthroplasty. Comparison of posterior cruciate retention and substitution. J Orthop Sci 1998;3(6):310-7.

12. Saleh M, Murdoch G. In defence of gait analysis. Observation and measurement in gait assessment. J Bone Joint Surg 1985;67-B(2):237-40.

13. Benedetti MG, Catani F, Bilotta TW, Marcacci M, Mariani E, Giannini S. Muscle activation pattern and gait biomechanics after total knee replacement. Clin Biomech 2003;28(9):871-6.

14. Senden R, Grimm B, Heyligers IC, Savelberg HHCM, Meijer K. An acceleration-based gait test for healthy subjects: Reliability and reference data. Gait Posture 2009;30(2):192-6.

15. Grimm B, Vanderherst T, Munch C. Heyligers I.C. Application feasibility of accelerometer based gait analysis in clinical orthopaedics. J Biomech 2006;39: S110.

16. Brandes M, Zijlstra W, Heikens S, Lummel van R. Accelerometry based assessment of gait parameters in children. Gait Posture 2006;24:482-6.

17. Zijlstra W, Hof AL. Assessment of spatio-temporal gait parameters from trunk accelerations during human walking. Gait Posture 2003;18:1-20.

18. Hof AL. Scaling gait data to body size. Gait Posture 1996;4:222-3.

19. Insall J. Rating systems for total knee replacement. The Knee 2002;9(4):261-6.

20. Kreibich DN, Vaz M, Bourne RB, Rorabeck $\mathrm{CH}$, Kim P, Hardie R, et al. What is the best way of assessing outcome after total knee replacement? Clin Orthop Relat Res 1996;331:221-5.

21. Ivarsson I, Gillquist J. Rehabilitation after high tibial osteotomy and unicompartmental arthroplasty. A comparative study. Clin Orthop 1991;266:139-44.

22. Yakhdani HRF, Bafghi HA, Meijer OG, Bruijn SM, Dikk van den N, Stibbe AB, et al. Stability and variability of knee kinematics during gait in knee osteoarthritis before and after replacement surgery. Clin Biomech 2010;25(3):230-6.

23. Fitzgerald GK, Piva SR, Irrgang JJ. Reports of joint instability in knee osteoarthritis: its prevalence and relationship to physical function. Arthritis Rheum 2004;51: 941-6. 
24. Finch E, Walch M, Thomas SG, Woodhouse L. Functional ability perceived by individuals following total knee arthroplasty compared to age-matched individuals without knee disability. J Orthop Sports Phys Ther 1998;27(4):255-63.

25. Milner CE. Is gait normal after total knee arthroplasty? Systematic review of the literature. J Orthop Sci 2009;14(1):114-20.

26. Mizner RL, Snyder-Mackler L. Altered loading during walking and sit-to-stand is affected by quadriceps weakness after total knee arthroplasty. J Orthop Res 2005;23:1083-90.

27. Harato K, Otani T, Nakayama N, Watarai H, Wada M, Yoshimine F. When does post-operative standing function after total knee arthroplasty improve beyond pre-operative level of function? Knee 2009;16:112-25.

28. Mandeville D, Osternig LR, Chou LS. The effect of total knee replacement surgery on gait stability. Gait Posture 2008;27(1):103-9.

29. Winter DA, Patla AE, Frank JS, Walt SE. Biomechanical walking pattern changes in the fit and healthy elderly. Phys Ther 1990;79(6):340-7.

30. Spencer JM, Chauhan SK, Sloan K, Taylor A, Beaver RJ. Computer navigation versus conventional total knee replacement. No difference in functional results at two years. J Bone Joint Surg $\mathrm{Br}$ 2007;89B(4):477-80.

31. Webster KE, Witter JE, Feller JA. Quantitative gait analysis after medial unicompartmental knee arthroplasty for osteoarthritis. J Arthroplasty 2003;18(6): 751-9.

32. Lee TH, Tsuchida T, Kitahara H, Moriya H. Gait analysis before and after unilateral total knee arthroplasty. Study using a linear regression model of normal controls- women without arthroplasty. J Orthop Sci 1999;4:13-21.

33. Hoeymans N, Feskens EJ, van den Bos GA, Kromhou D. Measuring functional status: cross sectional and longitudinal associations between performance and self-report. J Clin Epidemiol 1996;49:110310.

34. Fuchs S, Genkinger M, Laass H, Rosenbaum D. Results of bicondylar sledge prostheses with special reference to gait pattern. Biomed Tech (Berl) 2001;46: 142-6. 



\section{Chapter 6}

Accelerometry-based gait analysis, an additional objective approach to screen subjects at risk for falling

Senden R, Savelberg HHCM, Grimm B, Heyligers IC, Meijer K.

Gait Posture 2012; 36(2):296-300. 


\section{ABSTRACT}

This study investigated whether the Tinetti scale, as a subjective measure for fall risk, is associated with objectively measured gait characteristics. It is studied whether gait parameters are different for groups that are stratified for fall risk using the Tinetti scale. Moreover, the discriminative power of gait parameters to classify elderly according to the Tinetti scale is investigated. Gait of 50 elderly with a Tinneti $>24$ and 50 elderly with a Tinetti $\leq 24$ was analyzed using accelerationbased gait analysis. Validated algorithms were used to derive spatio-temporal gait parameters, harmonic ratio, inter-stride amplitude variability and root mean square (RMS) from the accelerometer data. Clear differences in gait were found between the groups. All gait parameters correlated with the Tinetti scale ( $r$-range : 0.20 - 0.73). Only walking speed, step length and RMS showed moderate to strong correlations and high discriminative power to classify elderly according to the Tinetti scale. It is concluded that subtle gait changes that have previously been related to fall risk are not captured by the subjective assessment. It is therefore worthwhile to include objective gait assessment in fall risk screening. 


\section{INTRODUCTION}

Falls are the most common accidents in elderly causing serious problems; in addition falls have shown to be the sixth leading cause of death among elderly (1). Numerous factors have been related to fall risk, including changes in gait (1-4). Up to $70 \%$ of the falls in elderly occur during walking. Fletcher et. al. showed that individuals with an impaired mobility were 1.65 times more likely to experience a fall (5). Indeed, gait and balance disturbances are shown to be better predictors for imminent falls than other risk factors like for instance impaired vision (6). Several gait characteristics have been associated with fall risk and fall history, i.e. slow speed, shortened swing phase, increased gait unsteadiness and stride-to-stride variability $(1,3-4,7-8)$.

Various tools are currently applied in clinical practice to quantify fall risk. At this moment, there is no gold standard. Moreover there is no consistency among clinicians regarding the best tool for assessing fall risk (9). Some assessments rely on functional measures like timing a physical performance. These tools are mainly sensitive for elderly with an increased fall risk, who have visually detectable deviations in function (e.g. decreased ambulation) (10). Questionnaire-based checklists evaluating multi-dimensional risk factors such as psychological status (e.g. Physiological Profile Approach (PPA) (11)) showed to be reliable and valid to assess fall risk, but they are not suitable for clinical use where time is limited (11). Fall history has also frequently been employed as indicator for fall risk. This method is clinically feasible, but lacks reliability especially in subjects who are forgetful (12). Other commonly used approaches evaluate functional aspects of postural stability by visually observing functional limitations (e.g. Berg Balance Scale) (9). The Tinetti scale is one of these widely accepted subjective approaches used in clinical settings to assess mobility dysfunctions in elderly (13-15), to assess fall risk in individuals with Parkinson's (16) and Huntington's Disease (17) as well as to predict falls among healthy elderly subjects $(15,18)$. The predictive power of the Tinettibalance subscore for falls is only moderate (Area Under Curve, AUC 0.6), but similar and even higher than the predictive power of functional measures like the Timed Get Up \& Go (AUC 0.6), functional reach (AUC 0.5) and one-leg stance (AUC 0.5) test (15). It could be argued that combining subjective and functional measures can improve fall risk assessment, provided that they capture different aspects of a subjects' fall risk. Since objectively measured gait characteristics (e.g. step length, variability etc.) have been associated with fall risk $(1,3-4,7-8)$ and because gait analysis provides more detailed information about the functional ability of individuals than commonly used functional measures (e.g. Timed Get Up and Go test), objective gait analysis is indicated as an obvious functional measure.

Acceleration-based gait analysis (AGA) has become popular in clinical practice. Accelerometers have been applied to objectively, reliably and reproducibly analyze 
gait in different populations $(2,19-22)$, to identify fear of falling and to differentiate fallers from non-fallers $(5,23)$. In addition the acceleration pattern of the pelvis during walking has been associated with falls risk as measured by the comprehensive PPA (4). Direct relationships between objectively measured gait characteristics as measured by AGA and commonly used subjective scales for fall risk, like the Tinetti scale, are currently missing. It is in addition unknown whether objectively measured gait characteristics are of additional value beyond the commonly used subjective measures for fall risk.

This study investigated whether the Tinetti scale, as a subjective measure for fall risk, is associated with objectively measured gait characteristics related to fall risk. Specifically, it was studied whether AGA-parameters are different for groups that are stratified for fall risk using the Tinetti scale. Moreover, the discriminative power of AGA-parameters to classify elderly according to the Tinetti scale was investigated.

\section{Methods}

Subjects

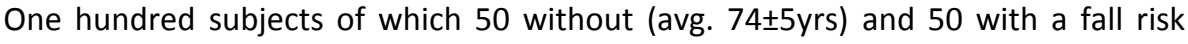
(avg. 79 $\pm 6 y r s)$ were included in this case control study (table 1). The inclusion criteria were: age of 65 years or older, able to walk without assistive devices and no severe cognitive impairments. All subjects were informed about the study and gave written informed consent. Ethics approval was obtained from the local ethical committee.

\section{Fall Risk}

The Tinetti scale was used as an indicator of fall risk (18) which is based on a visual gait and balance assessment. A total score of 24 points or less out of 28 indicates fall risk (18). This test was performed by two researchers trained in the assessment. It took roughly 15-20 minutes to complete the test. The risk group was further divided into a low (Tinetti 19-24/28, n=31) and high (Tinetti <19/28, n=19) risk group.

\section{Test procedure}

Fall history and subject demographics were collected (table 1-2). Fall history was obtained by asking the subjects whether they have fallen in the last six months. Falls were defined as unintentionally coming to rest on the ground. All subjects performed a walking test while a triaxial accelerometer (DAAFb, f $102 \mathrm{~Hz}$ or ETBPegasus, $f 100 \mathrm{~Hz}$, Fig.1) was attached to the level of the sacrum using double sided 

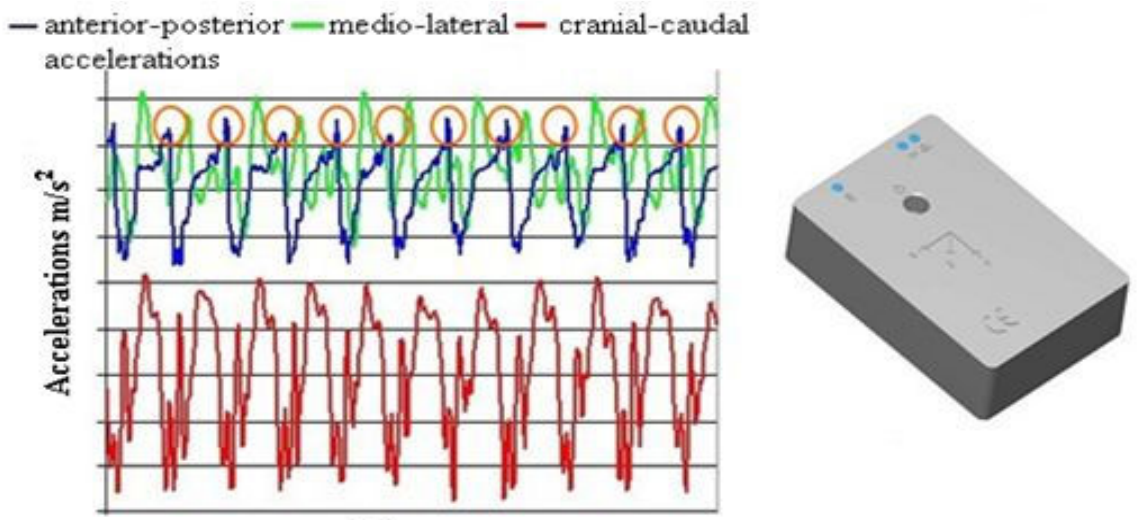

Time

Fig. 1: 3D acceleration signal of the gait pattern (left) measured by the accelerometer (DAAFb, right). Gait parameters are derived using a template peak detection algorithm which relies on the anterior posterior acceleration signal (blue signal). The o represents the peak that is detected to recognise individuals steps.

tape. Subjects walked a $20 \mathrm{~m}$ straight distance in a hospital corridor at preferred speed. Their last step had to take them beyond the $20 \mathrm{~m}$ mark. The additional distance was measured by a ruler to obtain the exact distance walked. After $20 \mathrm{~m}$ subjects turned around and walked back. This procedure was carried out three time, resulting in six walking trials. The duration of the test set-up ( $\pm 1 \mathrm{~min}$ ) and measurement ( $\pm 3 \mathrm{~min}$ ) is more or less 4 minutes. The analysis of the data takes \pm 5 10 minutes. The data analysis relied on semi-automated algorithms requiring some manual intervention (e.g. check for correct peaks, indicate start and stop point) (19).

\section{Gait parameters}

A self-designed template algorithm was applied on the anterior-posterior acceleration signal to identify specific peaks corresponding to foot contact as is shown by Zijlstra. A pilot study, revealed good agreement between our template algorithm and a manual peak detection algorithms according to Zijlstra et al. In this way, steps were recognized and gait parameters were derived (fig.1) (24). Spatiotemporal parameters like number of steps (amount peaks), walking speed (distance/walking time), cadence (number of steps/walking time), step time (time from foot contact to foot contact) and step length (distance/ number of steps) were determined. The step time asymmetry was calculated to indicate differences between left and right leg movements (22). The harmonic ratio, inter-stride amplitude variability and root mean square (RMS), all relying on vertical acceleration signal, were determined as defined and described by Menz et al. (25). The average of gait parameters measured over six trials was used for analysis. 
Table 1: Averages \pm standard deviations of subject characteristics, Tinetti (sub)scores, fall history and AGA-parameters for the groups having a Tinetti $\leq 24$ and the group having a Tinetti $>24$.

\begin{tabular}{lccc}
\hline & $\begin{array}{c}\text { Tinetti } \mathbf{2 4} \\
(\mathbf{n}=\mathbf{5 0})\end{array}$ & $\begin{array}{c}\text { Tinetti } \leq \mathbf{2 4} \\
(\mathbf{n}=\mathbf{5 0})\end{array}$ & p-values \\
\hline Gender (F/M) & $23 / 27$ & $33 / 17$ & 0.07 \\
Age (years) & $74.2 \pm 5.1$ & $78.9 \pm 6.2$ & $<0.01^{*}$ \\
Height (m) & $1.68 \pm 0.09$ & $1.67 \pm 0.11$ & 0.51 \\
Weight (kg) & $72.3 \pm 12.7$ & $70.3 \pm 13.5$ & 0.44 \\
BMI (kg/m) & $25.47 \pm 3.76$ & $25.10 \pm 4.14$ & 0.64 \\
Tinetti Total & $26.5 \pm 1.4$ & $20.0 \pm 3.4$ & $<0.01^{*}$ \\
Tinetti Gait & $11.7 \pm 0.6$ & $9.0 \pm 1.7$ & $<0.01^{*}$ \\
Tinetti Balance & $14.9 \pm 1.2$ & $11.0 \pm 2.4$ & $<0.01^{*}$ \\
Fall history & $12 / 50$ & $24 / 50$ & $0.02^{*}$ \\
Walking speed (m/s) & $1.23 \pm 0.22$ & $0.86 \pm 0.26$ & $<0.01^{*}$ \\
Frequency (steps/s) & $1.86 \pm 0.17$ & $1.69 \pm 0.23$ & $<0.01^{*}$ \\
Step length (m) & $0.66 \pm 0.09$ & $0.51 \pm 0.13$ & $<0.01^{*}$ \\
Asymmetry (\%) & $6.05 \pm 5.55$ & $9.24 \pm 7.06$ & $0.02 *$ \\
Harmonic Ratio & $3.09 \pm 1.25$ & $2.18 \pm 1.09$ & $<0.01 *$ \\
Inter-stride Amplitude Variability & $0.08 \pm 0.03$ & $0.07 \pm 0.03$ & $<0.01 *$ \\
\hline$*$ Significant difference (p<0.05) between groups; Tinetti $\leq 24$ indicates at risk for falling, Tinetti $>24$ \\
indicates no fall risk & &
\end{tabular}

\section{Statistical Analysis}

ANOVA was used to examine differences in AGA-parameters between groups (Tinetti > 24 vs. 19-24 vs. < 19). Differences in fall history were studied by Fisher's exact test. Pearson correlation was performed to investigate associations between the Tinetti scale, AGA-parameters, fall history and subject demographics. Linear regression analysis was done to further explore associations between AGAparameters and the Tinetti scale, taking fall history and subject demographics into account. The discriminative power of AGA to differentiate subjects with a Tinetti $\leq$ 24 and $>24$ was assessed by determining the area under the Receiver Operating Characteristic (ROC) curve. The area under the curve (AUC), including 95\% confidence intervals, was used as a measure of the overall performance of a diagnostic test The closer AUC is to one, the better the overall diagnostic performance of the test (26). All analyses were done in SPSS 15.0. A p-value $\leq 0.05$ was considered significant.

\section{RESULTS}

The group with a Tinetti $\leq 24$ was significantly older and more often reported a fall in the six months prior to the experiment. The groups were similar in weight and height (table 1-2). The group with a Tinetti $\leq 24$ walked significantly slower, with shorter steps and lower step frequency than the group with a Tinetti $>24$. 
Moreover smaller RMS, larger step time asymmetry, lower harmonic ratio and smaller inter-stride amplitude variability was found in the group with a Tinetti $\leq 24$ (table 1). Comparable differences were found between risk groups with exception of the step frequency, step time asymmetry and inter-stride amplitude variability which were comparable between these groups (table 2).

All gait parameters correlated significantly with the Tinetti scale. The strongest correlation occurred for walking speed $(r=0.73, p<0.0)$ and the poorest for step time asymmetry $(r=-0.20, p=0.05)$. Walking speed, step length, RMS, step frequency, inter-stride amplitude variability and harmonic ratio correlated positively with the Tinetti scale (fig.2). A negative correlation was found with step time asymmetry (table 3 ). Age correlated moderately $(r=-0.59, p<0.01)$ and fall history weakly ( $r=-0.22, p=0.03$ ) with the Tinetti scale, while no correlation was found between the Tinetti scale and height $(p=0.17)$, weight $(p=0.27)$. Poor to excellent correlations were observed between AGA-parameters ( $r$-range: -0.20 (RMS vs. step time asymmetry) to 0.91 (RMS vs. walking speed), $p<0.05$ ).

AGA-parameters (except asymmetry and inter-stride amplitude variability) correlated with age, showing the lowest correlation for RMS $(r=0.38, p<0.01)$ and the highest correlation for walking speed $(r=0.55, p<0.01)$. Step time asymmetry was the only AGA-parameter correlating with fall history, showing a weak correlation ( $r=0.25, p=0.01$ ).

ROC analysis showed that walking speed, step length and RMS have excellent discriminative power to differentiate subjects with different Tinetti scores showing AUC ranging from 0.81 to 0.85 . Acceptable discriminative power was found for step frequency, harmonic ratio and inter-stride amplitude variability (AUC range: 0.71 0.73). Poor discriminative power ( $A \cup C=0.67$ ) was found for asymmetry.

Table 2: Subject demographics, Tinetti score, fall history and AGA-parameters for the groups with a Tinetti ranging between $19-24$ and the group with a Tinetti<24.

\begin{tabular}{lccc}
\hline & $\begin{array}{c}\text { Tinetti } \mathbf{1 9 - 2 4} \\
(\mathbf{n}=\mathbf{3 1})\end{array}$ & $\begin{array}{c}\text { Tinetti }<\mathbf{1 9} \\
(\mathbf{n}=\mathbf{1 9})\end{array}$ & p-values \\
\hline Age (yrs) & $76.0 \pm 4.8$ & $83.6 \pm 5.3$ & $<0.01^{*}$ \\
Height (m) & $1.69 \pm 0.09$ & $1.65 \pm 0.13$ & 0.19 \\
Weight (kg) & $72.9 \pm 14.1$ & $66.2 \pm 11.7$ & 0.08 \\
Tinetti & $21.9 \pm 1.7$ & $17.0 \pm 3.3$ & $<0.01^{*}$ \\
Fall History & $17 / 31$ & $7 / 19$ & 0.25 \\
Walking speed (m/s) & $0.95 \pm 0.24$ & $0.72 \pm 0.26$ & $<0.01^{*}$ \\
Frequency (steps/s) & $1.71 \pm 0.20$ & $1.64 \pm 0.28$ & 0.27 \\
Step length (m) & $0.55 \pm 0.11$ & $0.43 \pm 0.11$ & $0.01 *$ \\
Asymmetry (\%) & $9.39 \pm 8.14$ & $8.99 \pm 5.03$ & 0.84 \\
Root Mean Square & $0.18 \pm 0.07$ & $0.13 \pm 0.07$ & $0.03 *$ \\
Inter-stride Amplitude Variability & $0.07 \pm 0.03$ & $0.06 \pm 0.02$ & 0.21 \\
Harmonic Ratio & $2.51 \pm 1.13$ & $1.64 \pm 0.77$ & $0.01 *$ \\
\hline
\end{tabular}

*Significant difference $(p<0.05)$ between groups; Tinetti 19-24 indicates low fall risk, Tinetti < 19 indicates high fall risk. 

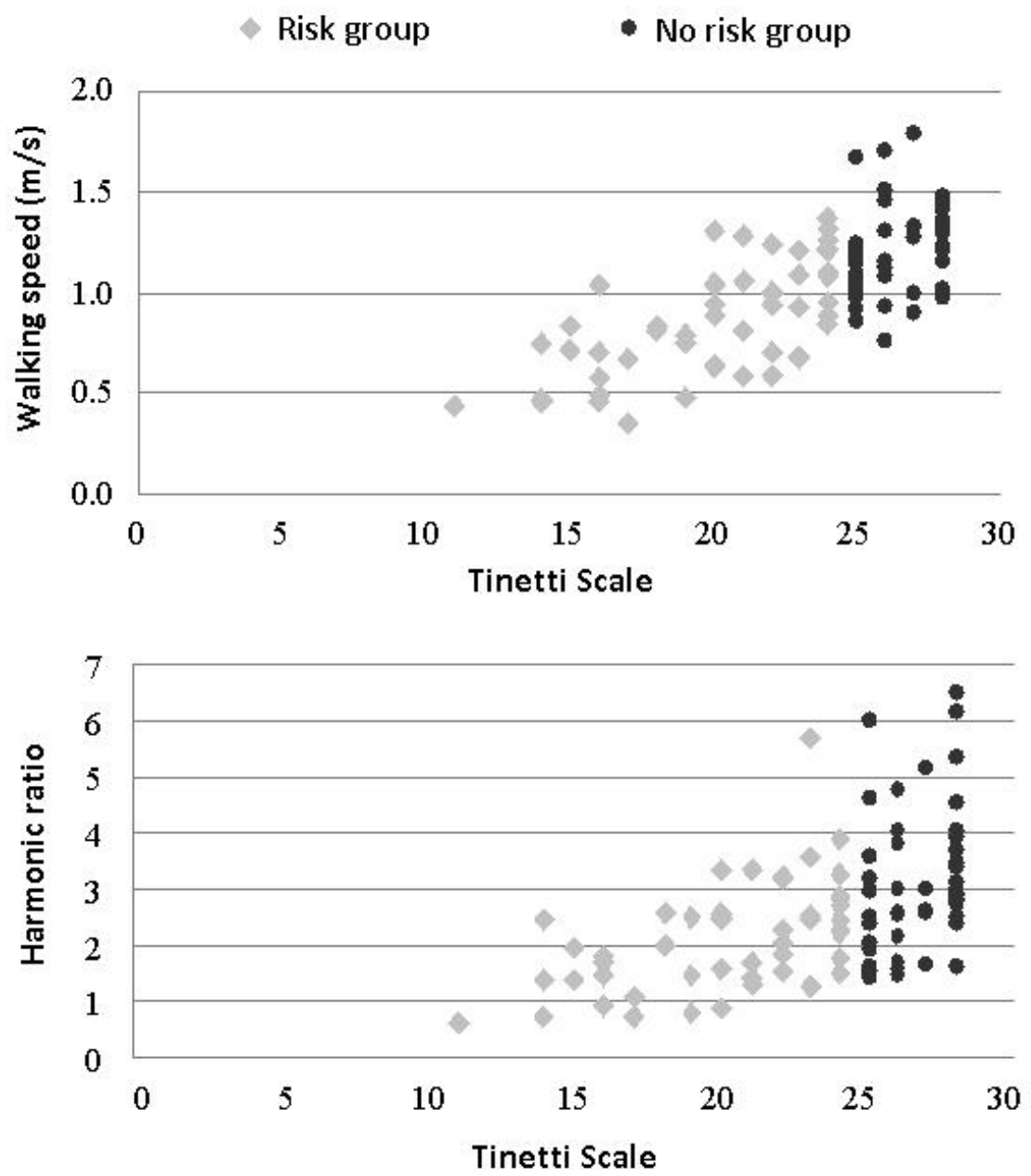

Fig. 2: Correlation plot Tinetti scale vs. walking speed (top) and harmonic ratio (bottom).

Table 3:Pearson correlation coefficients between AGA-parameters and the Tinetti scale and the Area Under the Curve (AUC) of the receiver operating characteristic analysis to determine the discriminative power of AGA to differentiate elderly with a Tinetti $\leq 24$ from the elderly with a Tinetti $>24$.

\begin{tabular}{lccc}
\hline & Correlation coefficient & AUC & 95\% Confidence Interval \\
\hline Walking speed (m/s) & $0.73(p<0.01)$ & 0.85 & $0.77-0.92$ \\
Frequency (steps/s) & $0.49(p<0.01)$ & 0.74 & $0.64-0.84$ \\
Step length (m) & $0.71(p<0.01)$ & 0.83 & $0.75-0.91$ \\
Asymmetry (\%) & $-0.20(p=0.01)$ & 0.67 & $0.63-0.83$ \\
Root Mean Square & $0.60(p<0.01)$ & 0.81 & $0.73-0.89$ \\
Harmonic Ratio & $0.52(p<0.01)$ & 0.73 & $0.64-0.83$ \\
Inter- stride Amplitude Variability & $0.33(p=0.01)$ & 0.71 & $0.61-0.81$ \\
\hline
\end{tabular}

\footnotetext{
* significant correlation $(p<0.05)$
} 


\section{Discussion}

This study investigated whether the Tinetti scale, as a commonly used subjective measure for fall risk, is associated with objectively measured gait characteristics. Clear differences in gait were found between the groups with different Tinetti scores. Walking speed, step length and RMS were the only AGA-parameters showing a strong association with the Tinetti scale. These parameters had also the best discriminative power to classify elderly according to the Tinetti scale.

The observed differences in AGA-parameters between groups were similar to previous studies that classified fall risk based on the PPA $(1,4)$ or Tinetti scale combined with fall history and functional measures (2). The slower walking speed and shorter steps in elderly with a Tinetti $\leq 24$ indicate that they adopt a more cautious gait pattern possibly to minimize upper body displacements (4, 27-28). Moreover this group showed impaired inter-stride amplitude variability, RMS and harmonic ratio. This implies for instance that elderly with a Tinetti $\leq 24$ have more difficulty in controlling gait smoothness and that they walk with more variability (4).

All AGA-parameters correlated with the Tinetti scale. However only walking speed and step length, which are highly correlated $(r>0.9, p<0.01)$, were both strongly associated with the Tinetti scale $(r>0.7, p<0.01)$. A faster walking speed was related to higher Tinetti scores, which corresponds to previous studies relating a slow walking speed with an increased fall risk $(2,4,29)$. Moreover walking speed and step length were the most powerful AGA-parameters to classify elderly according to the Tinetti scale (resp. AUC $85 \%$ and $83 \%$ ). The coordinates of the ROC curve of the walking speed showed that a cut-off level of $1.08 \mathrm{~m} / \mathrm{s}(3.89 \mathrm{~km} / \mathrm{h})$ is able to discriminate the group with a Tinetti $\leq 24$ from the group with a Tinetti $>24$ (76\% sensitivity, 70\% specificity). This cut-off value corresponds well with the study of Bautmans et. al. (cut-off $1.16 \mathrm{~m} / \mathrm{s}$, sensitivity $78 \%$, specificity $78 \%$ ), which also demonstrated that walking speed has the best discriminative capacity to classify elderly according to fall risk (2). RMS showed also good discriminative power to differentiate groups of elderly with different Tinetti scores (AUC 81\%). This parameter is highly correlated with the walking speed $(r=0.91, p<0.01)$ indicating redundancy (4). RMS is a useful proxy for the walking speed as it can be derived directly from the accelerometer data, not requiring additional measurements (e.g. distance) and calculations.

Many of the differences in AGA-parameters between groups appear to be related to differences in walking speed as is shown by their correlations ( $r$-range -0.25 $0.93, p<0.0)$. Consequently, harmonic ratio and inter-stride variability had no real additional value $(A \cup C<0.74, r<0.6, p<0.01$ ) to distinguish between the groups. These parameters may become more powerful and discriminative for other populations having more complex limitations like Parkinson Disease (30). This 
however needs further investigation and requires a reference database including norm values for AGA-parameters which may allow to identify fall-related gait characteristics in various population groups (22).

There was a small but significant difference in age between the risk groups and it could be argued that the gait differences are simply a consequence of age. It is known that gait changes with advancing age due to factors such as a loss in strength. However comparing AGA-parameters between the younger (65-74yrs) and older (75-84yr) subjects of each group, showed that the gait differences between the age groups were bigger in the group with a Tinetti $\leq 24$ than in the group with a Tinetti $>24$. This suggests that age is a factor, particularly in the group with a Tinetti $\leq 24$. However this needs further investigation including a larger amount of subjects per age (young vs. old) and fall risk group.

Linear regression analysis showed that only walking speed, step length and RMS are significant determinants of the Tinetti scale, with fall history and age only having small additional effects. Harmonic ratio and inter-stride amplitude variability had no additional value. The two best models, including speed $(B=7.76)$, age $(B=-0.19)$ and fall history $(B=-1.28)$ or RMS $(B=21.50)$, age $(B=-0.29)$ and fall history $(B=1.40)$ were able to explain respectively $60 \%$ and $52 \%$ of the variability in Tinetti scale. Analysis with fall history as an indicator for fall risk (logistic regression analysis) did not lead to better results $\left(R^{2}=0.09\right.$, with step time asymmetry as significant variable).

Interestingly, inter-stride amplitude variability and harmonic ratio which are frequently considered indicators for an elevated fall risk, had only moderate to poor associations with the Tinetti scale and were not incorporated in the regression models. Several aspects may explain the relatively poor association between the subjective and objective gait assessment. First of all, the population was categorized in two groups in advance (Tinetti $\leq$ and $>24$ ). In future studies, preferable prospective cross-sectional studies, a random inclusion of many elderly of varying subject characteristics and of varying Tinetti scores may provide further insight into these associations. However, in general the data was equally distributed over the studied ranges. Secondly, AGA-parameters were compared to the full Tinetti scale, which also includes a balance assessment (involves $57 \%$ of the Tinetti score). However, performing the analysis with the Tinetti-gait sub-score did not improve the observed associations (results not shown). It can be concluded that the small differences in gait that have previously been shown to be related to fall risk are not captured by the subjective assessment $(1,3-4,7)$. Hence, it may be worthwhile to include objective gait monitoring in fall risk screening (2).

This is one of the few studies that has included a large group $(n=100)$ of representative older subjects which had an average age of $77 \pm 6$ years (range 6590 years) that are categorized for fall risk using the Tinetti scale. However, a more comprehensive fall risk screening may be required to better understand the 
contribution of gait monitoring to fall risk. One of the issues in cross-sectional fall risk studies is that there isn't a real golden standard for fall risk. Fall risk is a multifactorial problem (11) and several aspects (e.g. medication use or fall history) were disregarded in this study. Interestingly, fall history, which is commonly used as an indicator of fall risk $(2,12)$ appeared to have only a small added values in this study. Screening various fall risk related aspects (e.g. Physiological Profile Assessment (11), fall history and gait assessment) in combination with a prospective evaluation of fall accidents (e.g. by fall detectors) (12) may strengthen a future study.

The findings of this study demonstrate the potential of AGA as objective gait analysis system in elderly at risk for falling. Detailed and objective information about changes and deviations in gait can be achieved by AGA. The practical properties of AGA (fast, easy, portable, approaching daily life conditions) will allow its use in clinical practice (e.g. nursing homes) in the future. Fall risk related AGAparameters may yield targets for fall prevention programs. Moreover AGA may help to make decisions regarding fall prevention and may allow to evaluate, optimize and develop fall prevention programs (e.g. Tai Chi). However currently there is no evidence that the Tinetti scale should be replaced by AGA, neither that fall risk assessment should rely on AGA. Future, preferably prospective studies are needed to indicate the most important and predictive variables for fall risk. The results of the current study will be the basis for these studies.

\section{Conclusion}

Small differences in gait that have previously been related to fall risk are not captured by the subjective fall risk assessment. It is therefore worthwhile to include objective gait assessment in fall risk screening. 


\section{RefERENCES}

1. Verghese J, Holtzer R, Lipton RB, Wang C. Quantitative gait markers and incident fall risk in older adults. J Gerontol A Biol Sci Med Sci 2009; 64A (8): 896-901.

2. Bautmans I, Jansen B, Keymolen van K, Mets T. Reliability and clinical correlates of 3Daccelerometry based gait analysis outcomes according to age and fall-risk. Gait Posture 2011; 33(3):366-372.

3. Lamoth CJ, Deudekom van FJ, Campen van JP, Appels BA, Vris de OJ, Pijnappels M. Gait stability and variability measures show effects of impaired cognition and dual tasking in frail people. J NeuroEngineering and Rehabilitation 2011; 8: 2-10.

4. Menz HB, Lord SR, Fitzpatrick RC. Acceleration patterns of the head and pelvis when walking are associated with risk of falling in community-dwelling older people. J Gerontol 2003; 58a: 446-52.

5. Fletcher PC, Hirdes JP. Risk factors for falling among community-based seniors using home care services. Journal of Gerontology Medical sciences 2002; 57A (8): M504-M10.

6. Ganz DA, Bao Y, Shekelle PG, Rubenstein LZ. Will my patient fall? Jama 2007; 297: 77-86.

7. Hausdorff JM, Edelberg HE, Mitchell SL, Goldberger AL, Wei JY. Increased gait unsteadiness in community dwelling elderly fallers. Arch Phys Med Rehabil 1997; 78: 278-83.

8. Brach JS, Berlin JE, VanSwearingen JM, Newman AB, Studenski SA. Too much or too little step width variability is associated with a fall history in older persons who walk at or near normal gait speed. Journal of NeuroEngineering and Rehabilitation 2005; 2: 20-8.

9. Tiedemann A, Shimada H, Sherrington C, Murray S, Lord S. The comparative ability of eight functional mobility tests for predicting falls in community-dwelling older people. Age Ageing 2008; 37: 430-4

10. Podsiadlo D, Richardson S. The timed up \& go: a test of basic functional mobility for frail elderly persons. J Am Geriatr Soc 1991; 39: 142-8.

11. Lord SR, Menz HB, Tiedemann A. A physiological profile approach to falls risk assessment and prevention. Physical Therapy 2003; 83 (3): 237-52.

12. Ganz DA, Higashi T, Rubenstein LZ. Monitoring falls in cohort studies of community-dwelling older people: effect of the recall interval. J Am Geriatr Soc 2005; 53: 2190-4.

13. Raîche M, Hebert R, Prince F, Corriveau H. Screening older adults at risk of falling with the Tinetti balance scale. Lancet 2000; 356: 1001-2.

14. Sterke CS, Huisman SL, Beeck van EF, Looman CWN, Cammen van der RJM. Is the Tinetti performance oriented mobility assessment (POMA) a feasible and valid predictor of short-term fall risk in nursing home residents with dementia? International Psychogeriatrics 2010; 22 (2): 254-63.

15. Lin MR, Hwang HF, Hu MH, Isaac HD, Wang YW, Huang FC. Psychometric comparisons of the timed up and go, one-leg stand, functional reach and Tinetti balance measures in community-dwelling older people. J Am Geriatr Soc 2004; 52: 1343-8.

16. Bloem BR, Hausdorff JM, Visser JE, Giladi N. Falls and freezing of gait in Parkinson's disease: a review of two interconnected, episodic phenomena Mov Disord 2004; 19: 871-84.

17. Kloos A, Kegelmeyer DA, Young GS, Kostyk SK. Fall risk assessment using the tinetti mobility test in individuals with Hungtington's disease. Mov Disord 2010; 25 (16): 2838-44.

18. Tinetti ME. Performance-oriented assessment of mobility problems in elderly patients. J Am Geriatr Soc 1986; 34: 119-26.

19. Senden R, Grimm B, Heyligers IC, Savelberg HHCM, Meijer K. An acceleration-based gait test for healthy subjects: Reliability and reference data. Gait Posture 2009; 30 (2): 192-6.

20. Senden R, Heyligers IC, Meijer K, Savelberg HHCM, Grimm B. Acceleration-Based Motion Analysis as a Tool for Rehabilitation: Exploration in Simulated Functional Knee Limited Walking Conditions. Am J Phys Med Rehab 2010; 90 (3): 226-32.

21. Hartmann A, Luzi S, Murer K, de Bie RA, de Bruin ED. Concurrent validity of a trunk tri-axial accelerometer system for gait analysis in older adults. Gait Posture 2009; 29: 444-8.

22. Senden R, Grimm B, Meijer K, Savelberg H, Heyligers IC. The importance to including objective functional outcomes in the clinical follow up of total knee arthroplasty patients. Knee 2011;18(5):306-311

23. Gietzelt M, Nemitz G, Wolf K, Schwabedissen H, Haux R, Marschollek M. A clinical study to assess fall risk using a single waist accelerometer. Informatics for Health \& Social Care 2009; 34 (4):181-8 
24. Zijlstra W, Hof AL. Assessment of spatio-temporal gait parameters from trunk accelerations during human walking. Gait Posture 2003; 18 (2): 1-10

25. Menz HB, Lord SR, Fitzpatrick RC. Acceleration patterns of the head and pelvis when walking on level and irregular surfaces. Gait Posture 2003; 18 (1): 35-46

26. Hosmer MJ, Bosscher RJ, Wieringen van PCW, eds. Applied Logistic Regression. Chichester: Wiley; 2000.

27. Nutt JG. Classification of gait and balance disorders. Adv Neurol 2001; 87: 135-141

28. Gehlesen GM, Whaley MH. Gait assessment in the elderly: a gait abnormality rating scale and its relation to falls. J Gerontol Med Sci 1990; 45: M12-M9

29. Kegelmeyer DA, Kloos AD, Thomas KR, Kostyk SK. Reliability and Validity of the Tinetti Mobility Test for Individuals with Parkinson Disease. Physical Therapy 2007; 87 (10): 1369-78

30. Latt MD, Menz HB, Funch VS, Lord SR. Acceleration patterns of the head and pelvis during gait in older people with Parkinson's disease: a comparison of fallers and nonfallers. J Gerontol. 2009;64A(6):700-6. 



\section{Chapter 7}

The 'TRiP', a specially designed experimental set-up to induce perturbations in a standardised way: A technical note

Senden R, Willems P, Savelberg HHCM, Grimm B, Heyligers IC, Meijer K. Submitted for publication 


\section{ABSTRACT}

To explore human stability during walking, an experimental set-up is required that can mimic the wide variety of perturbations that humans encounter during locomotion. To this aim a specially designed pneumatic braking device able to induce perturbations of varying duration, force and obstruction time, the Timed Rapid induced Perturbation (TRiP), was developed. This study investigated whether the TRiP meets its design requirements.

Five healthy young subjects walked on a treadmill while the TRiP set-up repeatedly perturbed the right leg. A standardized protocol was used to induce 16 perturbations in gait which varied in duration (range 50 - 200ms), force (braking pressure range 0.5 - 4 bar) and obstruction time (early and late in pre-swing phase). The protocol was performed at three different walking speeds. A force sensor attached at the ankle and in series with the breaking system and 6 Vicon MX3 cameras were used to evaluate the TRiP set-up. The response used to recover from the perturbations was visually assessed by video recordings.

The duration and obstruction time of the perturbation was reproduced within $15 \%$ accuracy from the set-value. Mainly perturbations with durations longer than $150 \mathrm{~ms}$ were reproduced with lower accuracy, showing an average underestimation of $72 \pm 22 \mathrm{~ms}$. A consistent time delay $(0.024 \mathrm{~s} \pm 0.008 \mathrm{~s})$ was observed between the synchronization pulse and the real occurrence of the perturbation. Perturbations of varying force could be induced by manually adjusting the pressure at the brake, showing higher perturbation forces with higher brake pressure $(R=0.87, p<0.05)$. A braking pressure lower than 1 bar did not induce a perturbation in gait. In addition, the TRiP elicited two stereotypical recovery strategies.

The TRiP is able to induce perturbations of varying characteristics in a controlled, semi-automatic way with moderate to good accuracy, in addition eliciting the two stereotypical recovery strategies. The TRiP set-up can thus be used to systematically explore walking stability in humans. 


\section{INTRODUCTION}

Perturbations in gait such as stumbles are one of the leading causes for fall incidents in the elderly population (1-2). However a perturbation in gait must not necessarily result in a fall as individuals have the capacity to compensate for the balance disturbance. The forward moment of the centre of mass created by the external perturbation can be stopped by a concerted action of the perturbed leg and the stance leg (3-7). The obstructed leg can be elevated in order to continue walking (elevating strategy) or the obstructed leg can be placed on the ground to take a recovery step with the contra-lateral leg (lowering strategy).

Studies exploring the limits of dynamic stability have probed the human muscular skeletal system with perturbations in controlled laboratory settings. Most studies are based on small population groups and a limited amount of perturbations induced by different approaches. Perturbations have been evoked by inducing an impact at the level of the foot (8-9), at the level of the ankle (10-11) or at the level of the waist (12). For instance, perturbations can be applied by obstacles released on the treadmill $(8-9,13-14)$ or lifted up from the floor while free walking (13-14), by blocking the swinging leg using tripping leashes while treadmill walking (10) or free walking on a walkway (11) or by reversal of the treadmill speed (15). These studies have shown that the recovery response is partly dependent on the characteristics of the perturbation, and more specific on the duration and obstruction time of the perturbation. For instance, perturbations induced early (late) in swing are mainly recovery by an elevating (lowering) strategy. While the obstruction time $(8,10,13)$ and the duration of the perturbation (10) could be controlled by these set-ups, they were unable to control the force of the perturbation. This may however be important to evoke perturbations that are representative for daily life situations, for instance to simulate the difference between stumbling upon a concrete side walk vs. a compliant carpet. To investigate the limits of dynamic stability under controlled conditions in bigger population groups including perturbations in gait of varying force, a specially designed laboratory-based pneumatic braking device (IDEE), the Timed Rapid induced Perturbations (TRiP), was developed. The TRiP set-up includes a treadmill instrumented with two specially designed breaking systems (TRiP-devices), which enables to induce a wide range of different perturbations.

The current study investigates whether the TRiP set-up meets the design requirements which are 1 ) to induce perturbations in a controlled way, 2) to induce perturbations with varying duration, 3 ) to induce perturbations at varying times in the gait cycle, 4) to induce perturbations of varying force and 5) to elicit two stereotypical recovery strategies (elevating and lowering strategy). 


\section{Methods}

Five healthy young subjects (1F/4M, ranges: 23 - 33 yrs, $59-85 \mathrm{~kg}, 1.67-1.94 \mathrm{~m}$ ) walked on a treadmill, while the TRiP-device was attached to the right leg of the subject. All volunteers were healthy, ensuring that the gait and recovery performance was not influenced by health limitations. The study was approved by the local Medical Committee and all subjects gave informed consent.

The TRiP set-up comprises 1) a treadmill (Medifit) equipped with a safety harness and an emergency stop and 2) the TRiP, a specially designed pneumatic braking device (IDEE) to induce controlled trips (Fig. 1). One TRiP-device consists of an axle attached to a PC controlled pneumatic brake and an electric motor with encoder. A flexible rope with low strain and high yield strength (Dynema ${ }^{\circledR}$ ) is attached to the ankle of the subject and the axel of the TRiP. The rope winds and unwinds during each stride. The motor keeps the ropes taught at a force of approximately $2 \mathrm{~N}$, while the encoder reads out the displacement of the ankle. The microcontroller continuously monitors the gait cycle via the different signals between the encoders and uses this information to trigger a perturbation at specific, predefined phases of the swing phase by briefly blocking the legs using the rope $(0 \%$ toe off, $100 \% \mathrm{mid}$ swing, $0 \%$ heel strike). Specially designed software controls the duration of the perturbation, which can vary between 50 - $250 \mathrm{~ms}$ and the timing of the obstruction. Perturbations can only be induced during the first half of the swing phase, because no reaction is elicited for perturbations in the second half of the swing phase which is due to the continuously moving of the stance leg because of the treadmill. The braking force can be adjusted manually via a pressure valve (0 - 5 bar). Finally the specially designed software also sends a synchronisation pulse to a camera, that triggers the camera to record.

The experiment consisted of two parts and took more or less 40 minutes per subject. In the first part, which was completely automatic, the duration of the perturbation and the timing of the obstruction was adjusted while the braking pressure was fixed at 3 bar. Four perturbations were induced with a fixed duration of $150 \mathrm{~ms}$, while the timing of the obstruction varied from $20 \%$ to $80 \%$ of the first half of the swing phase, with a $20 \%$ interval. Perturbations induced at $20 \%$ (80\%) of the first half of the swing phase are defined as early (late) swing perturbations (Fig. 2). Another four perturbations were induced at $50 \%$ of the first half of the swing phase, while the duration varied $(50 \mathrm{~ms}, 100 \mathrm{~ms}, 150 \mathrm{~ms}$ or $200 \mathrm{~ms})$. In the second part, the braking pressure was manually adjusted. Eight perturbations of different braking pressure, varying from 0.5 bar to 4 bar with an interval of 0.5 bar, with a fixed duration of $150 \mathrm{~ms}$ and induced at $50 \%$ of the first half of the swing phase were elicited per subject. The sequence at which the perturbations were evoked was randomly chosen, but was identical for all subjects. This standardized protocol of 16 perturbations was performed at three different walking speeds starting with 

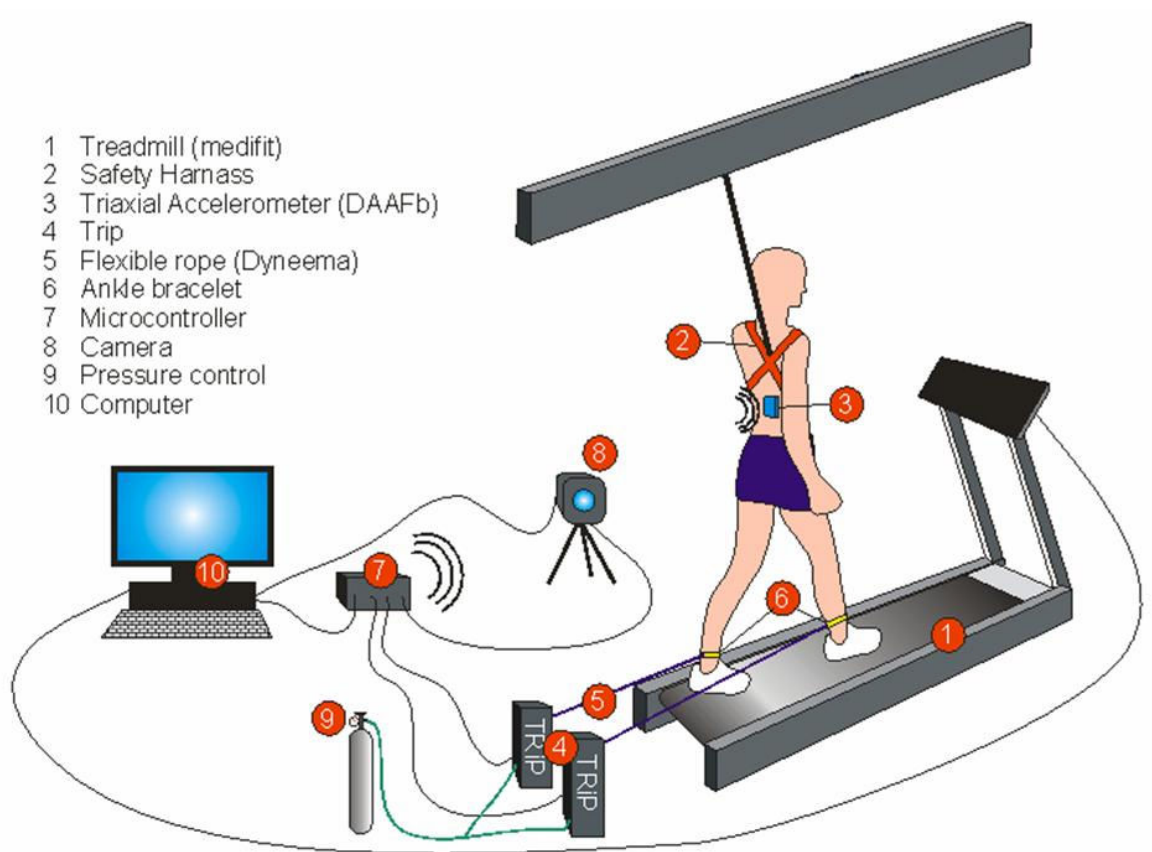

Fig. 1: The Timed Rapid Impact Perturbation (TRiP) set-up, used to evoke perturbations in a standardized and controlled way.

The triaxial accelerometer shown in this figure is not part of the TRiP set-up. The accelerometer, as presented here, was used to investigate the influence of the trip on gait, which showed to have no effect

$3 \mathrm{~km} / \mathrm{h}$, increasing to $4.5 \mathrm{~km} / \mathrm{h}$ and ending with $6 \mathrm{~km} / \mathrm{h}$. This resulted in a total 240 perturbations (48 perturbations per subject).

The time between successive perturbations was sufficient (minimal 20s) to enable subjects to recover completely and to regain their normal walking pattern. To become accustomed to the TRiP set-up, subjects walked for two minutes undisturbed on the treadmill while being attached to the TRiP-device before the experiment started. In a pilot study on 10 healthy subjects (5 young and 5 older subjects), treadmill walking at preferred speed (avg. $1.18 \pm 0.25 \mathrm{~m} / \mathrm{s}$ ) with and without the TRiP device was compared using acceleration-based gait analysis (not published data). No significant differences in gait parameters were found between both walking conditions (e.g. step time of $0.53 \pm 0.05 \mathrm{~s}$ vs. $0.52 \pm 0.05 \mathrm{~s}$, step length $0.62 \pm 0.14 \mathrm{~m}$ vs. $0.61 \pm 0,14 \mathrm{~m}$, walk ratio $0.56 \pm 0.14 \mathrm{~m} / \mathrm{Hz}$ vs. $0.54 \pm 0.14 \mathrm{~m} / \mathrm{Hz}$ for respectively treadmill walking without and with the TRiP device).

A force sensor (smart junior S-beam 500N, $17 \times 7 \times 19 \mathrm{~mm}, 12 \mathrm{~g}$ ) attached at the ankle and in series with the rope was used to measure the perturbation force (N) and to determine the duration of the perturbation. Six Vicon MX3 camera's $(100 \mathrm{~Hz})$ 

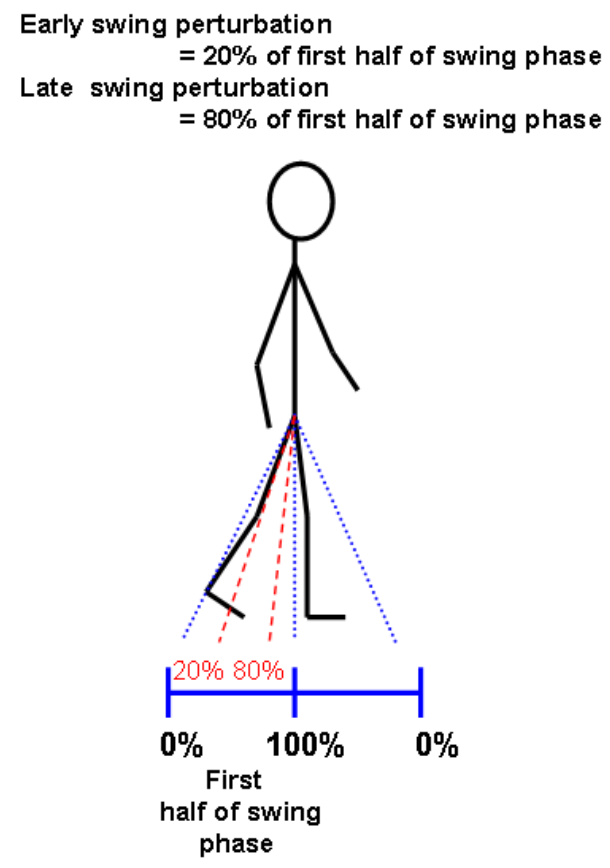

Fig. 2: Perturbations applied during the first half of the swing phase.

The dotted blue lines represent the swing phase of the gait cycle which starts with toe off $(0 \%)$, followed by mid swing (100\%) and ending with heel strike (0\%). Perturbations were induced during the first half of the swing phase (toe off - mid swing\%); at $20 \%$ and $80 \%$, as is shown by the red dotted lines

were used to capture 3D motion and was conducted during a four second time window around the perturbation. Prior to the experiment, markers were placed at Bony landmarks according to the specifications of the VICON lower limb model. This data was used to determine the timing of the obstruction during swing. The whole experiment was recorded on video (2D) which served to retrospectively assess the recovery strategy. A pilot study in which the recovery strategy of 300 perturbations was assessed by video recordings, showed that this method of assessing had an overall agreement of $\pm 93 \%$ between three trained observers ( \pm 7\% deviation).

\section{Statistical anAlysis}

To test the design requirements, the difference between the set-values and the actual features of the perturbation was determined. Regression analysis was used to investigate the relation between the braking pressure and the perturbation 
force. ANOVA was used to examine the effect of walking speed on the accuracy of the TRiP. Analysis was done in SPSS Version 15.0, using 0.05 as significance level.

\section{RESULTS}

In total 240 perturbations were induced of which 11 perturbations were excluded due to practical issues (e.g. release of the tripping leashes), leaving a total of 229 perturbations for analysis. In addition, due to technical limitations of the force sensor, the analysis concerning the induced force at the ankle is based on 3 subjects (139 perturbations).

Perturbation forces ranged from $54 \mathrm{~N}$ to $313 \mathrm{~N}$. No change in force at the ankle with regard to unperturbed walking $(2 \mathrm{~N})$ was observed for perturbations with a set braking pressure of 1.0 bar and lower. The average perturbation force was $194 \mathrm{~N} \pm$ $48 \mathrm{~N}$. A strong significant correlation was found between the braking pressure and the perturbation force showing increasing perturbation force with increasing braking pressure $\left(R^{2}=0.74, p<0.01\right.$, Fig. 3$)$. ANOVA found a modulating effect of the walking speed on the perturbation force, showing increasing perturbation forces at higher walking speeds $(176.4 \mathrm{~N}, 196.9 \mathrm{~N}$ and $207.2 \mathrm{~N}$ for respectively $3,4.5$ and $6 \mathrm{~km} / \mathrm{h}, \mathrm{p}=0.02$ ). Post hoc Bonferroni analysis showed that the perturbation force was significantly higher at $6 \mathrm{~km} / \mathrm{h}$ compared to $3 \mathrm{~km} / \mathrm{h}$.

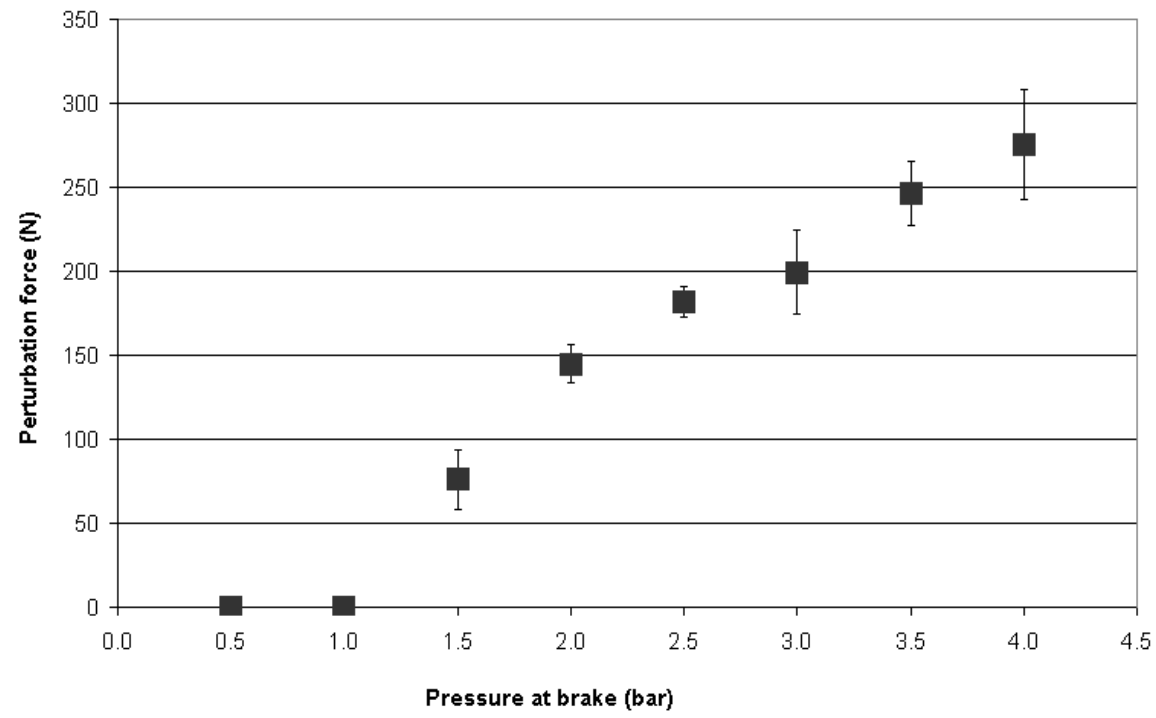

Fig. 3: The brake pressure correlated positively with the perturbation force $(p<0.05)$. 


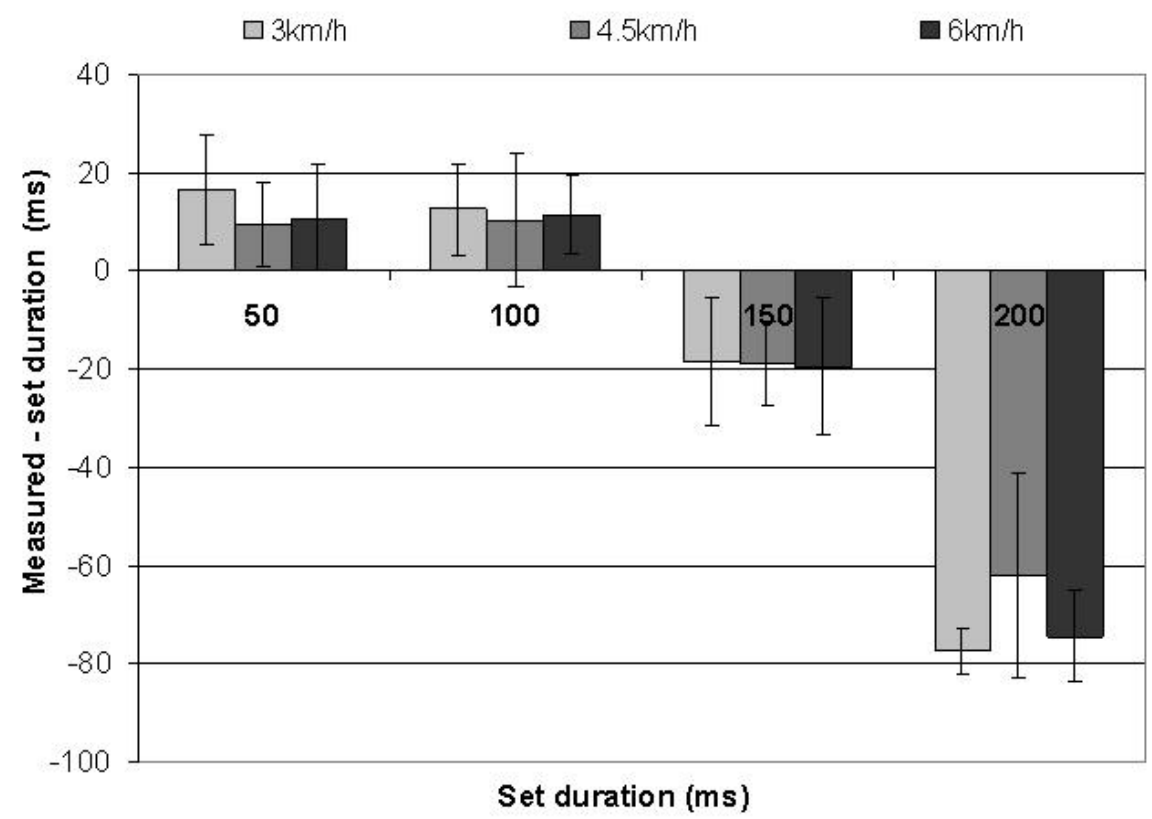

Fig. 4: Difference in perturbation duration between set-value and actual perturbation for varying set durations.

The duration of the perturbation was reproduced within $15 \%$ accuracy from the set-value (Fig. 4). Perturbations with a duration of $100 \mathrm{~ms}$ and shorter showed a slight overestimation compared to the set-values of $13 \mathrm{~ms} \pm 7 \mathrm{~ms}$. Perturbations with a set duration of $150 \mathrm{~ms}$ deviated on average $26 \mathrm{~ms} \pm 17 \mathrm{~ms}$ from the setvalues, which could be both over- and underestimated. Lower accuracy was found for perturbations with a duration of $200 \mathrm{~ms}$. On average they lasted $72 \mathrm{~ms} \pm 22 \mathrm{~ms}$ less than the set duration (deviation of $35 \%$ from set-value). No effect of walking speed on the deviation in perturbation duration was found (relative difference with set-values of $13.6 \%, 14.7 \%$ and $15.4 \%$ for respectively $3,4.5$ and $6 \mathrm{~km} / \mathrm{h}, \mathrm{p}=0.706$ ). The timing of the obstruction differed on average 15\% $\pm 7 \%$ (range $0-33 \%$ ) from the set-value. No modulating effect of the walking speed was found (deviation in obstruction time of $17 \%, 14 \%, 15 \%$ for respectively $3,4.5$ and $6 \mathrm{~km} / \mathrm{h}, \mathrm{p}=0.129$ ). However a time delay observed between the synchronization pulse and the real occurance of the perturbation contributes to this deviation. This delay was consistent $(0.024 \mathrm{~s} \pm 0.008 \mathrm{~s})$ across walking speeds and for perturbations of varying duration and obstruction time (e.g. deviation of $12 \pm 9 \%, 16 \pm 7 \%, 15 \pm 5 \%$ and $16 \pm$ $7 \%$ for respectively perturbations induced at $20 \%, 40 \%, 60 \%$ and $80 \%$ of the first half of the swing phase; Fig. 5).

The Trip elicited both the lowering and the elevating recovery strategy. However only a clear recovery strategy was evoked if the induced perturbation force was 
bigger than $105 \mathrm{~N}$ (corresponding to braking pressure $>1.5 \mathrm{bar}$ ) and the perturbation duration was longer than $50 \mathrm{~ms}$ (corresponding perturbation force $138 \mathrm{~N}-192 \mathrm{~N}$ ). Of the remaining 180 perturbations, $44 \%$ of the perturbations were recovered by an elevating strategy. Additional analysis including only perturbations with a fixed duration of $150 \mathrm{~ms}$ and pressure brake of 3 bar, but with varying obstruction times ( $n=60$ perturbations) showed that $67 \%$ of the early swing perturbations were recovered by an elevating strategy and that $93 \%$ of the late
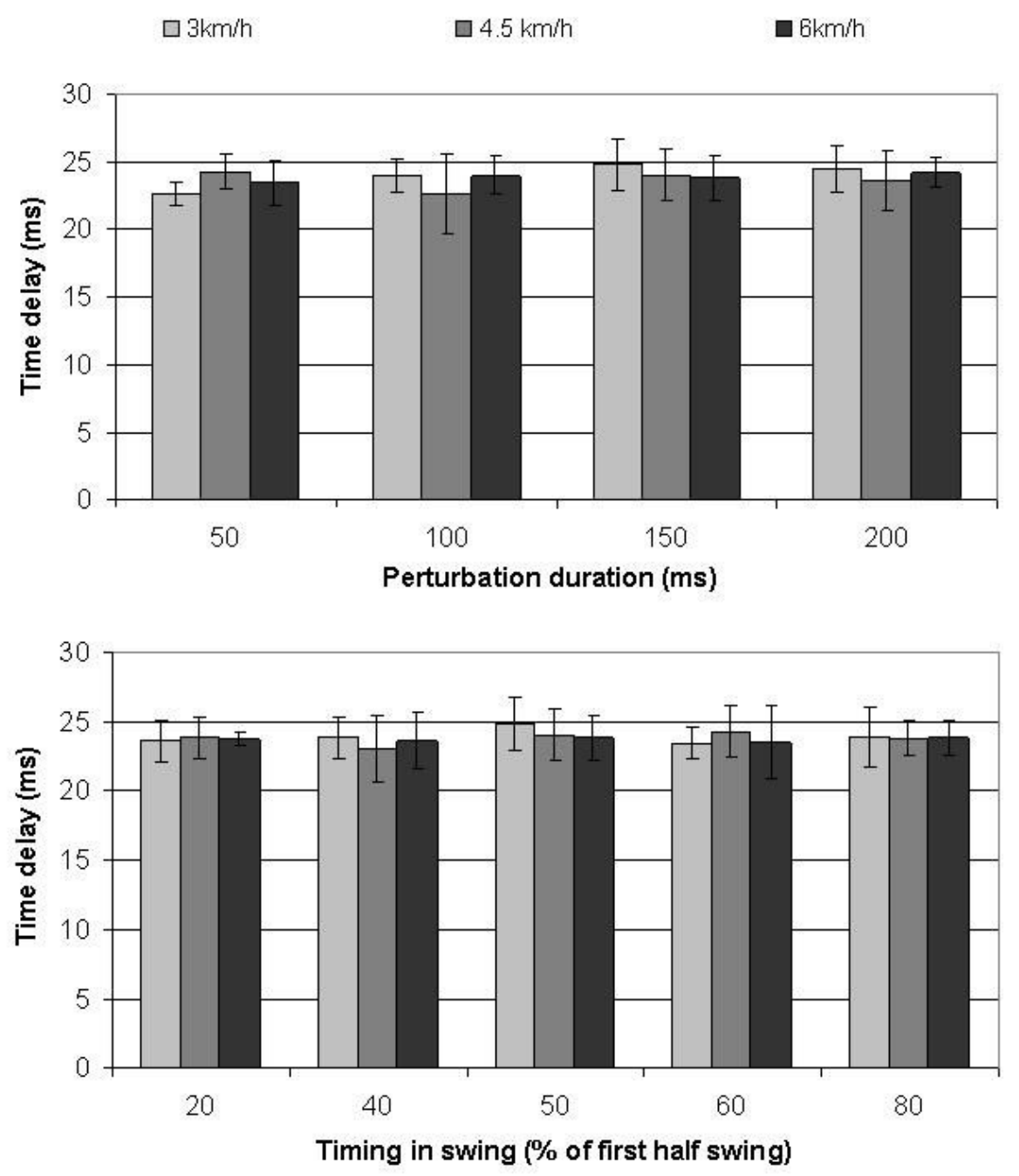

Fig. 5: The time delay measured for various perturbations of different perturbation durations (top; fixed brake pressure of 3 bar and obstruction time of $50 \%$ of first half of swing phase) and of different obstruction times (bottom; fixed duration of $150 \mathrm{~ms}$ and brake pressure of 3 bar). 
swing perturbations were recovered by a lowering strategy. This indicates an effect of obstruction time on the recovery strategy. Also an effect of walking speed was observed, showing significantly fewer perturbations recovered by an elevating strategy when walking at $6 \mathrm{~km} / \mathrm{h}$ (20\% of the perturbations) compared to $3 \mathrm{~km} / \mathrm{h}$ (77\% of the perturbations). The recovery strategy was not affected by the force and duration of the perturbation.

\section{Discussion}

This study investigated whether the specially designed TRiP set-up meets the design requirements. The TRiP set-up is able to reproduce perturbations with specific features in terms of duration, obstruction time and force in a controlled, semi-automatic way with moderate to good accuracy. In addition both stereotypical recovery strategies could be elicited by the TRiP.

The accuracy in obstruction timing can be improved by correcting for the consistent time delay (avg. $0.024 \mathrm{~s} \pm 0.008 \mathrm{~s}$ ) between the synchronization pulse and the real occurrence of the perturbation. Such a correction results in a $5.6 \%$ improvement, indicating that the TRiP set-up is able to induce perturbations at various obstruction times within $10 \%$ accuracy from the set-value. This deviation is acceptable in order to induce perturbations in early and late swing.

The deviation in perturbation duration was mainly attributed to the low accuracy during the $200 \mathrm{~ms}$ blocking conditions. Reflex mediated factors may play a role (15) as the recovery process (e.g. placement of obstructed leg on ground) may have started before the 200ms perturbation duration was elapsed, resulting in shorter perturbation durations than the set $200 \mathrm{~ms}$. Additional analysis excluding perturbations with a duration of $200 \mathrm{~ms}$ showed that the accuracy in perturbation duration improved to $97 \%$. On average, perturbation duration was now slightly overestimated (avg. $3 \mathrm{~ms} \pm 1 \mathrm{~ms}$ ).

In contrast to other perturbation set-ups, the TRiP allows for control of the perturbation force $(8-9,10,13)$. This enables the TRiP to simulate perturbations of different compliance which can be done by manually adjusting the braking pressure. For instance, a serious perturbation in gait, (e.g. tripping over a pavement while walking fast) can be simulated by setting a high pressure at the brake, while a small disturbance (e.g. shoe that hold back on the ground while strolling) can be simulated by setting a low pressure at the brake. The observed positive relation between perturbation forces and walking speed can be associated with the higher accelerations of the swinging leg when walking at increasing speed.

Both stereotype recovery strategies were elicited by the TRiP set-up. Corresponding to previous studies, the choice for one of the two strategies depended partly on the obstruction time that the perturbation occurred in the 
swing phase. Previous studies have shown that lowering strategies occur mainly during late swing perturbations (5). Also the walking speed had an influence on the recovery strategy, showing fewer elevating strategies at faster walking speeds. The higher walking speed causes the swinging leg to move with higher accelerations which increases the impact of the brake resulting in a direct placement of the leg on the ground. In addition, perturbation forces of $105 \mathrm{~N}$ did not induce a clear recovery strategy, although a deviation in the gait pattern was visually observed (e.g. hesitation in swing leg). Also perturbations of $50 \mathrm{~ms}$ did not induce a clear recovery response while they did induce an impact at the ankle that should evoke a clear recovery strategy (force range $138 \mathrm{~N}-192 \mathrm{~N}$ ). Based on this data it can be concluded that a perturbation of at least $50 \mathrm{~ms}$ with a blocking force larger than $105 \mathrm{~N}$ is required to evoke one of the two stereotypical recovery strategies. No visual change in the gait pattern with respect to unperturbed treadmill walking was observed if perturbations with a braking pressure of 1 bar and lower were induced. These perturbations did not put any additional force at the ankle (force of $2 \mathrm{~N}$, similar to unperturbed treadmill walking with TRiP device), indicating that a braking pressure bigger than 1 bar is required to induce a perturbation in gait. No real upper limit was found at which the perturbation characteristics did not have any influence anymore on the perturbation force. The perturbation force increased between every braking pressure interval (from 1 bar till 4 bar with 0.5 bar interval) and levelled off at a braking pressure of 3.5 bar and 4 bar. It can therefore be suggested that the perturbation force further increases for perturbations with braking pressures higher than 4 bar. Also the upper limit of the obstruction timing could not be investigated because perturbations were only induced in the first half of the swing phase. All results are based on data of 5 healthy young subjects. More research is needed including a large group of young and older subjects, to investigate the lower and upper limits of perturbations and to examine how the recovery response to perturbations in gait are influenced by perturbation characteristics (e.g. perturbation duration and force), subject characteristics (e.g. age) and other factors like physiological factors (e.g. muscle strength).

While the TRiP set-up is able to induce perturbations of varying characteristics, the TRiP set-up has also some limitations. Because the tripping leashes become loose late in the swing phase, the TRiP allows only to induce perturbations in the first half of the swing phase. This limits to explore perturbations provoked in the second half of the swing phase. Secondly, a resistance was continuously present on the tripping leashes to keep them thigh which is required in order to avoid stepping on the leashes and to induce the perturbation on time. This resistance made walking more demanding than normal. However, in a pilot study it was shown that this resistance had no significant effect on the walking pattern of the subjects. Finally, the treadmill may be a limiting factor because it obliged subjects to keep walking at a certain walking speed, even during the recovery process. The treadmill also 
provides that the perturbed and the standing leg move respectively forward and backwards with regards to the treadmill, which may influence the responses to perturbations. However because both recovery strategies could be evoked, the disadvantage of the treadmill seems negligible. Even more, the use of a treadmill was beneficial because it allowed to induce perturbations at predefined phases in the gait cycle, which is partly due to the stable step length. The TRiP set-up can further be optimised by automatically compensating for the time delay found between the synchronization pulse and sthe real occurrence of the perturbation and by further investigating relationships between perturbation force, duration, obstruction time and walking speed.

\section{CONCLUSION}

The TRiP, a specially designed pneumatic braking device, is able to induce perturbations of varying characteristics in a controlled, semi-automatic way with moderate to good accuracy, in addition eliciting the two stereotypical recovery strategies. The TRiP set-up can therefore be used to systematically explore walking stability in humans. 


\section{REFERENCES}

1. Srygley JM, Herman T, Giladi N, Hausdorff JM. Self-report of missteps in older adults: A valid proxy of fall risk? Arch Phys Med Rehabil 2009 692;90:786.

2. Berg WP, Alessio HM, Mills EM, Tong C. Circumstances and consequences of falls in independent community-dwelling older adults. Age Ageing 1997;26(4):261-8.

3. Pijnappels $M$, Bobbert MF, van Dieën JH. Push-off reactions in recovery after tripping discriminate young subjects, older non-fallers and older fallers. Gait Posture 2005;21(4):388-94.

4. Grabiner MD, Koh TJ, Lundin TM, Jahnigen DW. Kinematics of recovery from a stumble. J Gerontol A Biol Sci Med Sci 1993;48:M97-102.

5. Eng JJ, Winter DA, Patla AE. Strategies for recovery from a trip in early and late swing during human walking. Exp Brain Res 1994;102:339-49.

6. Forner-Cordero A, Koopman HJFM, van der Helm FCT. Multiple-step strategies to recover from stumbling perturbations Gait Posture 2004;18:47-59.

7. Schillings $A M$, Wezel van $B M H$, Mulder T, J D. Muscular responses and movement strategies during stumbling over obstacles. J Neurophysiol 2000;83:2093-102.

8. Weerdersteyn V, Schillings AM, van Galen GP, Duysens J. Distraction affects the performance of obstacle avoidance during walking. J Mot Behav 2003;35:53-63.

9. Schillings AM, Mulder T, Duysens J. Stumbling over obstacles in older adults compared to young adults. J Neurophysiol 2005;94:1158-68.

10. Forner-Cordero A, Koopman HJFM, Helm van der FCT. Multiple-step strategies to recover from stumbling perturbations. Gait Posture 2004;18:47-59.

11. Smeesters C, Hayes WC, McMahon TA. The threshold trip duration for which recovery is no longer possible is associated with strength and reaction time. J Biomech 2001;34:589-95.

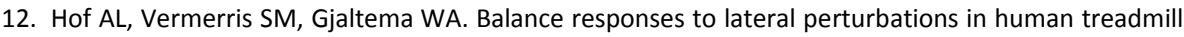
walking. J Exp Biolog 2010;213:2655-64.

13. Pijnappels $M$. Push-off reactions in recovery after tripping discriminate young subjects, older nonfallers and older fallers. Gait Posture 2005;21(4):388-94.

14. Pavol MJ, Owings TM, Foley KT, Grabiner MD. Influence of lower extremity strength of healthy older adults on the outcome of an induced trip. JAGS 2002;50(2):256-62.

15. Granacher U, Gruber M, Dominik F, Strass D, Gollhofer A. Effects of ankle fatigue on functional reflex activity during gait perturbations in young and elderly men. Gait Posture 2010;32:107-12. 



\section{Chapter 8}

\section{The influence of age, muscle strength and speed of information processing on recovery responses to external perturbations in gait}

Senden R, Savelberg HHCM, Adam JJ, Grimm B, Heyligers IC, Meijer K. Submitted for publication 


\section{ABSTRACT}

Dynamic imbalances caused by external perturbations in gait can successfully be counteracted by adequate recovery responses. The current study investigated how the recovery response is moderated by age, walking speed, muscle strength and speed of information processing. Gait of 50 young and 45 elderly subjects was repeatedly perturbed at $20 \%$ and $80 \%$ of the first half of the swing phase using the Timed Rapid impact Perturbation (TRiP) set-up. Recovery responses were identified using 2D camera. Muscular factors were measured using a dynamometer and speed of information processing parameters were determined using a computerbased four-choice finger-cuing reaction time task. The stronger, fast reacting and fast walking young subjects recovered more often by elevating strategy than elderly subjects. Differences in recovery responses were explained for $23 \%$ by a combination of walking speed $(B=-13.85)$, speed of information processing $(B=-$ $0.82)$, maximum extension strength $(B=0.01)$ and rate of extension moment development $(B=0.19)$. The recovery response that subjects employed when gait is perturbed by the TRiP set-up was modified by several factors, in which the individual contribution of walking speed, muscle strength and speed of information processing was small. Insight into remaining modifying factors is needed to assist and optimise fall prevention programs. 


\section{INTRODUCTION}

The rapidly growing elderly population entails an increasing fall incidence which has serious consequences for both the individual and the health care systems (1). Near falls, like trips, are indicated as relevant markers of fall risk (2); they account for approximately $59 \%$ of the falls in elderly (3). A near fall is defined as a temporary disturbance in dynamic balance which is caused by external perturbations. Healthy individuals have the remarkable capacity to counter the forward momentum of the centre of mass which results from an external perturbation, by a concerted action of the perturbed leg and supporting leg (4-5). In general two recovery strategies have been identified, either the obstructed leg is placed on the ground immediately after being perturbed while a recovery step is taken with the contra-lateral leg (lowering strategy, LS), or the obstructed leg can be elevated after being perturbed in order to continue walking (elevating strategy, ES).

Previous studies have shown that the choice for the recovery response depends on the perturbation characteristics, e.g. perturbation duration (6) and obstruction timing in the gait cycle (4-9). For instance early swing perturbations mainly evoke ES, while late swing perturbations mainly evoke LS. Interestingly, similar perturbation conditions yield different recovery responses in young and elderly subjects (8-9). Studies have shown that elderly recover more often by LS (8-9), which has been attributed to e.g. an impaired limb positioning and reduced lower limb strength (9). Moreover studies found that elderly are less successful in their recovery as is indicated by higher failure rates, which is defined as taking additional steps, having secondary contacts with the obstacle and even fall events. For instance the study of Pijnappels et. al. showed that young subjects recovered successfully from mid swing perturbations by ES, while elderly used ES and LS to recover from identical perturbations, but failed more often in their recovery (8). The less adequate recovery responses in elderly have been associated with age related physiological changes such as lower peak moments, poorer placement of the recovery limb, reduced response time, etc. $(4,6,8-12)$. Most studies on balance recovery focus on success/failure rates, are conducted in small groups and/or rely on limited amount of perturbations and therefore investigating mainly a single physiological aspect at a time. For instance, Schillings et. al. investigated muscular responses during stumbling over obstacles (4) and Lamoureaux et. al. examined the effect of muscle strength on obstacle negotiation (10). In daily life, elderly have to deal simultaneously with several age related physiological changes such as an impaired speed of information processing and reduced muscle strength. Currently it is unclear how these changes and the combined effect of these changes are associated with recovery responses. It is hypothesized that there is a graded response to external perturbations, where physiological deterioration is initially 
coped with by relying on the LS and changes in gait. In later stages failure to recover is expected to become more prominent. The current study aims to investigate how the recovery response to external perturbations in gait is moderated by age, walking speed, muscle strength and speed of information processing, relying on a sizable population of young and elderly subjects and a large amount of controlled perturbations.

\section{Materials ANd Methods}

\section{Subjects}

Fifty healthy young $(23 \mathrm{M} / 27 \mathrm{~F}, 24 \pm 4 \mathrm{yrs})$ and 45 healthy elderly $(20 \mathrm{M} / 25 \mathrm{~F}, 67 \pm 6 \mathrm{yrs}$, table 1) subjects were included. Exclusion criteria were cardiac problems, breathing problems, diabetes, neurological diseases, hearing or sight impairments, use of psychoactive or sedative medication, use of walking aid, unable to walk or Tinetti score $<24$ indicating at risk for falling. The study was approved by the local ethical committee; all participants were informed and signed informed consent before participating.

\section{Measurements and outcomes}

\section{1) TRiP experiment}

The 'Timed Rapid Impact Perturbation' (TRiP) set-up, a specially designed trip setup consisting of a treadmill (Medifit) equipped with a safety harness and two pneumatic braking devices was used to induce perturbations in a standardized way (Fig 1.) (13). The TRiP can perturb the swinging leg at specific instants during the first half of the swing phase, with specific blocking durations and perturbation forces, thereby triggering LS and ES (online supplement, (13)). A fixed protocol was used to induce 10 perturbations at $20 \%$ (early swing perturbations) and 10 perturbations at $80 \%$ (mid swing perturbations) of the first half of the swing phase (Fig 2). Perturbations were equally distributed over both legs, had all a fixed duration of $150 \mathrm{~ms}$ and were induced with a fixed braking pressure of 3 bar. The time between successive perturbations was sufficient to enable subjects to regain their normal walking pattern. Video recordings were used to assess the recovery strategy. A pilot study on 300 perturbations showed that this assessment method has an overall agreement of $93 \%$ between instructed observers. The proportion perturbations recovered by an ES (\%) assessed over the complete experiment was used as a measure for the recovery response.

Prior to the TRiP experiment, subjects walked a $20 \mathrm{~m}$ straight distance to determine the comfortable walking speed. This speed was used during the TRiP experiment as a faster or slower speed may have an effect on the recovery response (13). 
1 Treadmill (medifit)

2 Safety Harnass

3 Triaxial Accelerometer (DAAFb)

4 Trip

5 Flexible rope (Dyneema)

6 Anke bracelet

7 Microcontroller

8 Camera

9 Pressure control

10 Computer

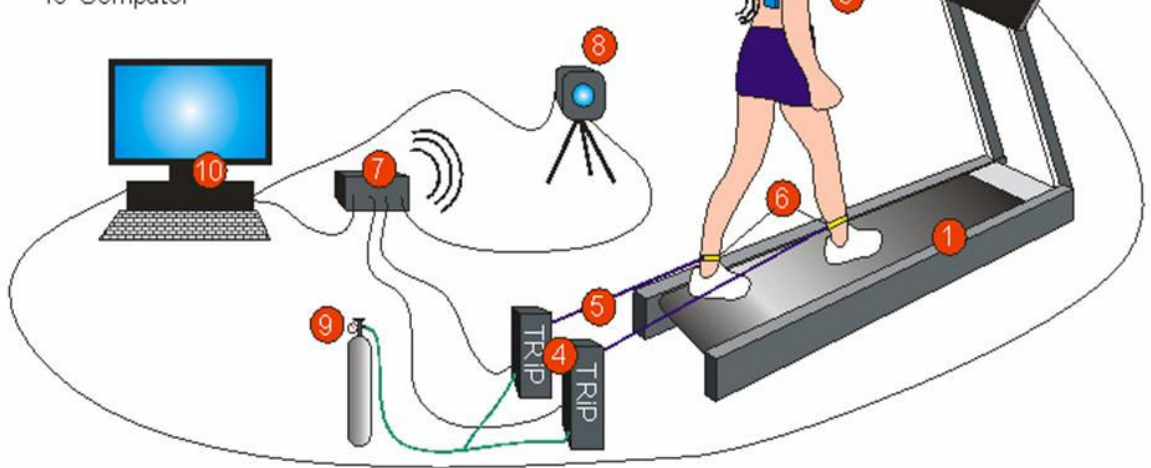

Fig. 1: The Timed Rapid Impact Perturbation (TRiP) set-up, used to evoke controlled perturbations in a standardized way, consists of a treadmill and two trip devices (online supplement).

The triaxial accelerometer shown in this figure is not part of the TRiP set-up. The accelerometer, as presented here, was used to investigate the influence of the trip on gait, which showed to have no effect.

Moreover before the experiment, subjects walked two minutes on the treadmill, first without and subsequently with the TRiP to become accustomed to the set-up. Comparing gait of 5 young and 5 elderly subjects using acceleration-based gait analysis showed no significant differences in gait between overground walking and treadmill walking, neither between treadmill walking with and without the TRiP (results not shown).

\section{2) Muscular factors}

Isometric knee extension and flexion strength was measured using a dynamometer (Biodex III) (14-15). Subjects were seated with their hip and knee in $90^{\circ}$ of flexion and had to produce successively maximal isometric knee extension and flexion strength as fast as possible while maintaining maximal force for 5 seconds. Only the right leg was tested (16). Maximum knee flexion and extension moments and the rate of moment development (RMD), defined as the percentage of the maximum moment attained 200ms after the start of the contraction was determined (14-15). All participants were consistently verbally encouraged. 


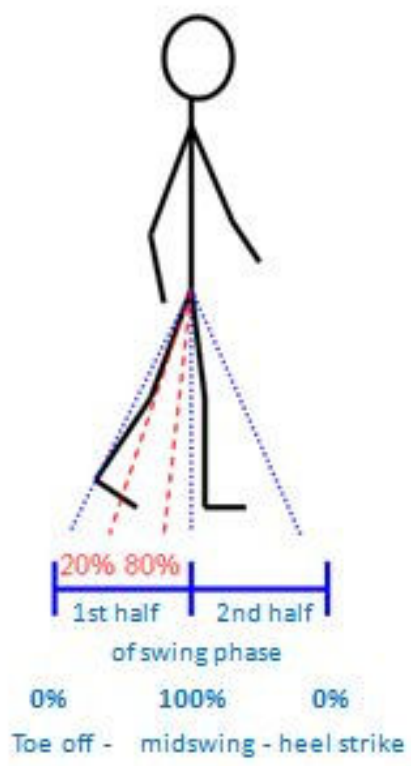

Fig. 2: Perturbations applied during the first half of the swing phase.

The dotted blue lines represent the swing phase of the gait cycle which starts with toe off (0\%), followed by mid swing $(100 \%)$ and ending with heel strike $(0 \%)$. Perturbations were induced during the first half of the swing phase (toe off - mid swing\%); at $20 \%$ (early swing perturbations) and $80 \%$ (mid swing perturbations), as is shown by the red dotted lines

\section{3) speed of information processing factors}

Speed of information processing was determined using a computer-based fourchoice finger-cuing reaction time task (17). Subjects were seated straight behind a computer with the index and middle finger of both hands on specific keys on the bottom row of the keyboard. A row of four squares were continuously visible in red outlines on the computer monitor, while two conditions were presented. Within the uncued condition, all the four squares coloured red, and after an interval of $100,250,500,750$ or $1000 \mathrm{~ms}$, one of these four squares coloured green. In the cued condition, only two out of the four squares (either the two left most or the two right most) coloured red, and after one of the above mentioned intervals, one of the not-indicated squares coloured green. The cue indicated the preparation of the response hand opposite to the location of the cue. Subjects had to respond as quickly and accurately as possible by pressing the key that corresponded to the green coloured square on the screen. At the start of each trial, a warning signal was presented shortly by a small red square midway between the two inner boxes (17). Subjects performed 100 trials in the uncued condition and 100 trials in the cued condition, making a total of 200 trials, randomly mixed. For each subject, the average reaction time over both cue conditions and five time intervals was 
calculated as an index of speed of information processing. An additional parameter was calculated, cognitive flexibility, defined as the ability to prepare the responses indicated by the cue; this was operationalized as the difference in reaction time between the uncued (4-choice; no preparation) and cued (2-choice; advance preparation) condition. A positive value indicated a reaction benefit; a negative value indicated a reaction time cost (17).

\section{STATISTICAL ANALYSIS}

Age differences were investigated using independent t-test or Mann Whitney Utest and the Chi-square test. Pearson's r correlation was performed to investigate correlations between the parameters; recovery response, age, walking speed, speed of information processing, muscle strength parameters. Linear regression analysis (backward method) using recovery response as the outcome measure and muscle strength parameters, speed of information processing parameters, age and walking speed as independent variables, was done to investigate the combined effect of these parameters on the recovery response. All statistics were done in SPSS 15.0, using significance level of 0.05 .

\section{RESULTS}

In total 1900 trips were recorded, of which 34 were missed due to minor technical issues. One reaction time test was missed due to computer problems and four muscle strength measurements were excluded because of measurements errors, as indicated as extreme outliers in a box plot. Further analyses were based on 48 young and 43 elderly subjects.

Elderly subjects were significantly weaker, produced lower RMD, had a slower speed of information processing and a lower cognitive flexibility than young subjects. Elderly had also a slower comfortable walking speed than young subjects (Table 1). Both elderly and young subjects employed mainly LS (73\% of all perturbations) to recover from perturbations in gait. Elderly subjects recovered less by ES than young subjects ( $21 \%$ vs. $31 \%$ of the perturbations, $p<0.01$ ). Of the mid swing perturbations $96 \%$ was recovered by LS (young: $94 \%$, elderly: $99 \%, p<0.001$ ) and $50 \%$ of the early swing perturbations was recovered by ES (young: $57 \%$, elderly: $42 \%, p<0.001)$. Eight elderly and four young subjects used at least once the handrails of the treadmill during recovery (30/1900 perturbations). This occurred at the beginning of the experiment. These trials were included in the analysis because the recovery was already started before the hands were used. 
The recovery response correlated weakly with age $(r=-0.23, p<0.05)$ and speed of information processing $(r=-0.24, p<0.05)$ individually, but not with walking speed $(r=-0.04, p>0.05)$, cognitive flexibility $(r=0.05, p>0.05)$ and strength parameters ( $r$ range: $0.29-0.23, p>0.05)$. In combination however, these parameters had an effect on the recovery response: $23 \%$ of the variability in recovery response was explained by a combination of slow walking speed $(B=-13.85)$, fast speed of information processing $(B=-0.08)$, high maximum extension strength $(B=0.05)$ and high RMD-extension $(B=0.19)$. Age and cognitive flexibility were removed from the model. Strong correlations were found between knee extension and flexion strength parameters ( $r$-range: 0.6-0.8). Therefore, only the extension strength parameters were included in the model. The strength in extensor muscles was preferred because the extensors play an important role during recovery, especially to provide adequate push-off forces in the support $\operatorname{limb}(8,18)$. Age correlated positively with the speed of information processing $(r=0.82)$ and negatively with maximum extension strength ( $r=-0.45)$, RMD-extension ( $r=-0.38)$, maximum flexion strength ( $r=-0.48)$, RMD flexion ( $r=-0.55)$, cognitive flexibility $(r=-0.41)$ and walking speed $(r=-0.67)$. The walking speed correlated weak to moderate with strength parameters ( $r$-range: $0.45-0.55)$, speed of information processing parameters ( $r$ range: $0.30--0.71)$ and age $(r=0.68)$.

Table 1: Averages, \pm standard deviation [ranges] for subject characteristics, tinetti scores, speed of information processing and strength measures for young and older subjects.

\begin{tabular}{|c|c|c|c|}
\hline & Young $(n=48)$ & Old $(n=43)$ & P-value \\
\hline Age (yrs) & $\begin{array}{l}24.4 \pm 4.0 \\
{[18-36]}\end{array}$ & $\begin{array}{l}67.4 \pm 6.2 \\
{[60-82]}\end{array}$ & $0.00^{*}$ \\
\hline Heigth (m) & $\begin{array}{l}1.76 \pm 0.09 \\
{[1.60-1.94]}\end{array}$ & $\begin{array}{l}1.70 \pm 0.10 \\
{[1.48-1.92]}\end{array}$ & $0.00 *$ \\
\hline Weight (kg) & $\begin{array}{l}69.7 \pm 10.3 \\
{[50.0-92.0]}\end{array}$ & $\begin{array}{l}74.1 \pm 12.2 \\
{[43.0-100.0]}\end{array}$ & 0.64 \\
\hline Tinetti score & $\begin{array}{l}28.0 \pm 0.0 \\
{[28-28]}\end{array}$ & $\begin{array}{l}26.9 \pm 1.4 \\
{[24-28]}\end{array}$ & $<0.01^{*}$ \\
\hline Max. flexion (Nm) & $\begin{array}{l}90.0 \pm 30.1 \\
{[36.6-162.6]}\end{array}$ & $\begin{array}{l}60.6 \pm 22.8 \\
{[24.5-148.2]}\end{array}$ & $<0.01^{*}$ \\
\hline RMD flexion (\%) & $\begin{array}{l}62.8 \pm 19.3 \\
{[25.4-97.7]}\end{array}$ & $\begin{array}{l}39.1 \pm 17.6 \\
{[8.6-81.3]}\end{array}$ & $<0.01^{*}$ \\
\hline Max. extension (Nm) & $\begin{array}{l}232.8 \pm 73.7 \\
{[114.0-392.2]}\end{array}$ & $\begin{array}{l}166.0 . \pm 51.6 \\
{[84.8-270.4]}\end{array}$ & $<0.01^{*}$ \\
\hline RMD extension (\%) & $\begin{array}{l}44.7 \pm 20.3 \\
{[4.5-86.0]}\end{array}$ & $\begin{array}{l}26.8 \pm 21.2 \\
{[4.3-85.4]}\end{array}$ & $<0.01^{*}$ \\
\hline Avg. reaction time (ms) & $\begin{array}{l}360.0 \pm 32.7 \\
{[297.5-437.5]}\end{array}$ & $\begin{array}{l}534.8 \pm 82.1 \\
{[377.9-820.7]}\end{array}$ & $<0.01^{*}$ \\
\hline Cognitive flexibility (ms) & $\begin{array}{l}136.3 \pm 56.9 \\
{[15.0-269.0]}\end{array}$ & $\begin{array}{l}51.9 \pm 136.6 \\
{[-280-370]}\end{array}$ & $<0.01^{*}$ \\
\hline Walking speed (km/h) & $\begin{array}{l}4.9 \pm 0.6 \\
{[3.7-6.0]}\end{array}$ & $\begin{array}{l}3.8 \pm 0.6 \\
{[2.7-5.5]}\end{array}$ & $<0.01^{*}$ \\
\hline $\begin{array}{l}\text { Recovery response } \\
\text { (\% perturbations recovered by ES) }\end{array}$ & 31 & 21 & $<0.01^{*}$ \\
\hline
\end{tabular}




\section{Discussion}

This is the first study were a sizable population $(n=95)$ of young and elderly subjects is exposed to controlled perturbations during walking. The objective was to investigate associations between age, walking speed, speed of information processing, muscle strength and the recovery response to perturbations in gait. The recovery response was expressed as the proportion perturbations recovered by ES. In contrast to the expectation, the recovery response is only weakly associated with walking speed, knee extension strength, rate of extension moment development and speed of information processing, showing in addition no effect of age and cognitive flexibility.

Elderly subjects who are weaker and who react more slowly recovered less frequently by ES compared to the stronger and faster reacting young subjects. This is similar to previous studies showing that elderly less often adopt an ES compared to young subjects $(8-9,11)$ and confirms the hypothesis suggesting that there is a shift from ES to LS with physiological changes. Age, walking speed, strength parameters, speed of information processing and cognitive flexibility have individually no or only a small influence on the recovery response $\left(R^{2}\right.$ range $=0.002$ 0.06). In combination however, their contribution increases although the association with the recovery response remains weak: only $23 \%$ of the variability in recovery response can be explained by fast speed of information processing, high muscle strength and slow walking speed. The fact that these factors are involved in balance recovery corresponds to previous studies showing clear effects of muscular responses, muscle strength, lower limb positioning, coordination parameters, reaction times etc. on walking stability and the success rate to avoid obstacles $(4,8$ $11,19)$. The weak associations however assume that other, currently not measured, factors have a bigger impact or that a combination of multiple factors including e.g. walking speed, speed of information processing, muscle strength related factors and other internal (e.g. coordination (19)) and external (e.g. environment) factors modify the recovery response. The influence of external factors has been investigated in the study of Schillings et al. showing that the final recovery response is adjusted to the demands of the moment, achieved by afferent information during the perturbation (e.g. mechanoreceptor feedback) (4).

Age was excluded from the model, suggesting that the recovery response is indirectly affected by age via its deteriorating effects on physiological factors (e.g. via adjusted muscle strength) (20-21). This is also indicated by the moderate to strong correlations found between age and physiological factors and is supported by the weak correlation observed between the recovery response and age individually.

No (individually) or only a small (regression) effect of comfortable walking speed was found on the recovery response. However walking speed may have an effect. 
Pavol et al. shows that a faster walking speed is associated to falls following a trip in healthy elderly subjects (11). Moreover, a pilot study showed that similar perturbations were less frequently recovered by ES when walking at $6 \mathrm{~km} / \mathrm{h}(20 \%)$ compared to when walking at $3 \mathrm{~km} / \mathrm{h}(77 \%(13))$. This suggests that not walking speed perse, but deviations from the comfortable walking speed (e.g. faster or slower than preferred) may influence the recovery response. This requires further examination.

The strength of the associations may have been mitigated by the fact that the elderly subjects were all healthy subjects, not at risk for falling (Tinetti score $>24$ ) (22). It cannot be excluded that the inclusion of elderly subjects at risk for falling may result in more pronounced effects. This is assumed based on previous studies indicating that elderly with a fall history have a slower reaction time than elderly without a fall history (23) and that elderly fallers are weaker, producing lower joint moments than elderly non-fallers (24-25). Moreover the current study focused only on perturbations induced during the first half of the swing phase. Further insight into the associations between the recovery response to external perturbations in gait and physiological factors can be achieved by including perturbations induced during the second half of the swing phase. In addition it can be argued that a more in-depth evaluation of the underlying biomechanics of the recovery strategies (e.g. hip moments) could attenuate the results, which currently rely on quantitative measures (\%ES). Finally, recovery responses induced by the TRiP set-up may not be representative for the type of perturbation typically experienced by subjects in daily life. The TRiP creates an ankle blockage, while tripping over an obstacle may create an impact at the foot, which may affect the recovery response employed. For instance to cross an obstacle in daily life, the use of a LS may be impeded as subjects avoid to step onto the obstacle. Furthermore, the treadmill may create differences in recovery response compared to overground walking as subjects have to keep up with the speed of the treadmill (6). These are general problems of standardised laboratory-based trip set-ups and experiments. Many factors may play a role in tripping during daily life (e.g. environment) which can only be captured by monitoring tripping reactions in daily life. The TRiP set-up however is a good approach to investigate recovery responses for a specific kind of perturbations as it allows simulating perturbations of different compliance by controlling the duration of the perturbation, the perturbation force and the timing of the obstruction in the swing phase. Moreover qualitative agreement (i.e. comparable recovery responses) was found with studies using different approaches to induce perturbations in gait $(4-6,8-9,11)$.

Previous studies have shown that trip recovery responses may be improved by training (26-27). Therefore it is important to further investigate factors that modify the recovery response, which may include e.g. balance, coordination and reflex 
reactions. This insight may help fall prevention programs aiming to improve recovery responses after gait is perturbed.

\section{CONCLUSION}

The recovery responses that subjects employ when gait is perturbed using the TRiP set-up are moderated by several mediating factors in which the contribution of the comfortable walking speed, muscle strength- and speed of information processing related factors is small. Insight into remaining factors modifying the recovery response is needed to assist and optimise fall prevention programs. 


\section{RefERENCES}

1. Feder G, Cryer C, Donovan S, Carter Y. Guidelines for the prevention of falls in people over 65 years. The Guidelines Dev Group BMJ 2003;321:1007-11.

2. Srygley JM, Herman T, Giladi N, Hausdorff JM. Self-report of missteps in older adults: A valid proxy of fall risk? Arch Phys Med Rehabil 2009;90:786-92.

3. Berg WP, Alessio HM, Mills EM, Tong C. Circumstances and consequences of falls in independent community-dwelling older adults. Age Ageing 1997;26:261-8.

4. Schillings AM, Wezel van BMH, Mulder T, J D. Muscular responses and movement strategies during stumbling over obstacles. J Neurophysiol 2000;83:2093-102.

5. Eng JJ, Winter DA, Patla AE. Strategies for recovery from a trip in early and late swing during human walking. Exp Brain Res 1994;102:339-49.

6. Forner-Cordero A, Koopman HJFM, van der Helm FCT. Multiple-step strategies to recover from stumbling perturbations Gait Posture 2004;18:47-59.

7. Hof AL. The equations of motion for a standing human reveal three mechanisms for balance. J Biomech 2007;40:451-7.

8. Pijnappels $M$, Bobbert MF, van Dieën JH. Push-off reactions in recovery after tripping discriminate young subjects, older non-fallers and older fallers. Gait Posture 2005;21(4):388-94.

9. Roos PE, McGuigan MP, Trewartha G. The role of strategy selection, limb force capacity and limb positioning in successful trip recovery. Clin Biomech 2012;25:873-8.

10. Lamoureaux EL, Sparrow WA, Murphy A, Newton RU. The effects of improved strength on obstacle negotiation in community living older subjects Gait Posture 2003;17:273-83.

11. Pavol MJ, Owings TM, Foley KT, Grabiner MD. Mechanisms leading to a fall from an induced trip in healthy older adults. J Gerontol 2001; 56A(7): M 428-437.

12. Bogert van den AJ, Pavol MJ, MD G. Response time is more important than walking speed for the ability of older adults to avoid a fall after a trip. J Biomech 2002;35:199-205.

13. Senden R, Willems P, Savelberg H, Grimm B, Heyligers IC, Meijer K. Validation of the TRiP, a device for timed rapid induced perturbations during treadmill walking. Proceedings of $13^{\text {th }}$ congress of International Society of Biomechanics (ISB), Brussels, 2011.

14. Aagaard P, Simonsen EB, Andersen JL, Magnusson P, Dyhre-Poulsen P. Increased rate of force development and neural drive of human skeletal muscle following resistance training. J Appl Physiol 2002; 93: 1318-2602.

15. Andersen LA, Aagaard P. Influence of maximal muscle strength and instrinsic muscle contractile properties on contractile rate of force development. Eur J Appl Physiol 2006; 96: 46-52.

16. Hageman P, Gillespie D, Hill L. Effect of speed and limb dominance on eccentric and concentric isokinetic testing of the knee. J Orthop Sports Phys Ther 1988;10:59-65.

17. Adam JJ, van Houdt H, Scholtissen B, VIsser-Vandewall V, Winogrodzka A, Duits A. Executive control in Parkinson's disease: Effects of dopaminergic medication and deep brain stimulation on anti-cue keypress performance. Neuroscience Letters 2011:113-7.

18. Pijnappels M, Reeves ND, Maganaris CN, Dieën van JH. Tripping without falling: lower limb strength, a limitation for balance recovery and a target for training in the elderly $\mathrm{J}$ electromyography and kinesiology 2008;18:1880196.

19. Krasovsky T, Banina MC, Hacmon R, Feldman AG, Lamontagne A, Levin MF. Stability of gait and interlimb coordination in older adults. J Neurophysiol 2012; 107(9):2560-2569.

20. Menz HB, Lord SR, Fitzpatrick RC. Acceleration patterns of the head and pelvis when walking are associated with risk of falling in community-dwelling older people. J Gerontol 2003;58a:446-52.

21. Skeleton DA, Greig CA, Davies JM, Young A. Strength, power, and related functional ability of healthy people aged 65-89years,. Age Ageing 1995;23:371-7.

22. Tinetti ME. Performance-oriented assessment of mobility problems in elderly patients. J Am Geriatr Soc 1986;34:119-26.

23. Grabiner $\mathrm{MD}$, Jahnigen DW. Modelling recovery from stumbles: preliminary data on variable selection and classification efficacy. J of Am Ger Soc 1992;40:910-3.

24. Simoneau DD, Krebs DE. Whole-body momentum during gait: A preliminary study of non-fallers and frequent fallers. Journal of Applied Biomech 2000;16(1):1-13.

25. Spiduso WW, ed. Physical dimensions of aging. Champaign: Leeds: Human Kinetics, 1995. 
26. Bieryla KA, Madigan ML, Nussbaum MA. Practicing recovery from a simulated trip improves recovery kinematics after an actual trip. Gait Posture 2007;26:208-13.

27. Pavol MJ, Runtz EF, Pai YC. Young and older adults exhibit proactive and reactive adaptations to repeated slip exposures. J Gerontol A-Biol 2004;59:494-502. 



\section{Chapter 9 \\ General Discussion}


The aim of this thesis is to evaluate the suitability of an acceleration-based gait analysis system that relies on a single triaxial accelerometer measuring accelerations at the level of the sacrum (AGA) for describing gait of various subject groups such as healthy young and older subjects, patients with knee limitations and elderly people at risk of falling. Moreover fall risk is investigated in more detail beyond acceleration-based gait analysis by focussing on perturbations in gait. Insight into the causal factors behind a fall (e.g. recovery response employed after being tripped) is required to understand why individuals fall and why they are at risk for falling.

This thesis has addressed three research questions, which have lead to the study aims explored in the individual chapters:

1. Can acceleration-based gait analysis be used to create a reliable reference gait database for healthy subjects? (chapters 2-3)

2. Is acceleration-based gait analysis of clinical use for patients with functional limitations of the lower extremities? (chapters 4-5)

3. Are gait parameters measured by acceleration-based gait analysis, and the recovery responses to external perturbations in gait, associated with fall risk? (chapters 6-8)

The three research questions are discussed individually in this general discussion, together with improvements and recommendations for future studies. Finally a general conclusion is given.

\section{CAN ACCELERATION-bASEd GAIt ANALYSIS BE USED to CREATE A RELIABLE REFERENCE GAIT DATABASE FOR HEALTHY SUBJECTS?}

In clinical practice, there is a need for more objective and precise functional assessment tools. Acceleration-based gait analysis has been shown to have potential, but information about its reliability has been lacking. In addition, no reference database on AGA-parameters is available. The first part of this thesis therefore investigates whether AGA can be used to create a reliable reference gait database for healthy subjects. And the answer is simply 'yes'. Spatio-temporal gait parameters are reliably derived from the acceleration signal (1-2). This indicates that AGA is robust and unaffected by differences in performance techniques used by different observers, which is a requirement for clinical use where different observers will perform the test. AGA is also sensitive to subject characteristics, showing for instance typical effects of gender, age and body height such as smaller step frequency in men, slower speed in the elderly and longer steps in tall subjects $(1,3)$. The fact that AGA is able to detect subtle differences in gait due to subject characteristics implies that AGA will also be sensitive to pathologies, which in general concur with more serious gait deviations. The clinically important, 
independent and non-linear effects of age, gender and body height on gait indicate that a reference gait database is needed in order to apply AGA in clinical practice (3). Such a database for healthy subjects with varying subject characteristics has been created based on AGA (1). This database provides insight into the norm values of gait parameters essential for evaluating pathologies and therapeutic interventions. The database also allows the identification of gait changes at specific time points throughout a person's lifespan, which enables prompt tracking of abnormalities. A further extension of the reference database, in particular the inclusion of more small and tall subjects and more younger $(<20 \mathrm{yrs}$ ) and older (>70 yrs) subjects, is recommended to get further insight into the distribution of gait parameters according to age, gender and body height. Gait analysis of slim and overweight subjects will provide more insight into the influence of body mass on gait. Moreover, other gait parameters as described in chapter 6, such as the harmonic ratio as a measure of smoothness of walking and inter-stride amplitude variability as a measure of gait variability, can be added to the reference database (4). The extended database will further improve the interpretation of gait and thus contribute to improved clinical diagnostics and follow-up capabilities. Recently Schwesig et. al. has presented a reference data on spatio-temporal gait parameters of men and women of all ages (range 5 - 100 yrs) using an inertial sensor based gait analysis system (5). This study confirms the importance of, and clinical need for, a reference gait database.

Besides expanding the database, further improvements can be made, especially concerning the calculation of step time asymmetry and gait irregularity. Currently the calculation of step time asymmetry and gait irregularity relies on exact heel strike (peak) timing and is therefore very sensitive to inaccurate peak detections (e.g. shifted, double or missed peaks) (6-7). This contrasts with the spatio-temporal gait parameters, which rely on step counting. Optimising the peak detection algorithm, for instance by using new techniques (e.g. spectrogram, template method) may improve the accuracy and reliability with which step time asymmetry and irregularity are calculated (7). Alternative parameter definitions may also be used. For instance, gait irregularity could be determined using autocorrelations or by coefficient of variation as done in other studies (8-9).

\section{IS ACCELERATION-BASED GAIT ANALYSIS OF CLINICAL USE FOR PATIENTS WITH FUNCTIONAL LIMITATIONS IN THE LOWER EXTREMITIES?}

The reliability of AGA in describing human gait, and the sensitivity of AGA in detecting changes in gait due to subject characteristics, suggest that AGA may be suitable for clinical use. However, to be used in clinical practice AGA also needs to have discriminative power to ensure that abnormalities from healthy gait are 
detected. In addition, AGA needs to be able to measure changes over time in a repeatable way. Differences in AGA-parameters are found between healthy subjects (reference database) and subjects suffering functional knee limitations (e.g. simulated arthrodesis (2), osteoarthritis (10)). This shows the discriminative power of AGA to differentiate subjects with knee limitations from healthy subjects (2). AGA is also sensitive for different knee-limited walking conditions, showing increasing deviations in AGA-parameters with increasing severity of the knee limitation. The deviations in gait are measured in a repeatable way (2) and are comparable to other studies using laboratory-based motion analysis systems (1114). These findings indicate that $A G A$ is sensitive to different functional knee limitations and is reliable for identifying deviations in gait. Moreover, AGA detects subtle changes in gait due to intervention (e.g. total knee arthroplasty, TKA (10)) and rehabilitation (10) indicating that AGA can be used to monitor patients with knee limitations over time, for instance during their functional recovery. All these findings together demonstrate the clinical suitability of AGA for subjects with functional knee limitations. The fact that subtle (e.g. change in step length of $1 \mathrm{~cm}$ ) deviations in gait are identified by AGA, and that AGA is sensitive to the seriousness of functional knee limitation suggest that AGA will also be sensitive to other pathologies (e.g. hip limitations, ankle problems). Future studies investigating the limits of detection by AGA (what's the smallest detectable deviation?) and examining gait of different patient groups are needed to determine this.

The walking speed shows the highest discriminative power in differentiating subjects with knee limitations from healthy subjects. This is not surprising as walking speed has previously been associated with health and well-being (15-16). However, walking speed is an a-specific outcome parameter as it has been related to various pathologies and diseases (e.g. Parkinson's' disease (17)). For clinical purposes a more in depth evaluation of the gait pattern, beyond walking speed, is therefore recommended. AGA produces several gait parameters simultaneously, allowing extended and detailed insight into the gait pattern of individuals. This, together with the finding that AGA measures different aspects of function than the standard clinical scales (e.g. Knee Society Score) (10) shows the additional benefits of AGA in identifying functional limitations and monitoring patients in clinical practice. A combined use of standard clinical scales (measuring pain and emotionally-related aspects of function) with AGA (measuring objective gait parameters) is recommended to achieve a more complete overview of a patients' clinical state, as recently supported by Liebensteiner et. al. (18).

An optimal patient evaluation is necessary in current clinical practice because function has become increasingly important for the new generation of patients, who are younger and more demanding. In order to achieve optimal functional levels after surgery, it is important to supervise the recovery process carefully. This requires frequent and accurate function evaluation in an objective and 
individualised way. This can be achieved by AGA, which shows the importance of AGA to the clinical practice. For instance, AGA allows prompt identification of small changes in gait (improvements and impairments), which may result in optimal patient-specific treatments. Moreover frequent function evaluation by AGA may provide feedback to the patient and therapist, which may optimise the recovery process.

In order to be applied in clinical practice AGA must be practical and user-friendly. The single triaxial accelerometer is small, lightweight, portable and can easily be attached to, and removed from, the body enabling its use in routine clinical practice. In addition, the sensor does not impede or influence gait pattern and its use is risk free. The user-comfort of AGA, together with its ability to describe human gait, demonstrates the suitability of AGA for clinical use. AGA can be applied as an objective, functional assessment tool to evaluate interventions (10), to objectively monitor the function of patients during follow up (10) and to identify deviating gait patterns $(2,10)$.

\section{ARE GAIT PARAMETERS MEASURED BY ACCELERATION-BASED GAIT ANALYSIS AND THE RECOVERY RESPONSES TO EXTERNAL PERTURBATIONS IN GAIT ASSOCIATED TO FALL RISK?}

Changes in gait, such as a higher stride-to-stride variability, have been identified as factors in fall risk. In clinical practice, fall risk is frequently quantified by visually observing functional limitations in gait (19-22). This suggests that objective gait analysis by AGA may be a valuable additional tool in fall risk screening (4). Differences in AGA parameters are observed in elderly subjects with different scores on the Tinetti scale (4), which are comparable to fall risk related gait characteristics reported elsewhere (19-22). This indicates that AGA captures fall risk related gait characteristics and shows the potential of AGA as objective gait analysis system in elderly at risk for falling. Walking speed, step length and preferably the Root Mean Square (RMS, derived from vertical acceleration signal) can best be used to classify elderly according to the Tinetti score, as these parameters show the highest discriminative capacity $(4,22)$. The poor to moderate correlations found between the Tinetti scale and gait parameters that have frequently been considered indicators for an elevated fall risk (e.g. harmonic ratio, inter-stride amplitude variability $(4,20,21,23)$ indicate that not all fall risk related gait characteristics are captured by subjective assessments of fall risk, in this case by the Tinetti scale. This shows the additional value of AGA beyond the commonly used subjective assessment and indicates that it is worthwhile to include objective gait monitoring in fall risk screening, which can easily be done by AGA. Besides fall 
risk screening, AGA may be relevant for fall prevention programs, as it provides detailed and objective information about changes and deviations in gait $(4,10,24)$. Previous studies have shown that exercise (e.g. Tai Chi, 25) and other interventions may be effective in minimizing fall risk (26-27). The objectively measured fall risk related gait characteristics may yield targets for fall prevention programs. AGA may help to make decisions regarding fall prevention and may allow to evaluate, optimize and develop (patient specific) fall prevention programs (e.g. gait training). Insights into risk factors for falling allow us to quantify fall risk, but they do not explain why individuals fall. This requires a more in-depth evaluation of the causal factors of a fall. The final part of this thesis explores human stability during walking and focuses on recovery to external perturbations in gait, which account for more than half of the falls in elderly (28). For this purpose a trip set-up (the Timed Rapid impact Perturbation, TRiP) is designed able to induce controlled perturbations of varying characteristics (duration, force and timing) with moderate to good accuracy. In addition the TRiP elicits the two stereotypical recovery strategies described in literature (the lowering strategy and elevating strategy (29-32)) (33). Subsequently, the TRiP is used to explore recovery responses to external perturbations in gait in a large group of healthy young and elderly subjects. Previous studies have shown that physiological factors such as muscle strength clearly affect walking stability and adequate trip recovery $(29,30,33)$. This suggest that these factors also contribute to the recovery response (elevating vs. lowering strategy) employed by individuals when gait is perturbed. Accordingly, the older subjects who are weaker, who react more slowly and who walked more slowly employ mainly the secure lowering strategy, while the fast walking, stronger and fast reacting young subjects employ more often the more demanding elevating strategy. This suggests that there is a shift from elevating strategy to lowering strategy with age-related physiological changes. However walking speed, muscle strength and speed of information processing are only weakly associated with the recovery response (34). This indicates that additional factors are modifying the recovery response employed when gait is perturbed by the TRiP set-up. Although qualitative agreement (e.g. comparable recovery strategies, faster switch to lowering strategy in the elderly) is found with previous studies using different setups (e.g. treadmill vs. overground walking, tripping leashes vs. obstacle), the observed associations are weaker than expected from previous studies. It can be argued that individuals take account of the situation in which they are perturbed. For instance, individuals apply elevating and lowering strategies when gait is perturbed in early swing (10-20\% of total swing phase) using the TRiP set-up while only elevating strategies are observed in studies using a different set-up $(29,31$, 35). To answer the question whether recovery responses to external perturbations in gait are associated to fall risk, more research is needed including the exploration of associations between recovery responses, physiological changes and external 
factors (e.g. the condition in which gait is perturbed). This may require the inclusion of elderly subjects having more serious physiological impairments. In addition more investigation is needed to examine the remaining factors modifying the recovery response (e.g. coordination, reflexes). This insight is needed to assist and optimise fall prevention programs aimed at reducing fall risk by improving recovery responses to perturbations in gait $(34,36-37)$.

\section{RECOMMENDATIONS FOR FUTURE STUDIES}

The clinical suitability of AGA for identifying deviations in gait $(2,4,10)$, evaluating interventions and assessing the functional recovery of patients (10) has been demonstrated in this thesis. AGA may, however, serve additional clinical purposes. AGA may help in clinical diagnostics, and it may serve to evaluate and compare surgical approaches and implant types. It may also assist in clinical decision making, as it provides objective and detailed information about a subject's gait performance. It may be useful in evaluating rehabilitation programs and to improve or develop new therapies. If AGA is applied routinely in clinical practice it may serve as a feedback system for patients, clinicians and clinical researchers. A regular evaluation of a patient's function using AGA may also give rise to patient specific treatments. All this may finally result in improved and individualised patient care. However, to use AGA for these clinical purposes more research is needed.

To use AGA for clinical diagnostics (e.g. to diagnose meniscal tear), improvements need to be made. A pilot study has shown that AGA lacks the discriminative power to distinguish different pathologies (e.g. TKA vs. meniscal tear). A more advanced technique, including a more challenging functional task (e.g. stair climbing (36-39)), another inertia sensor (accelerometer \& gyrometer) and/or additional gait parameters (e.g. pelvic obliquity (40)) is recommended for this purpose. Further studies are needed to establish the reliability and validity of this advanced technique.

In addition, the clinical implementation of AGA may be limited by two practical issues. The tests are currently performed on a $20 \mathrm{~m}$ undisturbed walkway, which may be difficult to find in hospital settings. In addition, the exact distance has to be measured with a ruler, which means extra work for the observer. Measuring gait in ordinary living conditions may solve these practical issues. In addition, field measurements will improve the quality of the data. A more representative and natural gait pattern will be obtained in a natural environment, as patients do not feel observed (e.g. no instructions). Moreover, the application of AGA in the field may allow individualised patient care at a distance, which may improve clinical diagnostics and evaluations while reducing costs and the demands on patient and 
clinician (e.g. less time, no travelling). However, in order to use AGA in ordinary living conditions for clinical purposes, more research is required. First of all, the ability of AGA to accurately and reliably analyse gait in ordinary living conditions needs to be investigated. Algorithms need to be adjusted and factors that may influence the AGA measurement such as for instance the environment and the walking surface (e.g. sand, loose stones), need to be investigated.

Another recommendation for future studies is to investigate the clinical feasibility of AGA in other patient groups such as patients with neurological complaints or patients with pulmonary diseases. In this way other clinical departments can profit from the benefits of AGA. The reference database of healthy subjects can be used to evaluate gait of these patients.

In addition AGA may play a role in future studies into falls and fall risk. A pilot study using the TRiP to induce controlled perturbations under standardized conditions, has shown that a single triaxial accelerometer attached at the sacrum of the subject is able to detect perturbations in gait with a sensitivity of $98.4 \%$ and a specificity of $99 \%$ (41). These results are promising, however the observed false positives and false negatives indicate that further improvements are needed before it can successfully be applied in non-standardised conditions such as in daily life.

If AGA is able to detect perturbations in gait in uncontrolled conditions, AGA could be used for several scientific issues. For instance to examine whether the increased fall incidence in the elderly population is associated with an increased trip incidence. This is still an unknown aspect, but very relevant for fall prevention programs. In addition, the detection of perturbations in gait during daily life may allow further exploration of recovery responses. The choice for the recovery response can then be associated to more factors, including external factors such as the circumstance in which the perturbation occurs. Moreover it can be investigated whether one recovery response is more successful than another response for certain individuals under certain conditions. This insight could subsequently be applied in fall prevention programs, e.g. in training subjects to employ the most successful recovery strategy. All this information may provide more insight into how recovery responses are associated with falls and fall risk. Further investigation and optimisation of the use of AGA in identifying perturbations in gait in daily life is thus recommended to realize these purposes.

Examination is also recommended into whether a successful recovery response to external perturbations can be learned. During the experiments with the TRiP, it is noticed that individuals have most difficulty in recovering from the first perturbations. This is exhibited as the use of hands or an obvious scared reaction. At the end of the experiments, perturbations are more easily recovered $(31,35)$. If a successful recovery can be trained, the TRiP set-up could be useful for fall prevention programs aimed at optimising recovery responses in order to reduce fall incidence. 


\section{General Conclusion}

Human gait analysis by a single triaxial accelerometer measuring accelerations at the level of the sacrum (AGA) is reliable and sensitive to subject characteristics (e.g. age, gender, body height) and health-related issues (e.g. osteoarthritis and fall risk). AGA can be applied to several subject groups providing an objective and detailed assessment of an individual's gait pattern, showing the additional benefits of AGA for clinical use. This together with the practicality of AGA recommends the implementation of AGA in clinical practice. The database of healthy subjects serves as a reference.

The newly developed and validated TRiP set-up allows further exploration of stability during walking in humans, and has so far shown that the recovery response that subjects employ when gait is perturbed by the TRiP set-up is modified by a combination of multiple factors including muscle strength, speed of information processing and walking speed. 


\section{REFERENCES}

1. Senden R, Grimm B, Heyligers IC, Savelberg HHCM, Meijer K. An acceleration-based gait test for healthy subjects: Reliability and reference data. Gait Posture 2009;30(2):192-6.

2. Senden R, Heyligers IC, Meijer K, Savelberg HHCM, Grimm B. Acceleration-based motion analysis as a tool for rehabilitation: exploration in simulated functional knee limited walking conditions. Am J Phys Med Rehab 2010;90(3):226-32.

3. Senden R, Meijer K, Heyligers IC, Savelberg HHCM, Grimm B. The importance of correcting for individual differences in the clinical diagnosis of gait disorders. Physiotherapy 2012;98:325-329

4. Senden R, Savelberg HHCM, Grimm B, Heyligers IC, Meijer K. Accelerometry-based gait analysis, an addition objective approach to screen subjects at risk for falling. Gait Posture 2012; 36(2):296-300.

5. Schwesig R, Leuchte S, Fischer D, Ullmann R, Kluttig A. Inertial sensor based reference gait data for healthy subjects. Gait Posture 2011;33:673-8.

6. Senden R, Bolink S, Heyligers IC, Meijer K, Savelberg HHCM, Grimm B. How to derive gait parameters from an acceleration-based gait test? International Society of Biomechanics (ISB), Brussels, Belgium, 2011.

7. Senden R, Meijer K, Heyligers IC, Savelberg H, Tilburgh K, Schoeberichts S, et al. Which is the best way to analyze an acceleration-based gait test for routine clinical follow up? European Orthopaedic Research Society (EORS), Davos, Swiss, 2010.

8. Hartman A, Murer K, Bie de RA, Bruin de ED. Reproducibility of spatio temporal gait parameters under different conditions in older adults using a trunk triaxial accelerometer system. Gait Posture 2009;30:351-5.

9. Moe-Nilssen R, Helbostad JL. Estimation of gait cycle characteristics by trunk accelerometry. J Biomech 2004;37:121-6.

10. Senden R, Grimm B, Meijer K, Savelberg H, Heyligers IC. The importance to including objective functional outcomes in the clinical follow up of total knee arthroplasty patients. The Knee 2010;18(5):306-11.

11. Bolanos AA, Colizaza WA, McCann PD, al e. A comparison of isokinetic strength testing and gait analysis in patients with posterior cruciate-retaining and substituting knee arthroplasties. J Arthroplasty 1998;13:906-15.

12. Solak AS, Kentel B, Ates Y. Does bilateral total knee arthroplasty affect gait in women? J Arthroplasty 2005;20:745-50.

13. Fuchs S, Tieck CO, Genkinger $\mathrm{M}$, al e. Clinical and functional comparison of bicondylar sledge prostheses retaining all ligaments and constrained total knee replacement. Clin Biomech 2004;19:263-9.

14. Ivarsson I, Gillquist J. Rehabilitation after high tibial osteotomy and unicompartmental arthroplasty. A comparative study. Clin Orthop 1991;266:139-44.

15. Studenski S, Perera S, Patel K, Rosano C, Faulkner K, Inzitari M, et al. Gait Speed and Survival in Older Adults. JAMA 2011;305(1):50-8.

16. Dumurgier J, Elbaz A, Ducimetière $P$, Tavernier B, Alpérovitch A, Tzourio C. Slow walking speed and cardiovascular death in well functioning older adults: prospective cohort study. BMJ 2009;339:b4460-7.

17. Latt MD, Menz HB, Fung VS, Lord SR. Acceleration patterns of the head and pelvis during gait in older people with Parkinsons' disease: a comparison of fallers and nonfallers. J Gerontol: Med Sciences 2009;64A(6):700-6.

18. Liebensteiner MC, Herten A, MGstoettner AH, Thaler M, Krismer M, Back CM. Correlation between objective gait parameters and subjective score measurements before and after total knee arthroplasty. The Knee 2008;15:461-6.

19. Verghese J, Holtzer R, Lipton RB, Wang C. Quantitative gait markers and incident fall risk in older adults. J Gerontol A Biol Sci Med Sci 2009;64a:896-901.

20. Menz HB, Lord SR, Fitzpatrick RC. Acceleration patterns of the head and pelvis when walking are associated with risk of falling in community-dwelling older people. J Gerontology 2003;58A(5):44652. 
21. Lamoth CJ, Deudekom van FJ, Campen van JP, Appels BA, Vries de OJ, Pijnappels M. Gait stability and variability measures show effects of impaired cognition and dual tasking in frail people. J NeuroEngineering and Rehabilitation 2011;8(2).

22. Bautmans I, Jansen B, Keymolen van B, Mets T. Reliability and clinical correlates of 3Daccelerometry based gait analysis outcomes according to age and fall-risk. Gait Posture 2011;33(3):366-372.

23. Hausdorff JM, Rios DA, Edelberg HK. Gait variability and fall risk in community-living older adults: a 1 year prospective study. Arch Phys Med Rehabil 2001;82:1050-6.

24. Senden R, Heyligers IC, Meijer K, Savelberg HHCM, Grimm B. Acceleration-based motion analysis as a tool for rehabilitation: Exploration in simulated functional knee limited walking conditions. Am J Phys Med \& Rehab 2011; 90(3): 226-32.

25. Wolfs SL, Barnhart HX, Kutner NG, McNeely E, Coogler C, Xu T. Reducing frailty and falls in older persons: an intervention of Tai Chi and computerized balance training. Atlanta FICSIT Group. Frailty and Injuries: Cooperative studies of intervention techniques. J Am Geriatr Soc 1996; 44: 489-97.

26. Province MA, Hadley EC, Hornbook MC, Lipsifz LA, Miller JP, Mulrow CD, et al. The effects of exercise on falls in elderly patients. A prepanned meta-analysis of the FICSIT Trials. Frailty and Injuries: Cooperative studies of intervention techniques. JAMA 1995; 273: 1341-7.

27. Tinetti ME, Baker DI, McAvay G, Claus EB, Garrett P, Gottschalk M, et al. A multifactorial intervention to reduce the risk of falling among elderly people living in the community. $\mathrm{N}$ Enl J Med 1994; 331: 821-7.

28. Srygley JM, Herman T, Giladi N, Hausdorff JM. Self-report of missteps in older adults: A valid proxy of fall risk? Arch Phys Med Rehabil 2009; 692;90:786.

29. Schillings AM, Mulder T, Duysens J. Stumbling over obstacles in older adults compared to young adults. J Neurophysiol 2005;94:1158-68.

30. Pijnappels M. Push-off reactions in recovery after tripping discriminate young subjects, older nonfallers and older fallers. Gait Posture 2005;21(4):388-94.

31. Senden R, Savelberg HHCM, Willems P, Grimm B, Heyligers IC, Meijer K. The TRiP, a specially designed experimental set-up to induce perturbations in a standardised ways: A technical note. To be submitted.

32. Weerdersteyn V, Schillings AM, van Galen GP, Duysens J. Distraction affects the performance of obstacle avoidance during walking. J Mot Behav 2003;35:53-63.

33. Pavol MJ, Owings TM, Foley KT, Grabiner MD. Influence of lower extremity strength of healthy older adults on the outcome of an induced trip. JAGS 2002;50(2):256-62.

34. Forner-Cordero A, Koopman HJFM, Helm van der FCT. Multiple-step strategies to recover from stumbling perturbations. Gait Posture 2004;18:47-59.

35. Senden R, Savelberg HHCM, Adam JJ, Grimm B, Heyligers IC, Meijer K. The influenceof age, muscle strength and cognitive ability on recovery responses to external perturbations in gait. Submitted.

36. Verlaan L, R S, Storken G, Heyligers IC, Grimm B. Outcome assessment in orthopaedics using a stair climbing test and accelerometer based motion analysis: A feasibility study. European Orthopaedic Research Society EORS, Madrid, Spain. 2008.

37. Bolink SAAN, Schoeberichts S, Heyligers IC, Grimm B. Functional scoring of knee patients using a Stand-Sit and a Step-up test with an inertia sensor. Combined Meeting of the Orthopaedic Research Society (CORS), Kyoto, Japan. 2010.

38. Vanlommel L, Schoeberichts S, Heyligers IC, Grimm B. Inertia based analysis of trunk motion during stair test for outcome scoring of knee pathologies. Combined Meeting of the Orthopaedic Research Society (CORS), Kyoto, Japan. 2010.

39. Grimm B, Storken G, Senden R, Heyligers IC. Inertia based motion analysis of a sit-stand-sit test: Suitability as an outcome measure in orthopaedics. Orthopaedic Research Society (ORS), New Orleans, LA, USA, 2009.

40. Bolink SAAN, Brunton L, Blom B, Heyligers IC, Grimm B. Validating an inertial-sensor based gait test and motion parameters for functional outcome scoring in routine clinical practice. International Society for Technology in Arthroplasty (ISTA), Bruges, Belgium 2011.

41. Karel JMH, Senden R, Janssen JEM, Savelberg HHCM, Grimm B, Heyligers IC, et al. Towards unobtrusive in vivo monitoring of patients prone to falling. Conf Proc IEEE Eng Med Biol Soc 2010;1:5018-21. 

Summary 
Gait is a very economical way of human locomotion which has been associated with well- being, health and quality of life. In clinical and research settings, gait performance is widely accepted as a general measure of functional ability among individuals. Human gait analysis using advanced motion capture systems is accurate but not practical for clinical use, and gait assessment by clinical scales is easy and quick, but lacks discriminative power. This shows the need for more objective and more precise clinical functional assessment tools. Accelerometer based motion analysis has been applied to overcome the restrictions of laboratory-based movement systems (e.g. lab dependent) and the limitations of the clinical scales (e.g. subjectivity). Given the promising results of divers accelerometer configurations in analysing gait, and the practical benefits of a single triaxial accelerometer, gait analysis by an acceleration-based gait analysis system measuring accelerations in 3 directions at the level of the sacrum (AGA) may be suitable for clinical use. However, to use AGA in clinical practice a thorough evaluation of its performance in different populations and settings is needed and a reference database of gait in healthy subjects is required. The aim of this thesis is to evaluate the suitability of an acceleration-based gait analysis system that relies on a single triaxial accelerometer measuring accelerations at the level of the sacrum (AGA) for describing gait of various subject groups, including healthy young and older subjects, orthopaedic knee patients and elderly people at risk of falling. In addition, fall risk is investigated in more detail because gait analysis does not provide insight into the causal factors behind a fall necessary to understand better why people fall. The last part of this thesis therefore concentrates on perturbations in gait.

This thesis focuses on three research questions. The first question "Can acceleration-based gait analysis be used to create a reliable reference gait database for healthy subjects?" is investigated in Chapters $2 \& 3$. Secondly, the research question "Is acceleration-based gait analysis of clinical use for patients with functional limitations in the lower extremities?" is examined in Chapters 4 \& 5. Finally the third research question "Are gait parameters measured by accelerationbased gait analysis and the recovery response to external perturbations in gait associated with fall risk?" is studied in Chapters 6, 7 and 8.

Chapter 2 aims to create a reference database of gait in healthy subjects based on AGA-parameters, and examines the reliability of AGA for describing gait. The gait pattern of a large group of healthy subjects of varying subject characteristics is therefore measured by AGA. It is shown that spatio-temporal gait parameters such as walking speed and cadence are measured by AGA with high reliability. Other known clinically relevant gait parameters such as step time asymmetry and gait irregularity are determined with fair to good reliability. In addition the spatiotemporal AGA-parameters show general comparability with basic parameters produced by validated advanced motion capture systems. These findings make AGA 
suitable for gait analysis outside laboratory settings, such as in clinical settings. Moreover, these results indicate that AGA is robust and unaffected by differences in performance techniques used by different observers. This is an important feature for clinical use where different observers have to conduct the gait test on different occasions. A healthy gait database based on AGA-parameters has been created for reference. This reference database allows the comparison of specific gait parameters with groups matched for age, gender and body height, enabling gait of patients to be put in perspective quickly. In addition, insight into the effects of variations in subject characteristics (age, gender, body height and body mass) on various AGA-parameters is needed to interpret gait correctly. This is explored and quantified in Chapter 3 using the reference database created. Clinically important relationships are found between age, body height, gender and spatio-temporal gait parameters. These subject characteristics account for $34 \%$ to $51 \%$ of the variability in spatio-temporal gait parameters. Gait irregularity and step time asymmetry are less influenced by subject characteristics $(<7 \%)$ suggesting that they are more likely to be related to pathologies. Typical correlations are observed between subject characteristics and gait parameters such as the slower walking speed of elderly compared to young subjects and the longer steps of men compared to women. These findings indicate that gait is variable between subjects, showing the importance of a reference database of gait in healthy subjects. From Chapters 2 and 3 it is concluded that AGA is able to describe gait of healthy subjects in a reliable way. The database on AGA-parameters of healthy subjects can serve as a reference for clinical evaluations.

A simulation study is performed to evaluate whether AGA is able to differentiate between different gait conditions in healthy subjects (Chapter 4). Gait of healthy subjects who walked with four different functional knee limitations is therefore analysed by AGA. The different walking conditions are differentiated by AGA. Acute gait changes are detected with high repeatability, showing bigger changes in gait as the severity of the limitation increases. Only gait irregularity is produced with lower repeatability. This may point to variations in gait irregularity measured over several trials or to the fact that AGA is less able to give repeatable results about gait irregularity (e.g. due to systematic errors, device limitations, sensitivity to errors). The changes in gait that are identified by AGA are comparable with previous studies analysing gait of knee patients using laboratory-based motion analysis systems. A first clinical validation study of AGA is done in Chapter $\mathbf{5}$ in which the suitability of AGA for assessing function in orthopaedic patients with symptomatic gonarthrosis indicated for total knee arthroplasty (TKA) is evaluated. AGA is used to describe the gait pattern of patients before and after surgery. In addition, gait of patients is compared to gait of healthy subjects and AGA is compared to current clinical scales (e.g. Knee Society Score). Acceleration-derived spatio-temporal gait parameters show good discriminative power in differentiating gait of knee patients 
from gait of healthy subjects, with walking speed being the most discriminative parameter. In addition, AGA is sensitive to subtle changes in gait measured during recovery after TKA. AGA-parameters show a faster recovery with regards to the clinical scales. Moreover none, or only weak, correlations are found between AGA and clinical scales. This indicates that AGA provides additional information about a patient's function beyond the clinical scales. Chapters 4 and 5 show that AGA is sensitive to different functional knee limitations (e.g. several simulated knee limitations and knee osteoarthritis) and indicate that AGA is able to identify small and unobservable changes in gait over time. It is concluded that AGA is suitable for clinical use. In clinical practice AGA can be used for several purposes, such as to indicate symptoms (e.g. osteoarthritis), to evaluate interventions (e.g. TKA) and to monitor function of patients over time.

Chapter 6 evaluates associations between the Tinetti scale, a correlate for fall risk, and AGA-parameters. The Tinetti scale is completed by elderly subjects, whose gait is evaluated using AGA. Differences in gait are found in elderly subjects with different Tinetti scores. All AGA-parameters correlate with the Tinetti scale. However only strong correlations are found for walking speed, step length and root mean square of the vertical acceleration which also show good discriminative power in classifying elderly subjects according to the Tinetti scale. This shows that AGA captures fall risk related gait characteristics and indicates that AGAparameters are associated with fall risk. However harmonic ratio and amplitude variability, which have been related to fall risk previously, are only poorly to moderately associated with the Tinetti scale. This indicates that not all small differences in gait are captured by subjective fall risk assessments, demonstrating the relevance of including objective gait assessment in fall risk screening. Chapters 7 and 8 examine the causal factors of a fall in more detail and focus on perturbations in gait. To explore walking stability in humans, a laboratory-based TRiP set-up (Timed Rapid impact Perturbation, 'TRiP') is developed and its design requirements are tested in Chapter 7 . The TRiP includes a treadmill fitted with two specially designed breaking systems. An extending tripping leash is used to block one of the legs during the swing phase of gait to elicit a perturbation in gait. Controlled perturbations of varying characteristics (perturbation duration, obstruction timing and perturbation force) are induced with moderate to good accuracy. Perturbations of different forces can be simulated by manually adjusting the pressure on the brake, producing higher perturbation forces with increasing braking pressure. The duration of perturbation is reproduced within $12 \%$ accuracy of the set-values, showing high accuracy for perturbations $<150 \mathrm{~ms}$ and lower accuracy for perturbations with a duration of $200 \mathrm{~ms}$. The timing of the obstruction differs on average $15 \%$ from the set-values. A consistent time delay is observed between the synchronisation pulse and the actual occurrence of the perturbation (avg. 25ms), which partly explains the deviation in obstruction timing. In addition, 
two stereotypical recovery strategies (elevating and lowering) are elicited by the TRiP set-up. This study shows that the TRiP can be used to systematically explore the limits of walking stability. In Chapter $\mathbf{8}$ the TRiP set-up is used to investigate associations between age, walking speed, muscle strength, speed of information processing and the recovery response that individuals employ when they encounter a perturbation in gait. A large group of healthy young and older subjects are subjected to several perturbations in gait of varying characteristics. Older subjects who react more slowly, who are weaker and who walk slower employ more frequently the secure lowering strategy to recover, while the stronger, fast walking and faster reacting young subjects more often employ the more demanding elevating strategy. This suggests a shift from elevating strategy to lowering strategy with increasing age-related physiological changes. These findings correspond to previous studies showing clear effects of physiological factors (e.g. muscle strength) on walking stability and (in)adequate recovery responses. However, in contrast to the expectations based on previous studies, only weak associations $\left(R^{2}=0.23\right)$ are observed between the recovery response (proportion perturbations recovered by elevating strategy), walking speed, speed of information processing and muscle strength. In addition no direct effect of age is found. This suggests that the recovery response after being perturbed by the TRiP set-up is modified by a combination of multiple factors. In addition, it seems that individuals take account of the situation in which they are perturbed (e.g. tripping leash, obstacle, treadmill). More research is needed to investigate these associations further, to examine the remaining modifying factors (e.g. balance, coordination) and to investigate the effect of external factors (e.g. perturbation situation, environment) on the recovery responses.

In conclusion, acceleration-based gait analysis is reliable and sensitive to subject characteristics and health-related issues (e.g. functional knee limitations and fall risk). This, together with the practical benefits of AGA, shows that AGA is suitable for clinical use. The implementation of AGA in routine clinical practice, e.g. to diagnose pathological symptoms, to monitor a patient's function over time or to screen subjects at risk of falling, will provide an objective and detailed assessment of an individual's gait pattern. This demonstrates the additional value of AGA for clinical use. The gait database of healthy subjects serves as a reference.

The developed and validated TRiP set-up allows the investigation of stability during walking and has so far shown that the recovery response employed when gait is perturbed by the TRiP set-up is modified by a combination of multiple factors including e.g. muscle strength, speed of information processing and walking speed. 



\section{Samenvatting}


Lopen is de meest basale manier van voortbeweging voor mensen en is bovendien geassocieerd met welzijn, gezondheid en kwaliteit van leven. Het analyseren van het looppatroon van mensen is daarom klinisch relevant en wereldwijd geaccepteerd als een maat om de functie van individuen te bepalen. Gangbeeld analyse met behulp van geavanceerde bewegingsanalyse systemen is nauwkeurig, maar niet praktisch voor klinisch gebruik. Loopanalyse met behulp van klinische vragenlijsten is gemakkelijk en snel uit te voeren, maar heeft een beperkt onderscheidend vermogen. Dit benadrukt de vraag vanuit de klinische praktijk naar meer objectieve en precieze methoden om de functie van mensen te evalueren. Bewegingsanalyse met behulp van versnellingsmeters overbrugt de beperkingen van laboratorium-gebaseerde bewegingsanalyse systemen en klinische vragenlijsten. De veelbelovende resultaten van diverse versnellingsmeter configuraties om het looppatroon van mensen te analyseren en de praktische voordelen van een enkele drie-assige versnellingsmeter, suggereert dat een drieassige versnellingsmeter die versnellingen ter hoogte van het sacrum meet ('acceleration-based gait analysis', AGA) geschikt is als gangbeeld analyse systeem voor klinisch gebruik. Echter voordat $A G A$ in de klinische praktijk gebruikt kan worden, moet de toepassing van $A G A$ geëvalueerd worden in verschillende populaties en in verschillende settings. Dit is nog nooit eerder gedaan. Bovendien vereist dit een referentie database van gezonde personen. Dit proefschrift evalueert of een versnellingsgebaseerd gangbeeld analyse systeem bestaande uit een enkele drie-assige versnellingsmeter die versnellingen ter hoogte van het sacrum meet ( $A G A)$, geschikt is om het looppatroon van verschillende populaties te evalueren zoals van gezonde personen, patiënten met knie klachten en ouderen met een verhoogd valrisico. Bovendien wordt valrisico nader onderzocht omdat het analyseren van het looppatroon geen inzicht geeft in de oorzakelijke factoren van een val. Dit is echter wel nodig is om te begrijpen waarom mensen vallen en waarom ze een verhoogd valrisico hebben. Het laatste deel van dit proefschrift richt zich op verstoringen die kunnen ontstaan tijdens het lopen.

Dit proefschrift focust op drie onderzoeksvragen: De eerste onderzoeksvraag 'Is acceleration-based gait analysis (AGA) in staat om een gezonde referentie database te creëren op een betrouwbare manier?' wordt in hoofdstuk 2 \& 3 geëvalueerd. De onderzoeksvraag 'Is acceleration-based gait analysis (AGA) geschikt om toegepast te worden in de klinische praktijk bij patiënten met functionele beperkingen aan de onderste extremiteiten?' wordt in hoofdstuk 4 \& 5 onderzocht. Tot slot wordt de derde onderzoeksvraag 'Zijn loopparameters gemeten met behulp van acceleration-based gait analysis (AGA) en de herstel respons gebruikt voor externe verstoringen in het looppatroon geassocieerd met valrisico?' geëvalueerd in hoofdstuk 6, 7 en 8. 
Het doel van hoofdstuk 2 is het creëren van een referentie $A G A$ database van gezonde personen. Bovendien wordt de betrouwbaarheid van AGA om het looppatroon van mensen te beschrijven onderzocht. Het looppatroon van een grote groep gezonde personen met variërende persoonskenmerken is daarvoor gemeten met $A G A$. Spatio-temporele loop parameters zoals de loop snelheid en stap frequentie worden betrouwbaar gemeten met $A G A$. Andere klinisch relevante loopparameters, zoals de verticale verplaatsing van het lichaamszwaartepunt, de stap tijd asymmetrie en de loop irregulariteit, worden met matig tot goede betrouwbaarheid bepaald door AGA. De spatio-temporele AGA-parameters komen goed overeen met die van gevalideerde gangbeeld analyse systemen. Uit deze resultaten blijkt dat $A G A$ geschikt is om het looppatroon van gezonde personen in kaart te brengen buiten laboratorium settings, zoals in de klinische praktijk. Bovendien laten deze bevindingen zien dat $A G A$ robuust en onbeïnvloedbaar is voor mogelijke verschillen in uitvoering tussen waarnemers. Deze eigenschap is van groot belang voor het klinisch gebruik van $A G A$, waar verschillende waarnemers de functie test op verschillende locaties zullen uitvoeren. Een referentie database voor $A G A$-parameters van gezonde personen is gecreëerd, die als referentie dient. Deze referentie database laat toe om specifieke loopparameters te evalueren in vergelijking met een leeftijd, geslacht en lengte gematchte groep. Op deze manier kan het looppatroon van patiënten gemakkelijk en snel geëvalueerd worden. Bovendien dient het looppatroon correct geïnterpreteerd worden wat inzicht vereist in het effect van persoonskenmerken (bv. leeftijd, geslacht, lengte) op het looppatroon. Deze effecten worden in hoofdstuk 3 onderzocht en gekwantificeerd. Klinisch belangrijke relaties zijn gevonden tussen leeftijd, lengte, geslacht en AGAparameters. Deze persoonskenmerken verklaren $34 \%$ tot $51 \%$ van de variabiliteit in spatio-temporele loopparameters. Loop irregulariteit en stap tijd asymmetrie worden in mindere mate beïnvloed door persoonskenmerken (<7\%). Dit suggereert dat deze parameters meer waarschijnlijk gerelateerd zijn aan pathologische kenmerken. Typische relaties zijn gevonden tussen persoonskenmerken en loopparameters zoals een tragere loopsnelheid in vrouwen en ouderen ten opzichte van mannen en jongeren. De resultaten van deze studie laten zien dat het looppatroon variabel is tussen personen en benadrukken het belang van een gezonde referentie database. Uit hoofdstuk 2 en 3 kan geconcludeerd worden dat AGA bruikbaar is om het looppatroon van gezonde mensen op een betrouwbare manier te beschrijven. De database kan als referentie dienen voor klinische evaluaties.

In hoofdstuk 4 wordt onderzocht of $A G A$ bruikbaar is om onderscheid te maken tussen verschillende loop condities. Het looppatroon van gezonde personen die met 4 verschillende functionele knie beperkingen lopen wordt daarvoor geanalyseerd met behulp van AGA. Er is aangetoond dat AGA gebruikt kan worden om de verschillende loopcondities van elkaar te onderscheiden. Acute (kleine) 
veranderingen in het looppatroon zijn betrouwbaar gedetecteerd. De veranderingen in het looppatroon nemen in grootte toe naarmate de ernst van de gesimuleerde beperking. Slechts de loop irregulariteit is met minder grote betrouwbaarheid bepaald. Dit zou kunnen duiden op mogelijke variatie in loop irregulariteit gemeten tijdens de verschillende trials. Of het zou kunnen duiden op het feit dat $A G A$ minder bruikbaar is om de loop irregulariteit betrouwbaar te bepalen (bvb. door systematische fouten, beperkingen van de versnellingsmeter). De gevonden veranderingen in loopparameters zijn vergelijkbaar met vorige studies die gebruik maken van laboratorium gebaseerde gangbeeld analyse systemen. Een eerste klinische validatie studie is uitgevoerd in hoofdstuk 5, waar het gebruik van $A G A$ om het functioneren van orthopedische patiënten met symptomatische gonarthrose die op de wachtlijst staan voor een totale knie vervangende operatie, geëvalueerd is. AGA is hierbij gebruikt om het looppatroon van patiënten voor en na de operatie te analyseren. Bovendien is het looppatroon van patiënten vergeleken met het looppatroon van gezonde personen en zijn AGAparameters vergeleken met de hedendaagse klinische vragenlijsten. Uit deze studie blijkt dat de spatio-temporele loopparameters een goed onderscheidend vermogen hebben om het looppatroon van mensen met gonarthrose aan de knie te onderscheiden van het looppatroon van gezonde mensen. De loopsnelheid heeft hierbij het grootste onderscheidend vermogen. Bovendien is $A G A$ sensitief voor veranderingen in het looppatroon die gebeuren tijdens het herstel na een totale knie vervangende operatie. Ook liet $A G A$ een trager functioneel herstel zien vergeleken met de klinische vragenlijsten en zijn er geen of slechts zwakke correlaties gevonden tussen AGA-parameters en de klinische vragenlijsten. Dit toont aan dat $A G A$ extra informatie geeft over het functioneren van de patiënt. Hoofdstuk 4 en 5 tonen aan dat $A G A$ sensitief is voor verschillende functionele knie beperkingen (bv. gesimuleerde knie beperkingen en osteoartritis) en dat AGA in staat is om kleine, niet zichtbare veranderingen in het looppatroon te identificeren. Hieruit wordt geconcludeerd dat $A G A$ geschikt is voor klinische toepassingen. In de klinische praktijk kan $A G A$ gebruikt worden voor verschillende doeleinden, zoals om symptomen te identificeren, om een interventie te evalueren en om de functie van patiënten in de tijd te evalueren.

Hoofdstuk 6 evalueert associaties tussen AGA-parameters en de Tinetti vragenlijst, een maat om valrisico te beoordelen. Het looppatroon van ouderen is hiervoor geëvalueerd met behulp van $A G A$ en de Tinetti vragenlijst is afgenomen. Verschillen in AGA-parameters zijn gevonden tussen ouderen met verschillende Tinetti scores. Alle AGA-parameters correleren met de Tinetti schaal, maar slechts sterke correlaties zijn gevonden voor de loop snelheid, stap lengte en de root mean square van de verticale versnelling. Deze parameters hebben bovendien een root onderscheidend vermogen om ouderen te classificeren volgens de Tinetti schaal. Deze studie laat zien dat $A G A$ sensitief is voor valrisico gerelateerde loop 
parameters en bevestigt dat $A G A$ bruikbaar is om aspecten van val risico te meten. Echter zijn enkel zwakke tot matige correlaties gevonden tussen de Tinetti vragenlijst en de harmonic ratio en variabiliteitgerelateerde parameters, die in andere studies geassocieerd zijn met valrisico. Dit toont aan dat subjectieve meetinstrumenten om valrisico te beoordelen niet alle subtiliteiten in het looppatroon waarnemen. Dit benadrukt het belang om een objectieve gangbeeld analyse methode mee te nemen in de screening voor valrisico.

Hoofdstuk 7 en 8 onderzoeken de oorzaken van een val gedetailleerder en richten zich op verstoringen in het looppatroon. Om de stabiliteit tijdens lopen en de reactie op een verstoring tijdens lopen te onderzoeken is een laboratorium afhankelijke struikel opstelling ontwikkeld ("Timed Rapid impact Perturbation", 'TRiP'), die beschreven en getest is in hoofdstuk 7. De TRiP bestaat uit een loopband die uitgerust is met twee speciaal ontwikkelde rem systemen. Een rekbaar koord is gebruikt om één van de benen te verstoren tijdens de zwaai fase van het lopen, om op deze manier een verstoring in het looppatroon uit te lokken. Gecontroleerde verstoringen van verschillende omvang (duur, kracht en obstructie tijd) zijn teweeggebracht door de TRiP met matige tot goede nauwkeurigheid. Verstoringen van verschillende kracht zijn gesimuleerd door de druk op de rem manueel aan te passen. De verstoringskrachten nemen toe met toenemende remdruk. De duur van de verstoringen zijn gereproduceerd met minder dan $12 \%$ afwijking van de ingestelde duur. Verstoringen met een duur $<150 \mathrm{~ms}$ zijn met hoge nauwkeurigheid gereproduceerd, terwijl grotere afwijkingen gevonden zijn voor verstoringen met een duur van $200 \mathrm{~ms}$. De timing dat de verstoring plaats vindt in de loopcyclus verschilt gemiddeld $15 \%$ met de ingestelde timing. De consistente tijdsvertraging (gemiddelde $25 \mathrm{~ms}$ ) die gevonden is tussen de synchronisatie puls en het actueel plaatsvinden van de struikeling verklaart deze afwijking in timing gedeeltelijk. Bovendien zijn de twee stereotype herstel strategieën die in de literatuur beschreven staan, uitgelokt door de TRiP (elevating en lowering strategie). Deze studie toont aan dat de TRiP gebruikt kan worden om de stabiliteit tijdens lopen bij individuen te onderzoeken. Als gevolg daarvan is de TRiP toegepast in hoofdstuk $\mathbf{8}$ om associaties te onderzoeken tussen leeftijd, loop snelheid, spierkracht en reactietijd. Een grote groep gezonde jongeren en ouderen zijn daarvoor onderworpen aan verscheidene verstoringen van verschillend omvang. Oudere personen die trager lopen, die zwakker zijn en die trager reageren, herstellen vaker met de meest veilige lowering strategie, terwijl de elevating strategie vaker gebruikt wordt door de sneller lopende, sterkere en sneller reagerende jongeren. Dit suggereert dat er een verschuiving van elevating naar lowering strategie te zien is met leeftijdsgerelateerde fysiologische veranderingen. Dit suggereert ook dat valrisico, fysiologische veranderingen en de de herstel respons (proportie verstoringen herstelt met een elevating strategie) met elkaar associëren. Deze bevindingen komen overeen met vorige studies die 
een duidelijk effect van fysiologische factoren (bv. spierkracht) op de stabiliteit van lopen en een (in)adequate herstel respons vonden. Echter in tegenstelling tot de verwachting die gebaseerd is op vorige studies, worden slechts zwakke associaties $\left(R^{2}\right.$ 0.23) gevonden tussen de herstel respons en loop snelheid, reactietijd en spierkracht. Dit suggereert dat de herstel respons op verstoringen in het lopen, die teweeggebracht zijn door de TRiP set-up, beïnvloed wordt door een combinatie van verscheidene factoren. Bovendien suggereert het dat individuen zich aanpassen aan de situatie waarin ze verstoord worden (bv. voorwerp, loopband). Nader onderzoek is nodig om deze associaties nader te bestuderen en om het effect van andere interne (bv. coördinatie) en externe (bv. omgeving) factoren te onderzoeken.

Het onderzoek in dit proefschrift toont aan dat versnellinggebaseerd gangbeeld analyse (acceleration-based gait analysis, AGA) betrouwbaar en sensitief is voor persoonskenmerken (bv. leeftijd, geslacht) en gezondheidsaspecten (bvb. functionele knie beperkingen en valrisico). Deze resultaten, samen met de praktische voordelen van $A G A$, laten zien dat $A G A$ geschikt is voor klinisch gebruik. De implementatie van $A G A$ in de klinische praktijk om bijvoorbeeld een afwijkend looppatroon te identificeren, om het functioneren van patiënten te evalueren of om personen te screenen voor valrisico, zorgt voor een objectieve en gedetailleerde functie beoordeling. Dit benadrukt de toegevoegde waarde van AGA voor de klinische praktijk. De gecreëerde loop database voor gezonde personen dient dan als referentie.

De ontwikkelde en gevalideerde struikel set-up (Timed Rapid impact Perturbation, TRiP) kan gebruikt worden om de stabiliteit tijdens lopen nader te onderzoeken. Tot op heden is aangetoond dat de kwaliteit van de herstel respons na een verstoring in het lopen teweeggebracht door de TRiP, zwak geassocieerd is met loop snelheid, cognitie en spierkracht. Dit geeft aan dat meerdere factoren van invloed zijn op de herstel respons wanneer het lopen verstoord wordt door de TRiP set-up. 
Dankwoord 
Het mag dan wel 'mijn boekje' zijn, maar zonder de hulp, ideeën en interesse van velen was het nooit zo ver gekomen. Ik wil dan ook graag al deze mensen, met een aantal in het bijzonder, bedanken!

Mijn promotor en copromotoren: Ide, Kenneth, Bernd, en Hans. Bedankt voor jullie begeleiding, hulp en de open discussies van afgelopen jaren. Ook al was het niet altijd makkelijk om jullie ideeën te matchen, toch waren onze discussies vruchtbaar en leverde het steeds leuke ideeën op. Bedankt voor alles!

De leden van de beoordelingscommissie, Prof dr M.K.C. Hesselink, Prof. Dr.R. de Bie, Prof. Dr. R.J.E.M. Smeets, Prof. dr. A. Blom en Dr. M. Pijnappels voor het beoordelen van mijn thesis.

Alle vrijwilligers die bereid waren deel te nemen aan de diverse studies en de medewerkers van de valpoli Atrium MC Kerkrade en de verzorgingshuizen Scharweyerveld, Sint Gerlachus en de Zorgboog. Zonder jullie medewerking zou het uiteraard onmogelijk zijn geweest om dit proefschrift af te ronden. Dankjewel! Alle collega's van de Universiteit Maastricht, de afdeling Orthopaedie van het Atrium MC Heerlen en de Maatschap Orthopaedie, bedankt!

Maar met name dankjewel m'n vaste uni-kamergenootjes en paranimfen Herman en Janneke en aan Tom. Bedankt voor de leuke werksfeer, de gezellige koffiepauzes, de onnoemlijke discussies, zowel over studies als over andere erg interessante anti-research onderwerpen. Ons tripje naar Kaapstad zal me altijd bijblijven! Paul, bedankt voor de technische ondersteuning, het programmeren in matlab en je inzet voor de TRiP. Harry bedankt voor de technische hulp die ik geregeld nodig had met $\mathrm{m}$ 'n laptopje en Jos bedankt voor je hulp en interesse in m'n studies. Mn allerliefst kamergenootje in Heerlen, Wendy. Jouw hulp als vertaalster, jouw ideeën, jouw luisterend oor, de fijne gesprekken en de gezellige cappucino-momenten hebben me geholpen om dit uiteindelijk te bereiken. Ik hoop dat we nog vele jaartjes ons kamertje mogen delen in Heerlen. Wouter, bij jou begon het allemaal. Mede dankzij jou ligt hier nu mijn boekje. Bedankt voor je vertrouwen, je interesse in dit boekje en de (vele) duwtjes in mn rug!

Alle studenten die hebben bijgedragen. Bedankt voor jullie hulp met het includeren van proefpersonen, het verrichten van de metingen, het brainstormen en meedenken. Ik wens jullie allen veel succes toe in de toekomst.

En tot slot, lieve Bernie, pap, mam, llona, Ben en Bertha, zoals jullie ondertussen wel weten gaat het schrijven van een proefschrift niet zonder slag of stoot. Bedankt dat ik bij jullie mijn hart kon luchten, dat jullie er altijd voor me waren, niet alleen 
voor de strijk of een lekker etentje ;), maar ook voor een gezellige babbel. Loontje, ik ben zo blij dat jij mijn grote zusje bent. Pap en mam, bedankt dat jullie me zoveel kansen gegeven hebben. Dankzij jullie werd ik kampioen met dansen, en dankzij jullie is me nu ook dit gelukt. Ik weet nu dat je veel kan bereiken, als je er maar voor gaat! Ber, jij bent al meer dan 11 jaar mijn allergrootste maatje. Samen hebben we al heel wat meegemaakt, onder andere de verbouwing van ons kasteeltje, de vele dans kampioenschappen, maar ook mijn promotietraject, wat niet altijd makkelijk (ik bedoel gezellig) was. Ber, mercie vuer alles, ich bin é zoe bliej vuer éédere daag bie dich heem te koeme.

Dankjewel allemaal! 



\section{List of publications}




\section{Publications}

Senden R, Grimm B, Heyligers IC, Savelberg HHCM, Meijer K. An acceleration-based gait test for healthy subjects: reliability and reference data. Gait Posture 2009;30(2):192-196

Senden R, Grimm B, Meijer K, Savelberg H, Heyligers IC. The importance to including objective functional outcomes in the clinical follow up of total knee arthroplasty patients. The Knee, 2010; 18 (5):306311.

Senden R, Heyligers IC, Meijer K, Savelberg HHCM, Grimm B. Acceleration-based motion analysis as a tool for rehabilitation: Exploration in simulated functional knee limited walking conditions. Am J Phys Med \& Rehabil 2011; 90(3): 226-232

Senden R, Meijer K, Heyligers IC, Savelberg HHCM, Grimm B. The importance of correcting for individual differences in the clinical diagnosis of gait disorders. Physiotherapy 2012;98:325-329

Senden R, Savelberg HHCM, Grimm B, Heyligers IC, Meijer K. Accelerometry-based gait analysis, an additional objective approach to screen subjects at risk for falling. Gait Posture, 2012; 26(2):296300.

van Hemert WL, Senden R, Grimm B, van der Linde MJ, Lataster A, Heyligers IC. Early functional outcome after subvastus or parapatellar approach in knee arthroplasty is comparable. Knee Surg Sports Traumatol Arthrosc. 2010 Oct 17.

van Hemert WL, Senden R, Grimm B, Kester AD, van der Linde MJ, Heyligers IC.Patella retention versus replacement in total knee arthroplasty; functional and clinometric aspects. Arch Orthop Trauma Surg. 2009 Feb;129(2):259-65. Epub 2008 May 17.

Karel JM, Senden R, Janssen JE, Savelberg HM, Grimm B, Heyligers IC, Peeters R, Meijer K. Towards unobtrusive in vivo monitoring of patients prone to falling. Conf Proc IEEE Eng Med Biol Soc. 2010;1:5018-21.

Senden R, Savelberg HHCM, Willems P, Grimm B, Heyligers IC, Meijer K. The 'TRiP', a specially designed experimental set-up to induce perturbations in a standardised way: a technical note. Submitted for publication.

Senden R, Savelberg HHCM, Adam JJ, Grimm B, Heyligers IC, Meijer K. The influence of age, muscle strength and speed of information processing on recovery response to external perturbations in gait. Submitted for publication.

\section{Proceedings (Orals and Posters)}

Van Hemert WLW; Senden R; Grimm B; Heyligers IC. Gait analysis on knee arthroplasty patients with and without patella resurfacing. Orthopaedic Research Society, ORS 2006, Chicago, USA, (poster).

Grimm B, Senden R, Vanderhenst T, Munch C, Meijer K, Heyligers IC. Clinically relevant output parameters of an accelerometer based movement analysis system. Joint ESMAC - GCMA (JEGM) 2006, Amsterdam (poster).

Senden R, Meijer K, Heyligers I, Grimm B. Characterizing pathological gait in men and women using a triaxial accelerometer. Combined meeting of the Orthopaedic Research Societies, CORS 2007, Honolulu, Hawai (oral).

Senden R, G. Geurts, Heyligers IC, Meijer K, Grimm B. An inertial measurement system that differentiates between healthy and pathological gait. International Society of Biomechanics, ISB 2007, Taipei, Thaiwan, (oral).

Van Hemert WLW, Senden R, Grimm B, Heyligers IC. Gait analysis on knee arthroplasty patients with and without patella resurfacing. Orthopaedic Research Society, ORS 2007, San Diego, USA. (oral).

Van Hemert WLW, Grimm B, Senden R, Heyligers IC. Resurfacing or retaining the patella in total knee arthroplasty: A movement analysis study. European Federation of Orthopaedics and Traumatology, EFORT 2007, Florence, Italy, (oral).

Senden R, Geurts G, Heyligers IC, Meijer K, Grimm B. Sensitivity and reliability of an acceleration based motion system. Int. Biomechanics Conference 2007, Salford, UK, (oral).

Senden R, Geurts G, Heyligers IC, Meijer K, Grimm B. Analysis of pathological gait using accelerometry. European Society of Movement of Adults and Children, ESMAC 2007, Athens, Greece, (poster). 
Senden R, Geurts G, Meijer K, Heyligers IC, GrimmB. Sensitivity of an inertial motion analyse tool for clinical gait evaluation. European Workshop on Movement Sciences, EWOMS 2007, Amsterdam, (oral).

Senden R, Grimm B, Heyligers IC. Measuring gait differences between total knee arthroplasty patients and healthy subjects using accelerometers. Orthopaedic Reseasch Society, ORS 2008, San Francisco, USA, (poster).

Senden R, Grimm B, Van Hemert W, Meijer K, Savelberg H, Heyligers IC. Validation of an acceleration based gait test. European Federation of National Associations of Orthopaedics and Traumatology, EFORT 2008, Nice, France, (oral).

Senden R, Grimm B, Meijer K, Heyligers IC. Motion analysis with accelerometers for routine clinical follow-up: A promising technique? European Federation of National Associations of Orthopaedics and Traumatology, EFORT 2008, Nice, France, (eposter).

Senden R, Heyligers IC, Meijer K, Hemert WLW van, Grimm B. Acceleration based gait test to monitor patients after total knee surgery. European Orthopaedic Research Society, ORS 2008, Milan, Spain, (oral).

Verlaan L, Senden R, Storken G, Heyligers IC, Grimm B. Outcome assessment in orthopaedics using a stair climbing test and accelerometer based motion analysis: a feasibility study. European Orthopaedic Research Society, EORS 2008, Milan, Spain, (oral).

Senden R, Grimm B, Meijer K, Heyligers IC. Reference gait parameters from a functional test suitable for routine clinical follow up. Nordic Orthopaedic Federation, NOF 2008, Amserdam, (oral).

Senden R, Grimm B, Meijer K, Heyligers IC. A new functional test to complement classic outcome scores in knee arthroplasty. Nordic Orthopaedic Federation, NOF 2008, Amserdam (oral).

Verlaan L Grimm B, Senden R, Storken G, Heyligers IC. A stair climbing test to score clinical outcome in orthopaedics. Nordic Orthopaedic Federation, NOF 2008, Amserdam (poster).

Senden R, Grimm B, Meijer K, Heyligers IC. 3D Analysis of Human Movement of Int Soc Biomech, 3DMA 2008, Amsterdam, (poster).

Grimm B, Senden R, Meijer K, Heyligers IC. Evaluating inertia based gait analysis for routine clinical outcome assessment. American Society of Mechanical Engineers, ASME 2008, Boston, USA, (oral)

Senden R, Grimm B, Verlaan L, Meijer K, Heyligers IC. Inertia based motion analysis of stair climbing: Demographic differences and validatity in knee arthroscopy. Symposium Bath 2008

Senden R, Heyligers IC, Meijer K, Savelberg H, Grimm B. Validation of an acceleration based gait test to follow up TKA patients. Int Conf Ambulatory MOnitoring of Physcial Activity \& Movement, ICAMPAM 2008, Rotterdam, (poster).

Verlaan L, Senden R, Heyligers IC, Grimm B. Orthopaedic outcome assessment with accelerometer assessed stair climbing. Int Conf Ambulatory MOnitoring of Physcial Activity \& Movement, ICAMPAM 2008, Rotterdam, (poster).

Senden R, Melai T, Savelberg HHCM, Grimm B, Heyligers IC, Meijer K. Detecting Near falls from accelerations during perturbed treadmill walking. International Society of Biomechanics, ISB 2009, CapeTown, South Africa. (oral).

Senden R, Grimm B, Heyligers IC, Melai T, Savelberg HHCM, Meijer K. Detecting Near Falls from accelerations during perturbed treadmill walking. Symposium Bath 2009.

Senden R, Grimm B, Meijer K, Savelberg HHCM, Heyligers IC. An acceleration based gait test to monitor patients over time. Bio-Medical Engineering Conference, BME 2009 Egmond aan Zee, (oral).

Meijer K, Senden R, Arnoldussen E, Grimm B, Heyligers IC, Kenney L, Preece S, Savelberg H. A real time monitor for falls and stumbles during activities of daily life. Bio-Medical Engineering Conference, BME 2009 Egmond aan Zee, (oral).

Senden R, Grimm B, Verlaan L, Meijer K, Savelberg HHCM, Heyligers IC. Validity of an acceleration based stair test for clinical use. Bio-Medical Engineering Conference, BME 2009 Egmond aan Zee, (poster).

Senden R, Grimm B, Meijer K, Heyligers IC. The relevance of scaling in clinical gait analysis. European Federation of National Associations of Orthopaedics and Traumatology, EFORT 2009, Vienna, (poster).

Grimm B, Heyligers I, Senden R, Storken G, Verlaan L. Validation of a sit-stand-sit test as an orthopaedic outcome measure using inertia based motion analysis. European Federation of Orthopaedics and Traumatology, EFORT 2009, Vienna, (oral). 
Verlaan L, Grimm B, Senden R, Heyligers IC. Inertia based motion analysis to support the diagnosis of meniscal tears in patients indicated for knee arthroscopy. European Federation of National Associations of Orthopaedics and Traumatology, EFORT 2009, Vienna, (oral).

Senden R, Can an Accelerometer based Gait Test assess Fall Risk? A Comparison with the Tinetti score. American Association of Orthopaedic Surgery, AAOS 2010 New Orleans, USA. (poster).

Senden R, Grimm B, Heyligers IC, Savelberg HHCM, Willems P, Meijer K.. Fall risk assessment using an acceleration based gait test. European Federation of Orthopaedics and Traumatology, EFORT 2010, Madrid, Spain, (poster).

Meijer K, Senden R, Laeven P, Savelberg HHCM. The Trip, a device for timed rapid induced perturbation during treadmill walking. National Congress of Theoretical and Applied Mechanics. Pennsylvania, 2010, (oral)

Senden R, Meijer K, Heyligers IC, Savelberg H, Tilburgh K, Schoeberichts S, Willems P, Grimm B. Which is the best way to analyze an acceleration-based gait test for routine clinical follow up? European Orthopaedic Research Society, EORS 2010, Davos, (oral).

Senden R, Savelberg HHCM, Adam JJ, Grimm B, Heyligers IC, Meijer K. Differences in trip recovery between young and elderly subjects. European Orthopaedic Research Society, EORS 2010, Davos, (poster).

Senden R, Savelberg HHCM, Adam JJ, Grimm B, Heyligers IC, Meijer K. The influence of muscle strength and speed of information processing on recovery strategy after stumbling. World Congres on Biomechanics, WCB 2010, Singapore, (oral).

Lipperts M, Senden R, Van Asten W, Heyligers IC, Grimm, B. Validation of a low cost activity monitor for orthopaedic patients. European Federation of Orthopaedics and Traumatology, EFORT 2011, Copenhagen, Denmark, (oral).

Grimm B, Senden R, Meijers W, Van Der Linde M, Heyligers IC. Prospective 5-8 years follow-up of the Scorpio TKR system, a single radius design. European Federation of Orthopaedics and Traumatology, EFORT 2011, Copenhagen, Denmark, (poster).

Senden R, Savelberg HHCM, Adam J, Grimm B, Heyligers IC, Meijer K. Is there a role for cognitive therapy in fall prevention? European Federation of Orthopaedics and Traumatology, EFORT 2011, Copenhagen, Denmark, (posters).

Senden R, Willems P, Savelberg H, Grimm B, Heyligers IC, Meijer K. Validation of the TRiP, a device for timed rapid induced pertruabtions during treadmill walking. International Society of Biomechanics, ISB 2011, Brussels, (oral).

Lipperts M, van Laarhoven S, Senden R, van Asten W, Heyligers IC, Grimm B. Acclerometer based activity monitoring of patient-like stair climbing with crutches and "step by step"technique. International Society of Biomechanics, ISB 2011, Brussels, (oral).

Senden R, Bolink S, Heyligers IC, Meijer K, Savelberg H, Grimm B. How to derive gait parameters from an acceleration-based gait test? International Society of Biomechanics, ISB 2011, Brussels, (poster).

Vles GF, Senden R, Heyligers IC, Samijo S, Grimm B. Inertia-based flapping and clapping test: a promising tool for function assessment in patients with shoulder complaints. SICOT 2011, Prague, (oral).

Senden R, Heyligers IC, Grimm B. Psychological factors affect the postoperative gait performance of total hip arthroplasty patients. SICOT 2011, Prague, (poster).

Van der Lingen J, Senden R, Heyligers IC, Hemert van W, Grimm B. Inertia sensor based motion analysis of the spine: A clinical feasibility study in patients with nonspecific low back pain. European Orthopaedic Research Society, EORS 2011, Vienna, (oral).

Senden R, Heyligers IC, Coenen P, Grimm B. Challenging the reliability of the knee society score. European Orthopaedic Research Society, EORS 2011, Vienna, (oral).

Van Laarhoven S, Lipperts M, Liu WY, Senden R, van Asten W, Heyligers IC, Grimm B. Validation of an acitivity monitor for total hip or knee arthroplasty patients. European Orthopaedic Research Society, EORS 2011, Vienna, (oral).

Senden R, Coenen P, Heyligers IC, Grimm B. Evaluating Different Functional Outcome Measures: Clinician-Administered Scales vs. Patient-Administered Outcome Measures vs. Objective Functional Tests. International Society for Technology in Arthroplasty, ISTA 2011, Bruges, Belgium, (oral).

Van Laarhoven S, Lipperts M, Senden R, van Asten W, Heyligers IC, Grimm B. Accelerometry based monitoring of in-patient activity to objectively evaluate TJA rehabilitations programs. International Society for Technology in Arthroplasty, ISTA 2011, Bruges, Belgium, (oral). 
Lipperts M, Senden R, Schoeberichts S, Van Asten W, Heyligers IC, Grimm B. Activity monitoring in orthopaedics: Improving validation methods and detection accuracy. Orthopaedic Research Society, ORS 2011, Long Beach, USA, (poster).

Senden R, Bolink S, van laarhoven S, Heyligers IC, Grimm B. Inertia-sensor based motion analsyis as outcome measure for routine clinical application in patients with lower extremity problems. European Orthopaedic Society, EORS 2012, Berlin, (poster).

Senden R, Heyligers IC, Grimm B. Reporting clinical outcome scales: proportions can uncover critical information hidden in the mean values. European Federation of National Associations of Orthopaedics and Traumatology, EFORT 2012, Berlin, (oral).

Senden R, Heyligers IC, Grimm B. Poor reproducibility of the knee society scores challenges it's value for clinical follow up and comparative studies. European Federation of National Associations of Orthopaedics and Traumatology, EFORT 2012, Berlin, (oral).

Senden R, Heyligers IC, Renkens S, Grimm B. High variability in knee society score between studies: how reliable is the knee society score? American Association of Orthopaedic Surgery, AAOS 2012, San Francisco, USA, (poster).

Van Laarhoven S, Lipperts M, Senden R, van Asten W, Heyligers IC, Grimm B. Wearable Sensor for inpatient activity monitoring to improve arthroplasty recovery programs, Orthopaedic Research Society, ORS 2012, San Francisco, USA, (poster).

Senden R, Dinjens R, Heyligers IC, Grimm B. Evaluation of the new knee society score. Nordic Orthopaedic Federation, NOF 2012, Tallinn, (oral).

Senden R, Heyligers IC, Vencken W, Grimm B. Scoring satisfaction, is this a trustable outcome measure. Nordic Orthopaedic Federation, NOF 2012, Tallinn, (oral).

Senden R, Heyligers IC, Grimm B. The effect of shortening the walkway in clinical inertia-based gait analysis. European Orthopaedic Research Society, EORS 2012, Amsterdam, (oral).

Dinjens R, Senden R, Heyligers IC, Grimm B. A short form of the new KSS is a valid and practical clinical outcome tool for the younger and more demanding patient. European Orthopaedic Research Society, EORS 2012, Amsterdam, (Oral).

Senden R Linsen B, van Laarhoven S, Lipperts M, Heyligers IC, Grimm B. Physical activity monitoring in knee arthroplasty patients using a body-fixed sensor: reference data and comparison with clinical scores. European Orthopaedic Research Society, EORS 2012, Amsterdam, (oral).

Cox S, Boymans T, Senden R, Heyligers IC, Grimm B. Knee angle measurements on 3D CT are influenced by femur orientation, slice thickness and reslicing. European Orthopaedic Research Society, EORS 2012, Amsterdam, (oral).

Dinjens R, Senden R, Heyligers IC, Grimm B. The new knee society score: Evaluation of the clinimetric quality in Dutch patients. European Orthopaedic Research Society, EORS 2012, Amsterdam, (poster).

\section{INVITED SPEAKER:}

7th Meeting Combined Orthopaedic Research Society, CORS Kyoto Japan, 2010. 16-19 October. R. Senden. New Questionnaire based outcome scores for research and clinical follow-up. Invited speaker, Sunday, October 17, 2010, 7:30-9:00, Workshop 3 Room E

Chinese Orthopaedic Association, COA-IORS, Beijing, China, 2012, 15-18 November. R. Senden. Motion Analysis for routine clinical practice using ambulant sensors. Invited speaker, Friday, November 17, 2012, 11:00-12:15, Symposium VI, European ORS Symposium 'Outcome assessment in clinical practice and research: Challenges, new tools and seeking consensus'. 

About the Author 
Rachel Senden was born on January 22nd, 1983 in Tongeren, Belgium. In 2001 she completed secondary school at the 'Provinciale Secundaire School' $s$ ' Gravenvoeren. The same year, she started the study Health Sciences at the University Maastricht, where she graduated in 2005 as a Master in Health Sciences, specialization Movement Sciences. After graduating, Rachel worked for seven months as scientific researcher at the Department Movement Science at the Maastricht University and at the Department Orthopaedics and Traumatology at the Atrium MC Heerlen. In October 2006 she started her PhD project, titled "Ambulatory gait analysis: Clinical application and fall risk detection" which was approved in May 2013. She performed measurements at different locations such as the orthopaedic department of the Atrium MC Heerlen, the fall clinic at the Atrium MC Kerkrade, the movement lab of the Maastricht University and at several nursing homes located at Maastricht (Scharweyerveld and Sint Gerlachus). During her PhD project she presented several abstracts at national and international conferences. Rachel attended a workshop at the combined Orthopaedic Research Society in Kyoto, Japan, which was titled "New tools for routine clinical outcome assessment: from changing patient demands to improved questionnaires, motion analysis and activity monitor'. In addition she was an invited speaker at the Chinese Orthopaedic Association in Beijing, China, attending the European ORS Symposium titled "Motion Analysis for routine clinical practice using ambulant sensors." Since October 2010, Rachel started as scientific researcher and coordinator at the department of Orthopaedics and Traumatology of the Atrium MC in Heerlen, where she continues further research on human movement analysis.

Dancing has a dominant role in Rachel's life. She started dancing when she was 5 years old, trained many hours a week and won several national and international competitions. From the year 2000, she became successful coach in the European Federation for Dance sport Organizations (EFDO). 
Yesterday is a history

Tomorrow is future,

This moment is a gift

That's why it is called present 